*ak RMIS View/Frint Document Cover Sheet tow

This document was retrieved from the Documentation and Records Manaqement (DRM) ISEARCH System. It is intended for Information only and may not be the most recent or updated version. Contact a Document Service Center (see Hanford Info for locations) if you need additional retrieval information.

Accession \#: D196071642

Document \#: SD-WM-CN-016

Title/Desc:

XQS \& UNIT DOSE CALCULATIONS FOR CWC INTERIM SAFETY BASIS EFFORT

Pages: 124 

SN\&NE

\section{Proj./Prog./Dept./Div.:}

Solid Waste SAR/8M300

8. Originator Remarks:

The attached SD provides the information of $X / Q$ s and unit dose calculations for Central Waste Complex Interim Safety Basis Effort.
2. To: (Receiving Organization)
3. From: (Originating Organization) Consequence Analysis $8 M 400$

\section{Cog. Engr.:}

C.H. Huang

\section{Receiver Remarks:}

4. Related EDT No.:

N/A

7. Purchase Order No.:

$$
\mathrm{N} / \mathrm{A}
$$

9. Equip./Component No.: $N / A$

10. System/Bldg./Facility: CWC

12. Major Assm. Dwg. No.: $N / A$

13. Permit/Permit Application No.: $N / A$

14. Required Response Date:

$$
2 / 29 / 96
$$

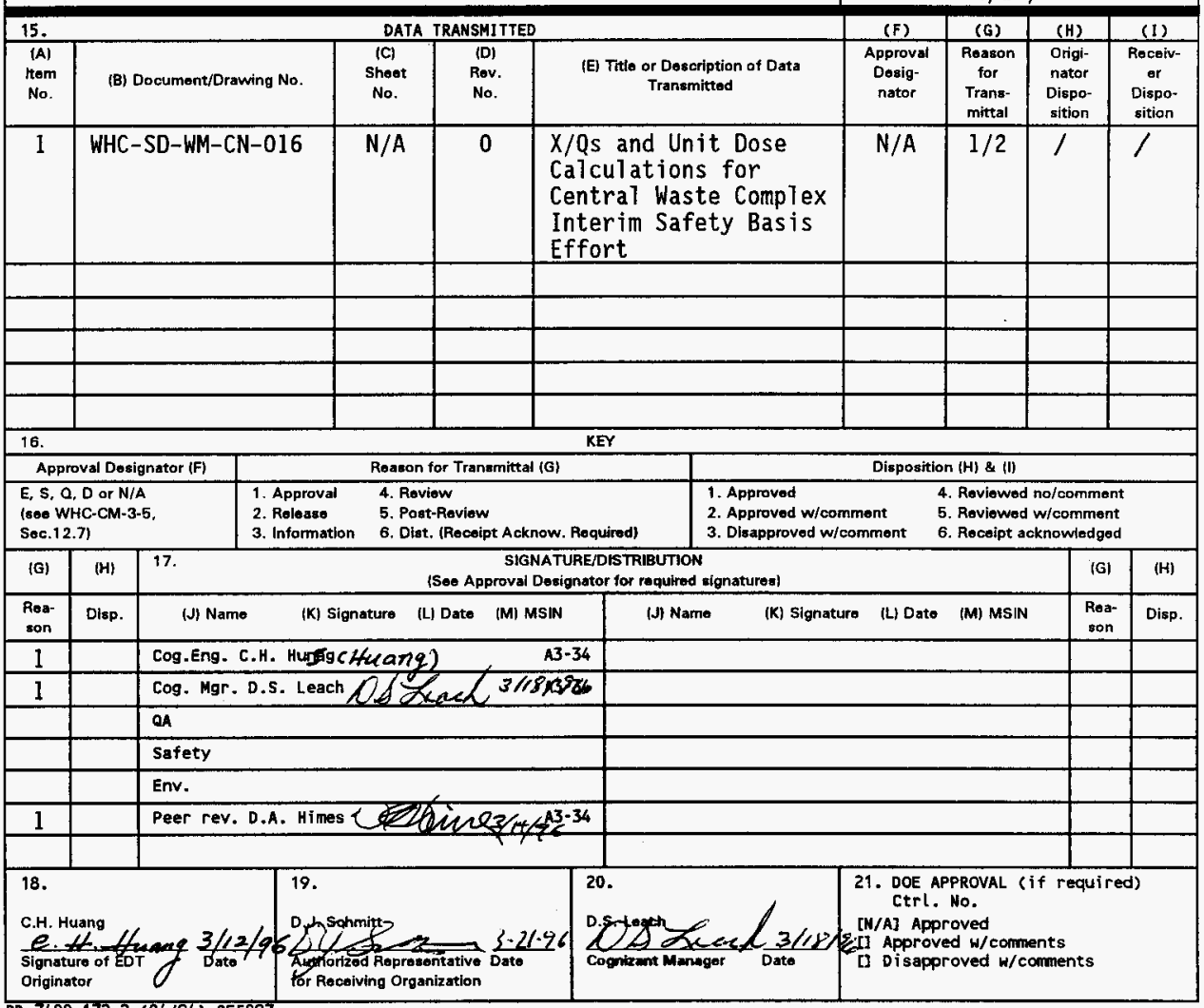




\title{
XQS AND UNIT DOSE CALCULATIONS FOR CENTRAL WASTE COMPLEX INTERIM SAFETY BASIS EFFORT
}

\author{
C. H. HUANG
}

Westinghouse Hanford, Richland, WA 99352

U.S. Department of Energy Contract DE-AC06-87RL10930

$\begin{array}{lll}\text { EDT/ECN: } & 156464 & \text { UC: } 630 \\ \text { Org Code: } & 8 M 400 & \text { Charge Code: A4G12 } \\ \text { B\&R Code: } & \text { EW3130020 } & \text { Total Pages: } 18 Q 121{ }_{4,36}, 96\end{array}$

Key Words: Atmospheric Dispersion, Dose Calculation, Central Waste Complex, Fire Accident, CaTculation Note.

Abstract: This document provides the values of $X / Q$ s for the onsite and offsite receptors, taking into account the building wake and the atmospheric stability effects. $X / Q$ s values for the potential fire accident were also calculated. In addition, the unit doses were calculated for the mixtures of isotopes.

TRADEMARK DISCLAIMER. Reference herein to any specific commercial product, process, or service by trade name, trademark, manufacturer, or otherwise, does not necessarily constitute or imply its endorsement, recommendation, or favoring by the United States Government or any agency thereof or $i$ ts contractors or subcontractors.

Printed in the United States of America. To obtain copies of this document, contact: WHC/BCS Document Control Services, P.0. Box 1970, Mailstop H6-08, Richland WA 99352, Phone (509) 372-2420; Fax (509) 376-4989.
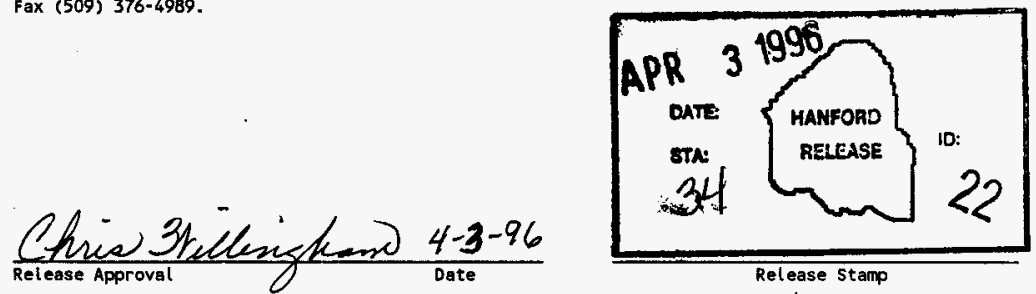

Approved for Public Release 
WHC-SD-WM-CN-016, Rev. 0

\title{
$X / Q S$ AND UNIT DOSE CALCULATIONS FOR CENTRAL WASTE COMPLEX INTERIM SAFETY BASIS EFFORT
}

\author{
C. H. HUANG
}

March 13, 1996

\section{INTRODUCTION:}

The objective for this problem is to calculate the ground-level release dispersion factors $(X / Q)$ and unit doses for onsite facility and offsite receptors at the site boundary and at Highway 240 for plume meander, building wake effect, plume rise, and the combined effect. The release location is at Central Waste Complex Building P4 in the 200 West Area. The onsite facility is located at Building P7.

Acute ground leve 1 release 99.5 percentile dispersion factors $(X / Q)$ were generated using the GXQ (B. Hey, 1994). The unit doses were calculated using the GENII code.

The dimensions of Building $\mathrm{P} 4$ are $15 \mathrm{~m}$ in $\mathrm{W} \times 24 \mathrm{~m}$ in $\mathrm{L} \times 6 \mathrm{~m}$ in $\mathrm{H}$.

\section{METHODOLOGIES AND ASSUMPTIONS}

Bounding or worst case meteorology is considered to be a set meteorological conditions (i.e., wind speed, stability class) which results in a downwind concentration of airborne contaminants which is exceeded only a small fraction of time due to weather fluctuations. The NRC (1982) defines this fraction of exceedance as $0.5 \%$ for each sector or $5 \%$ for the overall site. That is, $X / Q$ values are generated for weather conditions which result in downwind concentrations which are exceeded onty $0.5 \%$ of the time in the maximum sector, or $5 \%$ of the time for the overall site. The greater of these two values is selected as bounding. Bounding or worst case $X / Q$ values are used to assess the dose consequences in most accident scenarios. These bounding $X / Q$ values represent minimum dispersing conditions, which results in maximum downwind concentrations, i.e., concentrations which are exceeded only a very small fraction of the time, as explained above. These $X / Q$ 's will therefore result in very conservative estimates of potential accident consequences.

The $X / Q$ refers to the concentration of airborne contamination. It is the ratio of contaminant concentration at a specific receptor location to contaminant release rate. The receptor of concern is normally defined as a human having a standard breathing rate and metabolism. The product of $X / Q$ and breathing rate is a unitless number representing the fraction of contaminant inhaled to contaminant released. Inhalation is the most 1 ikely pathway to human exposure.

The GENII code was used to calculate the unit doses. 


\section{BUILDING WAKE EFFECT AND PLUME MEANDER}

The formulas used to calculate the horizontal diffusion and vertical diffusion coefficients at the source were taken from Equation 2.2 of NRC (1990) for considering the building wake effect:

$$
\begin{aligned}
& \sigma_{y}(x=0)=W_{b} / 4.3=15 / 4.3=3.5 \\
& \sigma_{z}(x=0)=H_{b} / 2.15=6 / 2.15=2.8
\end{aligned}
$$

where:

$$
\begin{aligned}
& W_{b}=\text { Building width }(m) \\
& H_{b}=\text { Building height }(m)
\end{aligned}
$$

The above adjustment for the calculation of $X / Q$ is made using the virtual distance method. These adjusted virtual distances are then used to calculate downwind diffusion coefficients and the concentrations.

The correction of plume meander used in the MACCS code (as requested by the customer) was used for adjusting the $X / Q$ determination.

\section{BUOYANT PLUME RISE}

The release scenario is applied to a postulated ground level fire. The plume rise is also considered in the dose calculations. The buoyancy of the plume depends upon the heat generated or the combustion gas temperature.

The momentum rise of the plume is small in comparison to the buoyant component. The buoyant plume rise is a function of gas density and velocity. The velocity is a function of fire size and geometry. The momentum/buoyancy plume rise model used in GXQ is taken from equations $8.35,8.36$, and 8.57 of Randerson (1984) for plume rise in a crosswind. Assumptions made are that the effluent is a heated gas with a heat capacity and mean molecular weight close to that of air, and that initial effluent density and vertical velocity within the plume are constant.

\section{FIRE SCENARIO ASSUMPTIONS}

An initial vertical velocity of $1 \mathrm{~m} / \mathrm{s}$ is assumed. This value is both conservative and consistent, assuming that the temperature of combustion gas is $1200^{\circ} \mathrm{F}$. The size of the fire is conservatively kept small at 3.0 meter diameter. The convective heat release rate for the diesel (modelled as kerosene) fire is $3.35 \times 10^{6} \mathrm{~W}$ based on a burn rate of $80.1 \mathrm{~g} / \mathrm{s}$ for a burn area of $7.07 \mathrm{~m}^{2}$ (see NRC, 1988; NUREG - 1320).

The duration of the fire is assumed to be half an hour. Wake effects and plume mender are also included in the $X / Q$ calculations. 
WHC-SD-WM-CN-016, Rev. 0

\section{RECEPTOR DESCRIPTIONS}

- Onsite: Ground level release dispersion factors $(X / Q)$ were generated using the GXQ dosimetry code for the onsite nearest occupied facility for various cases with plume meander, building wake effect or plume rise for acute exposure at Building P7. Building P7 is located $498 \mathrm{~m}$ in the direction of east-southeast (ESE) from Central Waste Complex Building P4. These results are summarized in the Table 2 for various cases.

- Site Boundary: Receptor at the site boundary in the worst direction. Where the site is bounded by the Columbia River, the site boundary is taken to be at the nearer bank of the river. Atmosphere dispersion factors $(X / Q)$ were generated for 16 sectors location around the site boundary; plume rise is also included in a fire accident for calculating the acute exposure. These results are summarized in the Table 3 .

- Receptor at Highway 240: In addition, the receptor at Highway 240 were calculated. Atmosphere dispersion factors $(X / Q)$ were generated for sectors location around the offsite receptor at Highway 240 with plume meander, building wake effect, plume rise, and the combined plume meander and building wake for acute exposure. These results are summarized in the Table 4.

The receptor locations and the source terms are specified by the customer (see Appendix A). The receptor locations at the onsite facility, the offsite boundary, and Highway 240 are given in Table 1 .

\section{CODE DOCUMENTATION}

o GXQ version $4.0(11 / 19 / 94)$

o Joint Frequency Data: 200 Area, $10 \mathrm{~m}$, Pasqui11 A-G (19831991 Average).

o GENII version $1.485(12 / 3 / 90)$

- GENII Default Parameter Values (28-Mar-90 RAP)

o Radionuclide Master Library (7/23/93 PDR)

- External Dose Factor Library (8-May-90-RAP)

- Internal Dose Increments, PNL or Maximum Solubilities, (7/23/93 PDR)

GXQ output files are attached in Appendix B. GENII input and output files are attached in Appendix $C$. 
WHC-SD-WM-CN-016, Rev. 0

Table 1. Receptors Locations in the Worst Sector for Onsite Facility, Offsite, and Highway 240.

\begin{tabular}{||c|c|}
\hline Receptor & Distance \\
\hline $\begin{array}{c}\text { Onsite Facility (P7) } \\
\text { (ESE) }\end{array}$ & $498 \mathrm{~m}$ \\
\hline Offsite (W) & $11.7 \mathrm{~km}$ \\
\hline Highway $240(W)^{*}$ & $4070 \mathrm{~m}$ \\
\hline
\end{tabular}

* West is the worst direction (see Case 4 GXQ run in Appendix $B$ for the directions checked).

Table 2: $\quad$ Onsite Nearest Occupied Facility 99.5 Percentile Dispersion Factors $(X / Q)$ at the Receptor of Building P7.

\begin{tabular}{|c|c|c|c|c|}
\hline spect f ic $\times 10$ & Buil lajng wake & Plume Meander & Plume Rise & $\mathrm{x} / \mathrm{o}\left(\mathrm{s} / \mathrm{m}^{3}\right)$ \\
\hline 1 & $Y$ & $Y$ & $Y$ & $3.13 E-05$ \\
\hline 2 & $Y$ & $Y$ & N & $1.36 \mathrm{E}-03$ \\
\hline 3 & $Y$ & N & N & $1.47 \mathrm{E}-03$ \\
\hline 4 & $n$ & N & $n$ & 2. $10 \mathrm{E}-03$ \\
\hline 5 & N & $y$ & $Y$ & $3.13 \mathrm{E}-05$ \\
\hline 6 & H & N & Y & $9.77 \mathrm{E}-04$ \\
\hline 7 & $\mathrm{~N}$ & $y$ & N & $1.68 E-03$ \\
\hline
\end{tabular}

* Initial plume size for Case 1 ( width $=3.0$, height $=0.0)$. 
Table 3: 99.5 Percentile $X / Q$ s Values at Site Boundary Obtained from Acute Releases.

\begin{tabular}{|c|c|c|c|c|}
\hline $\mathrm{specif1 \textrm {c }} \mathrm{x} / \mathrm{a}$ & Buit ding Wake & Plime Meander. & Plums RIse & $x / 0\left(\mathrm{~s} / \mathrm{m}^{3}\right)$ \\
\hline 1 & $Y$ & $y$ & $y$ & $3.74 E-06$ \\
\hline 2 & $Y$ & $y$ & N & $1.40 E-05$ \\
\hline 3 & $Y$ & $\mathrm{~N}$ & N & $1.73 E-05$ \\
\hline 4 & H & $\mathrm{N}$ & N & $1.76 \mathrm{E}-05$ \\
\hline 5 & H & $Y$ & $y$ & $3.74 E-06$ \\
\hline 6 & N & $N$ & $y$ & $4.36 E-06$ \\
\hline 7 & N & $Y$ & $\mu$ & $1.41 \mathrm{E}-05$ \\
\hline
\end{tabular}

Table 4: Receptor at Highway 240, 99.5 Percentile X/Qs Values Obtained from Acute Releases.

\begin{tabular}{|c|c|c|c|c|}
\hline Speciffie $\mathrm{K} / \mathrm{O}$ & Bufllding Wake & Plume Meander & Plume Rise & $\mathrm{x} / \mathrm{Q}\left(\mathrm{s} / \mathrm{m}^{3}\right)$ \\
\hline 1 & $y$ & $y$ & $Y$ & 5.37E-06 \\
\hline 2 & $\gamma$ & $Y$ & N & 5.46E-05 \\
\hline 3 & $\gamma$ & N & N & $6.64 \mathrm{E}-05$ \\
\hline 4 & $\mathbf{N}$ & N & $N$ & $6.98 E-05$ \\
\hline 5 & N & Y & $y$ & $5.37 E-06$ \\
\hline 6 & N & N & Y & $7.03 E-06$ \\
\hline 7 & N & Y & N & $5.60 \varepsilon-05$ \\
\hline
\end{tabular}




\section{SOURCE TERM}

The source terms for the mixtures of isotopes were provided by the customer. The source terms for MFP, 12\% nominal TRU, and $23.9 \%$ PU-240 TRU for the mixtures of isotopes are shown in Tables 5, 6 and 7, respectively. For dose calculations, the source terms in the tables have been converted into specific activity for each isotope in unit of either $\mathrm{Ci}$ per $\mathrm{Ci}$ of a mixture or Ci per gram TRU.

Table 5. MFP Mixture and Percentage by Activity.

\begin{tabular}{||c|c|c||}
\hline Isotope & $\begin{array}{c}\text { Percentage by } \\
\text { Activity }\end{array}$ & Ci per Ci of Mixture \\
\hline $\mathrm{Cs}-137$ & 24.8 & 0.248 \\
\hline $\mathrm{Ba}-137 \mathrm{~m}$ & 24.8 & 0.248 \\
\hline $\mathrm{Sr}-90$ & 19.4 & 0.194 \\
\hline $\mathrm{Y}-90$ & 19.4 & 0.194 \\
\hline $\mathrm{Pu}-239$ & 0.41 & 0.0041 \\
\hline $\mathrm{Pu}-241$ & 10.4 & 0.104 \\
\hline Am-241 & 0.79 & 0.0079 \\
\hline Total & 100 & $1.00 \mathrm{Ci} / \mathrm{Ci}$ \\
\hline
\end{tabular}

Table 6. 12\% Nominal TRU and Specific Activity for Each Isotope.

\begin{tabular}{|c|c|c|c|}
\hline Isotope & $\begin{array}{c}\text { Percentage by } \\
\text { Weight }\end{array}$ & $\begin{array}{c}\text { Specific Activity } \\
\text { (Ci } / g)\end{array}$ & $\begin{array}{c}\text { Activity per } \\
\text { gram TRU (Ci) } \\
\text { (Ci) }\end{array}$ \\
\hline $\mathrm{Pu}-238$ & 0.08 & 17.4 & $1.39 \mathrm{E}-02$ \\
\hline $\mathrm{Pu}-239$ & 83.95 & 0.0613 & $5.15 \mathrm{E}-02$ \\
\hline $\mathrm{Pu}-240$ & 12.97 & 0.227 & $2.94 \mathrm{E}-02$ \\
\hline $\mathrm{Pu}-241$ & 1.10 & 103 & $1.13 \mathrm{E}+00$ \\
\hline $\mathrm{Pu}-242$ & 0.03 & 0.00394 & $1.18 \mathrm{E}-06$ \\
\hline Am-241 & 1.75 & 3.43 & $6.00 \mathrm{E}-02$ \\
\hline Tota1 & 99.9 & & $1.29+00(\mathrm{Ci} / \mathrm{g})$ \\
\hline
\end{tabular}


WHC-SD-WM-CN-016, Rev. 0

Table 7. 23.9\% Pu-240 TRU and Specific Activity for Each Isotope.

\begin{tabular}{|c|c|c|c||}
\hline Isotope & $\begin{array}{c}\text { Percentage by } \\
\text { Weight }\end{array}$ & $\begin{array}{c}\text { Specific Activity } \\
(\mathrm{C} i / g)\end{array}$ & $\begin{array}{c}\text { Activity per } \\
\text { gram TRU (Ci) } \\
(\mathrm{C} \mathrm{i})\end{array}$ \\
\hline $\mathrm{Pu}-238$ & 0.09 & 17.4 & $1.57 \mathrm{E}-02$ \\
\hline $\mathrm{Pu}-239$ & 70.60 & 0.0613 & $4.33 \mathrm{E}-02$ \\
\hline $\mathrm{Pu}-240$ & 23.86 & 0.227 & $5.42 \mathrm{E}-02$ \\
\hline $\mathrm{Pu}-241$ & 1.60 & 103 & $1.65 \mathrm{E}+00$ \\
\hline $\mathrm{Pu}-242$ & 1.30 & 0.00394 & $5.12 \mathrm{E}-06$ \\
\hline Am-241 & 2.55 & 3.43 & $8.75 \mathrm{E}-02$ \\
\hline Total & 100. & & $1.85+00(\mathrm{Ci} / \mathrm{g})$ \\
\hline
\end{tabular}

\section{UNIT DOSE CALCULATIONS}

The GENII code was used to calculate doses for each mixture of isotopes. The unit $X / Q$ and the source terms as shown in Tables 5,6 , and 7 were used as input data into the GENII code for dose calculations. Two cases for each mixture are considered: One is soluble Pu (nitrates, etc.) and the other insoluble (oxides) mixture. The calculated unit doses are shown in Table 8 for three mixtures (MFP, $12 \%$ nominal TRU and $23.9 \% \mathrm{Pu}-240$ ). The doses in the tables are expressed either in unit of $\mathrm{rem} / \mathrm{Ci}$ or $\mathrm{rem} / \mathrm{g}$.

Table 8. Doses Per Unit $X / Q$ for Soluble and Insoluble Mixtures.

\begin{tabular}{|c|c|c|}
\hline Mixture & Soluble Pu (Nitrates) & Insoluble (0xides) \\
\hline MFP & $2.0 \mathrm{E}+03(\mathrm{rem} / \mathrm{Ci})$ & $1.8 \mathrm{E}+03(\mathrm{rem} / \mathrm{Ci})$ \\
\hline TRU $(12 \%$ Nominal) & $2.5 \mathrm{E}+04(\mathrm{rem} / \mathrm{g})$ & $2.0 \mathrm{E}+04(\mathrm{rem} / \mathrm{g})$ \\
\hline TRU $(23.9 \% \mathrm{Pu}-240)$ & $3.3 \mathrm{E}+04(\mathrm{rem} / \mathrm{g})$ & $2.7 \mathrm{E}+04(\mathrm{rem} / \mathrm{g})$ \\
\hline
\end{tabular}




\section{REFERENCES}

Hey, B. E., 1994, GXQ Program Users' Guide, WHC-SD-GN-SWD-30002, Westinghouse Hanford Company, Richland, Washington.

Napier, B. A., et a1., 1988, GENII - The Hanford Environmental Radiation Dosimetry Software System, PNL-6584, Pacific Northwest Laboratory, Richland, Washington.

NRC, 1988, Nuclear Fuel Cycle Facility Accident Analys is Handbook. NUREG 1320. Nuclear Regulatory Commission, Washington, D.C.

NRC, 1990, MELCOR Accident Consequence Code System (MACCS), Model Description, NUREG.CR-4691, SAND86-1562, Vol. 2, U.S. Nuclear Regulatory Commission, Washington, D.C.

Randerson, D., ed., 1984, Atmospheric Science and Power Production, DOE/TIC27601 (DE84005177), Technical Information Center, Office of Scientific and Technical Information Center, U.S. Department of Energy, Washington, D.C.

U.S. Nuclear Regulatory Commission, November 1982, Atmospheric Dispersion Models For Potential Accident Consequence Assessments At Nuclear Power

Plants, Regulatory Guide 1.145, Revision 1, Office of Nuclear Regulatory Research. 
WHC-SD-WM-CN-016, Rev. 0

APPENDIX A

RECEPTOR LOCATION AND SOURCE TERM PROVIDED BY THE CUSTOMER 
WHC-WM-CN-016, Rev.0

WHC-SD-WM-TI-738, Rev.0

Solid Waste Safety Analysis

$376-1941$ A2-26

January 10. 1996

GENII CALCULATIONS EOR THE CGNTRAL WASTE COMPLEX INTERIM SAFETY BASIS EFFORT

J.C. Van Reuren

$c s:$
A3 -34

T3-0I

T3-0I

It is raquested that $X / Q$ and unit dose consequence detanminarions be made for the Central waste Comolex, which now includes the buildings proposed for the N-I12 project. It is preferred that the MaCCs code be used for che $X / Q$ determination, eliminating the need for the analyst to evaluate time distributions associated with the scenario.

The onsite dose receptor is considered to reside at the Plutonium Einishing plant. The exact distance is expected to be extracted from site drawings. The location of the Long Term Drum Storage buildings for project $N-112$ are assumed as being in a general location not affecting the onsite receptor distance. The plume difection is assumed to be in a east-souch-east direceion.

Other recepcor locations need to be considered (i.e., iighway-240 and $11.7 \mathrm{~km}$ in a west direction).

The effort will need $\mathrm{X} / Q$ for scenarios with and without plume meander. Eurchermore, there is a need for $X / Q$ s with and without building wake considerations. The building wake considerations will be developed from dimensions that reflect liose for the 2402 buildings and will be supolied to you. Finally analyses demonstrate the need for plume fise decermination for the scenarios using burning diesel.

Therefore, the efiort wiII require seven potencial $\mathrm{G} / \mathrm{QS}$ for the different seceptors as shown in the following matrix:

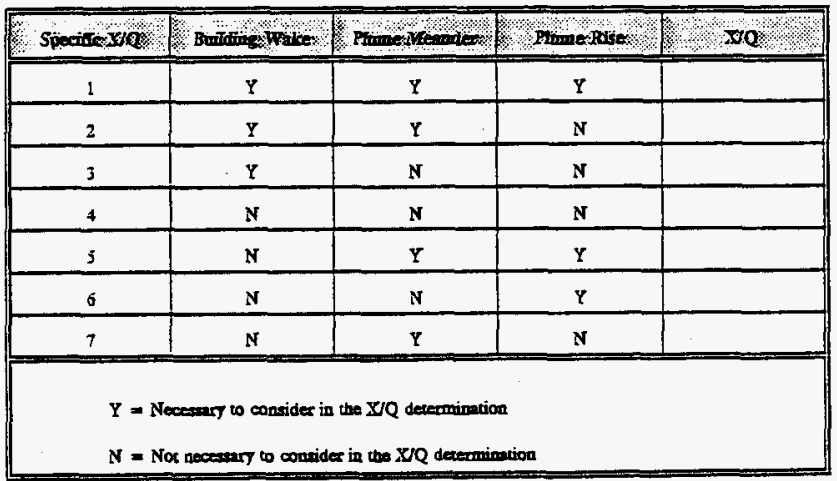


Unit doses consequence based on Plutonium-Equivalent Curie (PE-Ci) quantities are necessary for different isocopic mixtures used in the safery analyses.

Calculations are to be based upon inhalation. The following isocopic distributions are noted for the storage facilities.

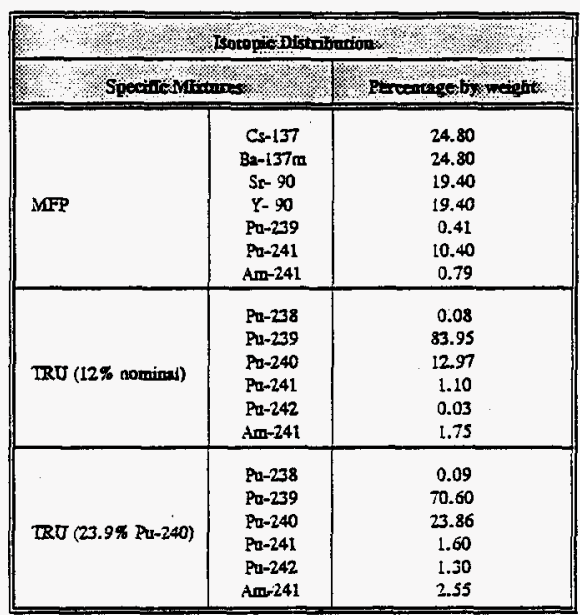


WHC-SD-WM-CN-016, Rev. 0

APPENDIX B

X/Q OUTPUT FILES

12 . 
Gxa Version 4.0

Decenber 19, 1994

General Purpose Atmospheric Dispersion Code

Produced by West inghouse Hanford Company

Users Guide documented in WHC-SD-GN-SWD-30002 Rev. 1.

Validation documented in WHC-SD-GN-SWD-30003 Rev. 1.

Code Custodian is: Brit E. Hey

West inghouse Hanford Company

P.O. Box 1970

Richland WA 99352

(509) $376-2921$

Run Date $=03 / 13 / 96$

Run Time $=13: 40: 58.77$

INPUT ECHO:

CWC Sol id Waste Storage P4 to P7 XQs, case 1.

c GXQ Version 4.0 input file

c mode

1

COOE CHOICE:

$c$ mode $=1$ then $X / Q$ based on Hanford site specific meteorology

$c$ mode $=2$ then $X / Q$ based on atmospheric stability $c$ lass and wind speed

$c$ mode $=3$ then $x / 0$ plat file is created

C LOGICAL CHOICES:

c fox inorm icdf ichk isite ipop

$c$ ifox $=t$ then joint frequency used to compute frequency to exceed $X / Q$

$c \quad=f$ then joint frequency used to compute annual average $X / Q$

$c$ inorm $=t$ then joint frequency data is normalized (as in GENII)

$c \quad=f$ then joint frequency data is un-normalized

$c$ icdf $=t$ then cumulative distribution file created (CDF.OUT)

$c=f$ then no cumulative distribution file created

$c$ ichk $=t$ then $X / Q$ parameter print option turned on

$=f$ then no parameter print

c isite $=t$ then $x / 0$ based on joint frequency data for all 16 sectors

$=f$ then $X / Q$ based on joint frequency data of individual sectors

ipop $=t$ then $X / Q$ is population weighted

$=f$ then no population weighting

$c X / Q$ AND WIND SPEED ADJUSTMENT MODELS:

c ipuff idep isrc iwind

0 ipuff 0 idep 0

c DIFFUSION COEFFICIENT ADJUSTMENT MODELS:

c iwake ipm iflow ientr

c EFFECTIVE RELEASE HEIGHT ADJUSTMENT MODELS:

c (irise igrnd) iwash igrav

$c$ ipuff $=1$ then $x / 0$ calculated using puff model

$c \quad=0$ then $X / Q$ calculated using defaul $t$ cont inuous plume model

$c$ idep $=1$ then plume depletion model turned on (Chamberlain model)

$c$ isrc $=1$ then $x / Q$ multiplied by scalar

$c \quad=2$ then $X / Q$ adjusted by wind speed function

$c$ iwind = 1 then wind speed corrected for plume height

$c$ iwake $=1$ then NRC RG 1.145 building wake model turned on

c $\quad 2$ then maccs virtual distance building wake model turned on

$c$ ipm $=1$ then MRC RG 1.145 plume meander model turned on

c $\quad 2$ then 5 th Power Law plume meander model turned on

c $\quad=3$ then sector average model turned on

c iflow $=1$ then sigmas adjusted for volume flow rate

c ientr = 1 then method of Pasquill used to account for entrainment

c irise $=1$ then MACCS buoyant plume rise model turned on

$c \quad=2$ then ISC2 monentun/buoyancy piume rise model turned on

c igrnd $=1$ then Mills buoyant plume rise modification for ground effects 
i wash = 1 then stack downwash model turned on

$c$ igrav $=1$ then gravitational settling model turned on

$=0$ unless specified otherwise, 0 turns model off

c PARAMETER INPUT:

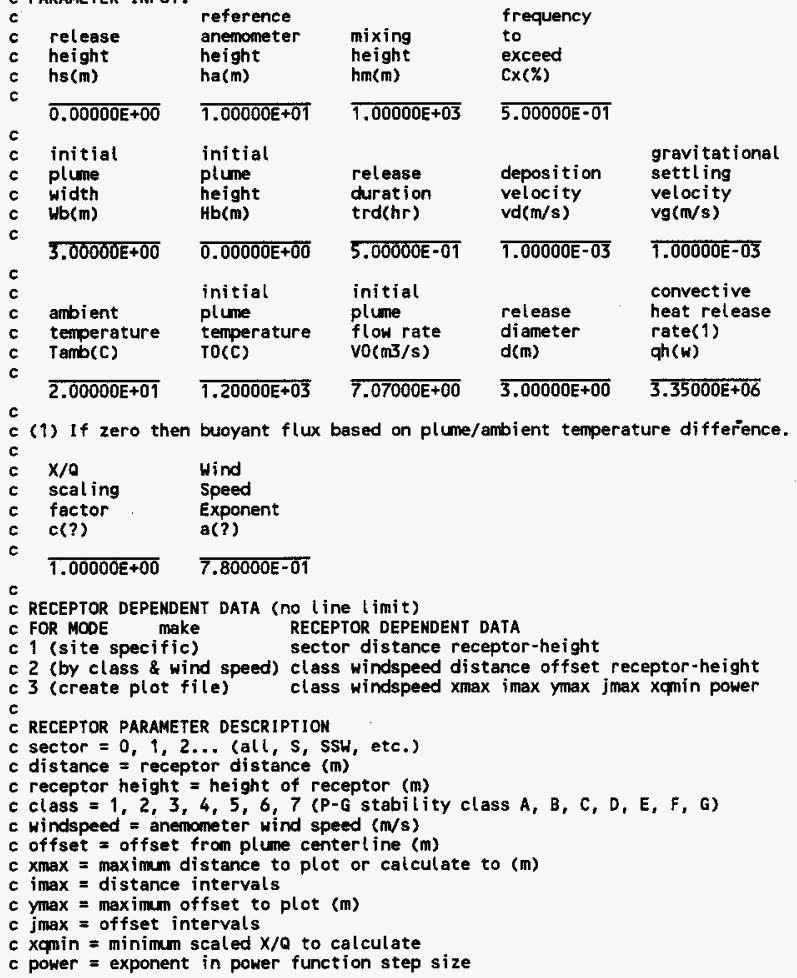

\section{rel ease} chiration trd(hr)

deposition velocity 5.00000E-0

1.00000 E-03

initial

plume

flow rate Vo $(\mathrm{m} 3 / \mathrm{s})$

release

diameter

d(m)

gravi tational settling velocity $\mathrm{vg}(\mathrm{m} / \mathrm{s})$

$2.00000 \mathrm{E}+01$

$\overline{1.20000 E+03}$

$\overline{7.07000 E+00}$

$\overline{3.00000 E+00}$

$1.00000 \mathrm{E}-03$

convective

$c$ (1) If zero then buoyant flux based on plume/ambient temperature difference.

$x / 0 \quad$ Wind

scaling Speed

factor. Exponent

c(?) $\quad a(?)$

$\overline{1.00000 E+00} \overline{7.80000 E-01}$

C RECEPTOR DEPENDENT DATA (no line limit)

C FOR MODE make RECEPTOR DEPENDENT DATA

c 1 (site specific) sector distance receptor-height

c 2 (by class \& wind speed) class windspeed distance of fset receptor-height

c 3 (create plot file) class windspeed $x \max i \max$ ymax jmax xqmin power

C RECEPTOR PARAMETER DESCRIPTION

c sector $=0,1,2 \ldots$ (all, s, SSH, etc.)

c distance $=$ receptor distance $(\mathrm{m})$

c receptor height $=$ height of receptor (m)

$c$ class $=1,2,3,4,5,6,7$ (P-G stability class $A, B, C, D, E, F, G$ )

c windspeed $=$ anemometer wind speed $(\mathrm{m} / \mathrm{s})$

$c$ offset $=$ offset from plume centertine (m)

c $x \max =$ maximm distance to plot or calculate to $(\mathrm{m})$

$c$ imax $=$ distance intervals

c ymax $=$ maximum offset to plot (m)

$c$ jmax $=$ offset intervals

c $x q m i n=$ minimum scaled $x / Q$ to calculate

$c$ power $=$ exponent in power function step size

MOOE:

Site specific $X / Q$ calculated.

LOGICAL CHOICES:

Joint frequency used to calculate $X / Q$ based on frequency of exceedance.

No normalization of joint frequency.

$x / Q$ calculated for single sector.

MOOELS SELECTED:

MACCS Virtual source building wake model selected.

5 th power law pl une meander model selected.

MACCS buoyancy plume rise model based on convective heat.

Mills buoyant rise modification for pool fire selected.

Defaut t Gaussian plume model selected.

Wind velocity corrected for average plume height.

WARNING/ERROR MESSAGES:

WARNING \#10 - X/Q may be underestimated for plume rise scenario without accounting for entrainment.

JOINT FREQUENCY DATA: 
WHC-SD-WM-CN-016, Rev. 0

200 AREA (HMS) - $10 \mathrm{M}$ - Pasquill A - G (1983 - 1991 Average)

Created $8 / 26 / 92$ KR

CWC Solid Waste Storage P4 to P7 XOS, case 1.

\begin{tabular}{|c|c|c|c|c|c|c|c|c|}
\hline SECTOR & $\begin{array}{l}\text { DISTANCE } \\
(\mathrm{m})\end{array}$ & $\begin{array}{l}\text { RECEPT } \\
\text { HEIGKT } \\
\text { (m) }\end{array}$ & $\begin{array}{l}\text { SECT. } \\
\text { FREQ. } \\
(\%)\end{array}$ & POPULATION & $\begin{array}{l}\text { TOTAL } \\
\text { POPULATION } \\
\text { SCALED } \\
\times / a \\
\left(\mathrm{~s} / \mathrm{m}^{3}\right)\end{array}$ & $\begin{array}{l}\text { AVERAGE } \\
\text { IND IVIDUAL } \\
\text { SCALED } \\
x / Q \\
(\mathrm{~s} / \mathrm{m} 3)\end{array}$ & $\begin{array}{l}\text { ATM. } \\
\text { STAA. } \\
\text { CLASS }\end{array}$ & $\begin{array}{l}\text { WIND } \\
\text { SPEED } \\
\text { (m/s) }\end{array}$ \\
\hline $\begin{array}{l}\text { ESE } \\
W \\
W\end{array}$ & $\begin{array}{r}498 \\
11700 \\
4070\end{array}$ & 0 & $\begin{array}{r}18.80 \\
4.80 \\
4.80\end{array}$ & $\begin{array}{l}1 \\
1 \\
1\end{array}$ & $\begin{array}{l}3.13 E-05 \\
3.74 E-06 \\
5.37 E-06\end{array}$ & $\begin{array}{l}3.13 E-05 \\
3.74 E-06 \\
5.37 E-06\end{array}$ & $\begin{array}{l}D \\
F \\
F\end{array}$ & $\begin{array}{l}9.80 \\
0.89 \\
2.65\end{array}$ \\
\hline
\end{tabular}


GXa Version 4.0

December 19, 1994

General Purpose Atmospheric Dispersion Code

Produced by Westinghouse Hanford Company

Users Guide documented in WHC-SD-GN-SWD-30002 Rev. 1.

Validation documented in WHC-SD-GN-SWD-30003 Rev. 1.

Code Custodian is: Brit E. Hey

Westinghouse Hanford Company

P.0. Box 1970

Richland, WA 99352

(509) 376-2921

Run Date $=03 / 10 / 96$

Run Time $=11: 38: 27.76$

INPUT ECHO:

CWC Solid Waste storage P4 to P7 XQs, case 2 with wake effect \& plume meander c GXa Version 4.0 Input File

c mode

1

C MODE CHOLCE:

$c$ mode $=1$ then $X / Q$ based on Hanford site specific meteorology

$c$ mode $=2$ then $X / Q$ based on atmospheric stability class and wind speed

$c$ mode $=3$ then $X / Q$ plot file is created

C LOGICAL CHOICES:

c ifox inorm icdf ichk isite ipop

$T$ F F.' $F$ F F

c ifox $=$ t then joint frequency used to compute frequency to exceed $x / 0$

$c \quad=f$ then joint frequency used to compute annual average $X / a$

c inorm $=t$ then joint frequency data is normalized (as in GENI!)

$c \quad=f$ then joint frequency data is un-normalized

$c$ icdf $=t$ then cumulative distribution file created (CDF.OUT)

$=f$ then no cumulative distribution file created

c $i_{\text {chk }}=\mathrm{t}$ then $\mathrm{X} / Q$ parameter print option turned on

$c$ a then no parameter print

$c$ isite $=t$ then $x / Q$ based on joint frequency data for alt 16 sectors

$c \quad=f$ then $X / Q$ based on joint frequency data of individual sectors

$c$ ipop $=t$ then $X / Q$ is population weighted

$c \quad=f$ then no population weighting

C X/Q AND WIND SPEED ADJUSTMENT MODELS:

C ipuff idep isre iwind

c DIFFUSION COEFFICIENT ADJUSTMENT MODELS:

C iwake ipm iflow ientr.

c EFFECTIVE RELEASE HEIGHT ADJUSTMENT MOOELS :

c (irise igrnd) i wash igrav

$\begin{array}{llll}0 & 0 & 0 & 0\end{array}$

c ipuff $=1$ then $X / Q$ calculated using puff model

c $=0$ then $X / Q$ calculated using default continuous plume model

c idep $=1$ then plune depletion model turned on (Chanberlain model)

$c$ isrc $=1$ then $X / Q$ multiplied by scalar

$c \quad=2$ then $X / Q$ adjusted by wind speed function

iwind $=1$ then wind speed corrected for plume height

iwake $=1$ then MRC RG 1.145 building wake model turned on

$c \quad=2$ then MACCS virtual distance building wake model turned on

ipm = 1 then NRC RG 1.145 plume meander model turned on

$c=2$ then 5 th Power Law plume meander model turned on

c $\quad=3$ then sector average model turned on 


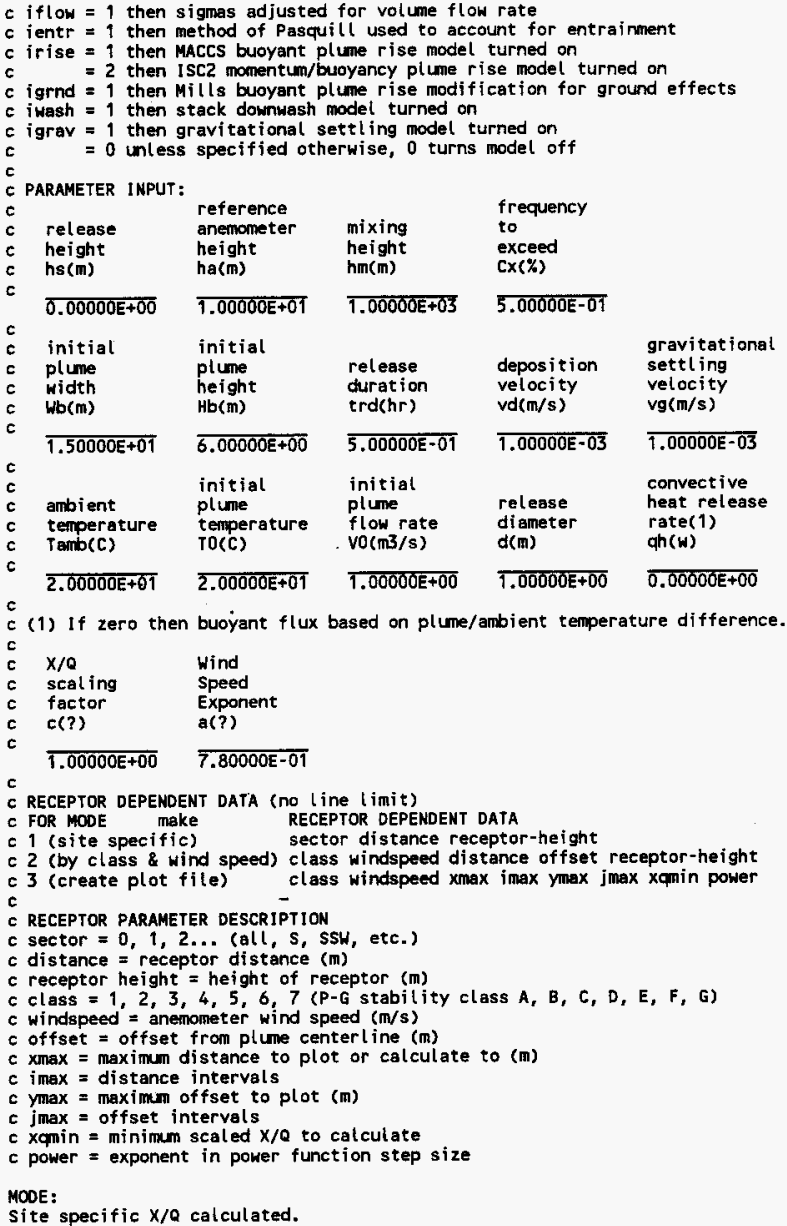

Site specific $X / Q$ calculated.

LOGICAL CHOICES:

Joint frequency used to calculate $x / a$ based on frequency of exceedance. No normalization of joint frequency.

$X / Q$ calculated for single sector.

MODELS SELECTED:

MACCS Virtual source building wake modei selected.

5 th power law plume meander model selected.

Default Gaussian plume model selected.

WARNING/ERROR MESSAGES:

JOINT FREQUENCY DATA: 
WHC-SD-WM-CN-016, Rev. 0

200 AREA (HMS) - $10 \mathrm{M}$ - Pasquill A - G (1983 - 1991 Average)

Created $8 / 26 / 92$ KR

CWC Solid Waste Storage P4 to P7 XQs, case 2 with wake effect \& plume meander

\begin{tabular}{|c|c|c|c|c|c|c|c|c|}
\hline ECTOR & $\begin{array}{l}\text { DISTANCE } \\
\text { (m) }\end{array}$ & $\begin{array}{l}\text { RECEPT } \\
\text { HEIGHT } \\
\text { (m) }\end{array}$ & $\begin{array}{l}\text { SECT. } \\
\text { FREQ. } \\
(\%)\end{array}$ & POPULATION & $\begin{array}{l}\text { TOTAL } \\
\text { POPULATION } \\
\text { SCALED } \\
X / Q \\
(s / m 3)\end{array}$ & $\begin{array}{l}\text { AVERAGE } \\
\text { INDIVIDUAL. } \\
\text { SCALED } \\
X / Q \\
(\mathrm{~s} / \mathrm{m3})\end{array}$ & $\begin{array}{l}\text { ATM. } \\
\text { STAB. } \\
\text { CLASS }\end{array}$ & $\begin{array}{l}\text { WIND } \\
\text { SPEED } \\
(\mathrm{m} / \mathrm{s})\end{array}$ \\
\hline $\begin{array}{l}\overline{\text { ESE }} \\
\mathrm{H}\end{array}$ & $\begin{array}{r}498 \\
11700 \\
4070\end{array}$ & $\begin{array}{l}0 \\
0 \\
0\end{array}$ & $\begin{array}{r}18.80 \\
4.80 \\
4.80\end{array}$ & $\begin{array}{l}7 \\
1 \\
1\end{array}$ & $\begin{array}{l}1.36 \mathrm{E}-03 \\
1.40 \mathrm{E}-05 \\
5.46 \mathrm{E}-05\end{array}$ & $\begin{array}{l}1.36 \mathrm{E}-03 \\
1.40 \mathrm{E}-05 \\
5.46 \mathrm{E}-05\end{array}$ & $\begin{array}{l}\bar{F} \\
G \\
G\end{array}$ & $\begin{array}{l}0.89 \\
2.65 \\
2.65\end{array}$ \\
\hline
\end{tabular}


GXQ Version 4.0

December 19, 1994

General Purpose Atmospheric Dispersion Code Produced by Westinghouse Hanford Company

Users Guide documented in WHC-SO-GN-SWO-30002 Rev. 1.

Val idation documented in WHC-SD-GN-SWD-30003 ReV. 1.

Code Custodian is: Brit E. Hey

West inghouse Hanford Company

P.0. Box 1970

Richland, WA 99352

(509) $376-2921$

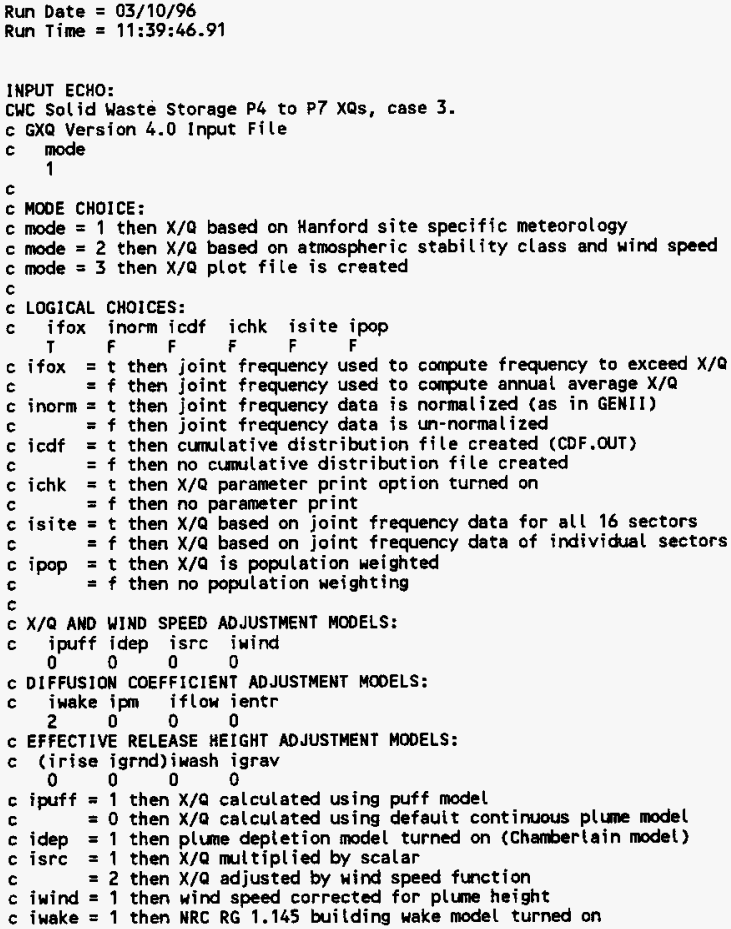


$c \quad=2$ then MAccs virtual distance building wake model turned on

$c$ ipm = 1 then NRC RG 1.145 plume meander model turned on

$c \quad=2$ then 5 th Power Law plume meander model turned on

c $\quad=3$ then sector average model turned on

$c$ iflow = 1 then sigmas adjusted for volume flow rate

$c$ ientr $=1$ then method of Pasquill used to account for entrainment

c irise $=1$ then Maccs buoyant plume rise model turned on

c $=2$ then ISC2 momentum/buoyancy plume rise model turned on

c igrnd $=1$ then Mills buoyant plume rise modification for ground effects

c iwash $=1$ then stack downwash model turned on

c igrav = 1 then gravitational settling model turned on

c $\quad 0$ unless specified otherwise, 0 turns model off

PARAMETER INPUT:

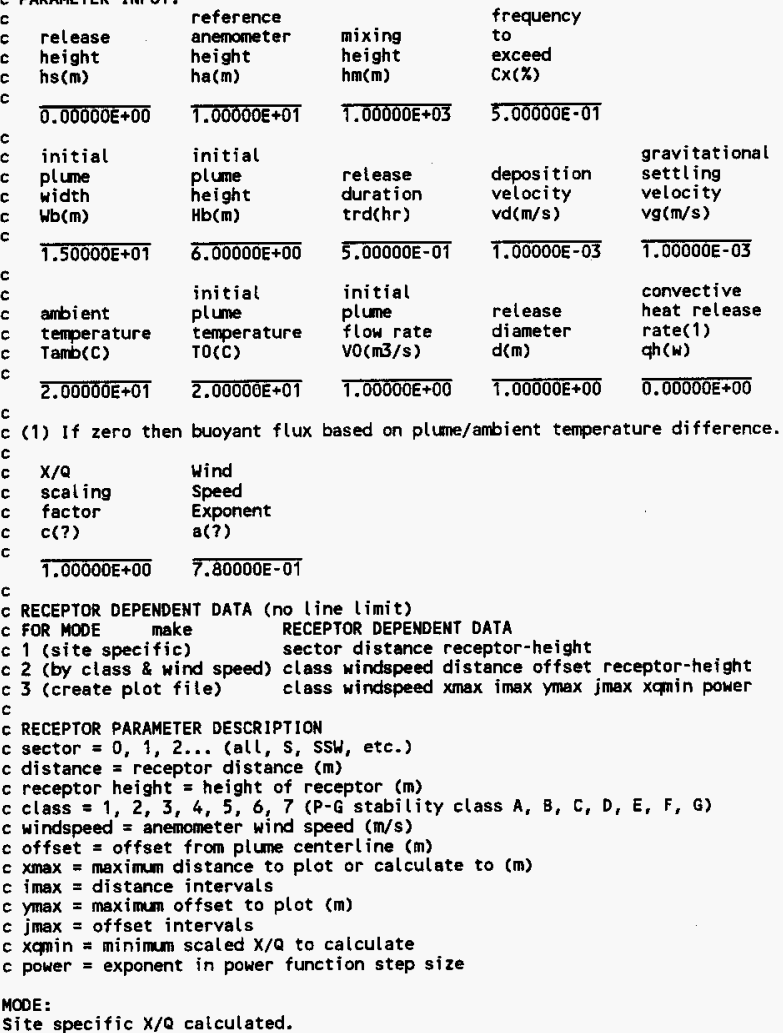

Site specific $\times 10$ calculated.

LOGICAL CHOICES:

Joint frequency used to calculate $x / Q$ based on frequency of exceedance.

Ho normalization of joint frequency.

$X / Q$ calculated for single sector.

MOOELS SELECTED:

Maccs virtual source building wake model selected.

Default Gaussian plume model selected. 
WHC-SD-WM-CN-016, Rev. O

WARNING/ERROR MESSAGES:

JOINT FREQUENCY DATA:

200 AREA (HMS) - $10 \mathrm{M}$ - Pasquill A - G (1983 - 1991 Average)

Created $8 / 26 / 92 \mathrm{KR}$

CWC solid Waste storage P4 to P7 XQs, case 3 .

\begin{tabular}{|c|c|c|c|c|c|c|c|c|}
\hline SECTOR & $\begin{array}{l}\text { DISTANCE } \\
\text { (m) }\end{array}$ & $\begin{array}{l}\text { RECEPT } \\
\text { HEIGHI } \\
\text { (m) }\end{array}$ & $\begin{array}{l}\text { SECT. } \\
\text { FREQ. } \\
\text { (X) }\end{array}$ & POPULATION & $\begin{array}{c}\text { TOTAL } \\
\text { POPULATION } \\
\text { SCALED } \\
\text { X/Q } \\
(\mathrm{s} / \mathrm{mB})\end{array}$ & $\begin{array}{l}\text { AVERAGE } \\
\text { INDIVIDUAL } \\
\text { SCALED } \\
\text { X/Q } \\
(\mathrm{s} / \mathrm{m} 3)\end{array}$ & $\begin{array}{l}\text { ATM. } \\
\text { STAB. } \\
\text { CLASS }\end{array}$ & $\begin{array}{l}\text { WIND } \\
\text { SPEED } \\
\text { (m/s) }\end{array}$ \\
\hline ESE & $\begin{array}{r}498 \\
11700 \\
4070\end{array}$ & 0 & $\begin{array}{r}18.80 \\
4.80 \\
4.80\end{array}$ & $\begin{array}{l}1 \\
1\end{array}$ & $\begin{array}{l}1.47 \mathrm{E}-03 \\
1.73 \mathrm{E}-05 \\
6.64 \mathrm{E}-05\end{array}$ & $\begin{array}{l}1.47 \mathrm{E}-03 \\
1.73 \mathrm{E}-05 \\
6.64 \mathrm{E}-05\end{array}$ & $\begin{array}{l}F \\
G \\
G\end{array}$ & $\begin{array}{l}0.89 \\
2.65 \\
2.65\end{array}$ \\
\hline
\end{tabular}


QXa Version 4.0

Decenber 19, 1994

General Purpose Atmospheric Dispersion Code Produced by Westinghouse Hanford Company

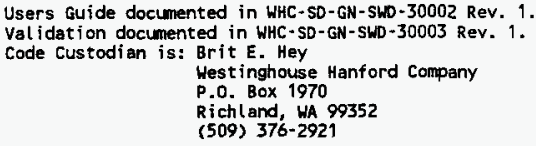

Run Date $=03 / 10 / 96$

Run Time $=09: 58: 47.97$

INPUT ECHO:

CWC Solid Waste Storage $\mathrm{P4}$ to $\mathrm{P7} \times 0 \mathrm{Os}$, case 4 . no effect c. GXa Version 4.0 Input file

c. mode

$c$

C MODE CHOICE:

c mode $=1$ then $X / Q$ based on Hanford site specific meteorology

$c$ mode $=2$ then $X / Q$ based on atmospheric stability $c$ lass and wind speed $c$ mode $=3$ then $X / Q$ plot file is created

c

C LOGICAL CHOICES:

c ifox inorm icdf ichk isite ipop

$c$ ifox $=t$ then joint frequency used to compute frequency to exceed $x / Q$

$c \quad=f$ then joint frequency used to compute annual average $x / 0$

$c$ inorm $=t$ then joint frequency data is normal ized (as in GENI1)

$c \quad=f$ then joint frequency data is un-normal ized

$c$ icdf $=t$ then cumulative distribution file created (CDF.OUT)

c $=f$ then no cumulative distribution file created

c ichk $=t$ then $x / O$ parameter print option turned on

$c \quad=f$ then no parameter print

$c$ isite $=t$ then $X / Q$ based on joint frequency data for all 16 sectors

$c \quad=f$ then $X / Q$ based on joint frequency data of individual sectors

$c$ ipop $=t$ then $X / Q$ is population weighted

c $\quad=f$ then no population weighting

c X/Q AND WIHD SPEED ADJUSTMEHT MODELS:

c ipuff idep isre iwind

$\begin{array}{llll}1 & 0 & 0 & 0\end{array}$

c DIFFUSION COEFFICIENT ADJUSTMENT MODELS:

c iwake ipm iflow ientr

0 O 0 O 0

c EFFECTIVE RELEASE HEIGHT ADJUSTMENT MODELS:

c (irise igrnd) iwash igrav

0 0 0 o 0

c ipuff $=1$ then $X / 0$ calculated using puff model

c $\quad=0$ then $X / Q$ calculated using defaul $t$ continuous plume model

$c$ idep $=1$ then plume depletion model turned on (Chamberlain model)

c isrc $=1$ then $X / Q$ multiplied by scalar

$c \quad=2$ then $X / Q$ adjusted by wind speed function

$c$ iwind $=1$ then wind speed corrected for plume height

c $i$ wake $=1$ then NRC RG 1.145 building wake model turned on

c $=2$ then MACCS virtual distance building wake model turned on 
WHC-SD-WM-CN-016, Rev. 0

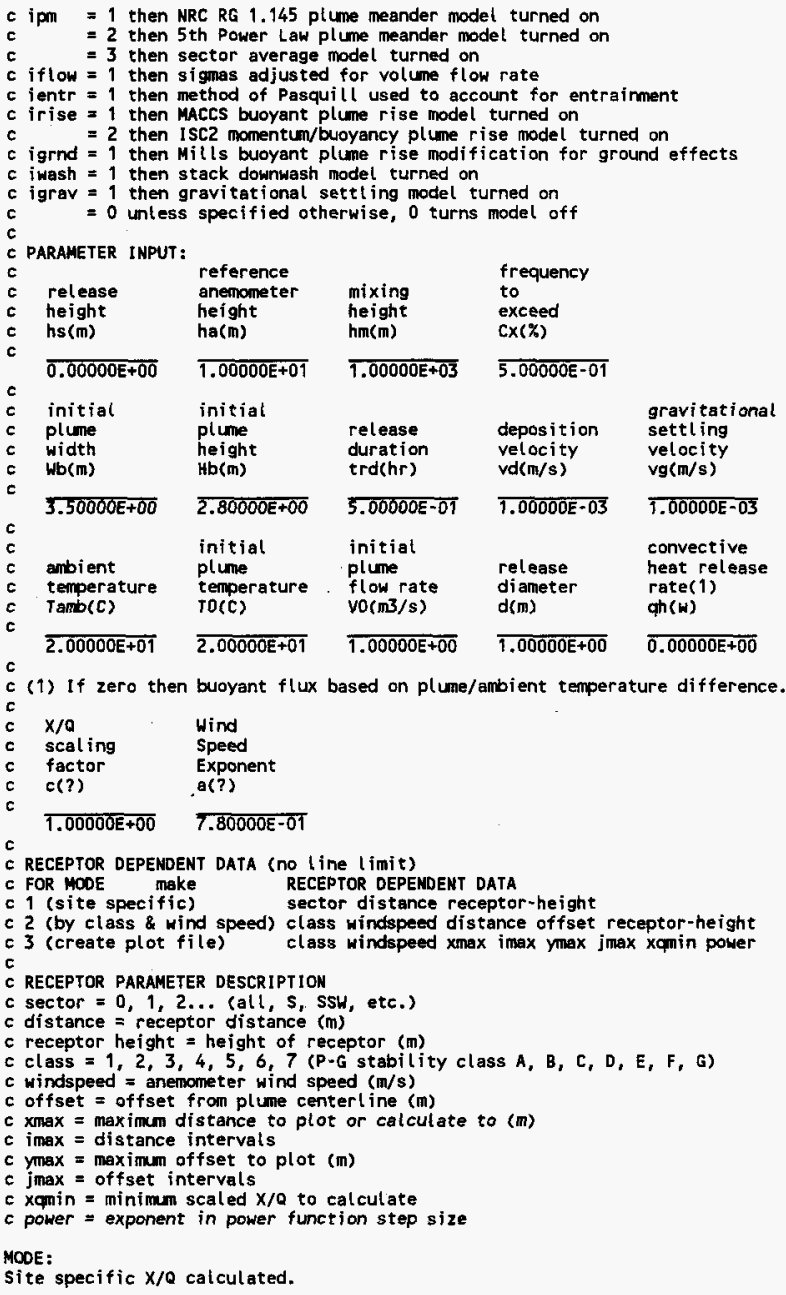

LOGICAL CHOICES:

Joint frequency used to calculate $X / Q$ based on frequency of exceedance.

No normalization of joint frequency.

$x / Q$ calculated for single sector.

MOOELS SELECTED:

Default Gaussian plume model selected.

WARNING/ERROR MESSAGES: 
WHC-SD-WM-CN-016, Rev. 0

JOINT FREQUENCY DATA:

200 AREA (HMS) - $10 \mathrm{M}$ - Pasquill A - G (1983 - 1991 Average)

Created $8 / 26 / 92$ KR

CWC Solid Waste Storage P4 to P7 XQs, case 4. no effect

\begin{tabular}{|c|c|c|c|c|c|c|c|c|}
\hline SECTOR & $\begin{array}{c}\text { DISTANCE } \\
(\mathrm{m})\end{array}$ & $\begin{array}{c}\text { RECEPT } \\
\text { HEIGHT } \\
(m)\end{array}$ & $\begin{array}{l}\text { SECT, } \\
\text { FREQ. } \\
(X)\end{array}$ & POPULATION & $\begin{array}{l}\text { TOTAL } \\
\text { POPULATION } \\
\text { SCALED } \\
\text { X/Q } \\
(\mathrm{s} / \mathrm{m} 3)\end{array}$ & $\begin{array}{l}\text { AVERAGE } \\
\text { INDIVIDUAL } \\
\text { SCALED } \\
X / Q \\
(\mathrm{~s} / \mathrm{m} 3)\end{array}$ & $\begin{array}{l}\text { ATM. } \\
\text { STAB. } \\
\text { CLASS }\end{array}$ & $\begin{array}{l}\text { W1ND } \\
\text { SPEED } \\
(\mathrm{m} / \mathrm{s})\end{array}$ \\
\hline $\begin{array}{l}\text { ESE } \\
W \\
\text { S } \\
\text { SSW } \\
\text { SW } \\
\text { WSW } \\
W \\
\text { WNW } \\
\text { NW }\end{array}$ & $\begin{array}{r}498 \\
11700 \\
5550 \\
4810 \\
4160 \\
4160 \\
4070 \\
6660 \\
8140\end{array}$ & $\begin{array}{l}0 \\
0 \\
0 \\
0 \\
0 \\
0 \\
0 \\
0 \\
0\end{array}$ & $\begin{array}{r}18.80 \\
4.80 \\
6.30 \\
4.53 \\
2.93 \\
2.72 \\
4.80 \\
3.98 \\
4.72\end{array}$ & $\begin{array}{l}1 \\
1 \\
1 \\
1 \\
1 \\
1 \\
1 \\
1 \\
1\end{array}$ & $\begin{array}{l}2.10 E-03 \\
1.76 E-05 \\
2.50 E-05 \\
2.21 E-05 \\
2.90 E-05 \\
2.91 E-05 \\
6.98 E-05 \\
2.05 E-05 \\
2.89 E-05\end{array}$ & $\begin{array}{l}2.10 \mathrm{E}-03 \\
1.76 \mathrm{E}-05 \\
2.50 \mathrm{E}-05 \\
2.21 \mathrm{E}-05 \\
2.90 \mathrm{E}-05 \\
2.91 \mathrm{E}-05 \\
6.98 \mathrm{E}-05 \\
2.05 \mathrm{E}-05 \\
2.89 \mathrm{E}-05\end{array}$ & $\begin{array}{l}F \\
G \\
E \\
F \\
F \\
F \\
G \\
G \\
G\end{array}$ & $\begin{array}{l}0.89 \\
2.65 \\
0.89 \\
2.65 \\
2.65 \\
2.65 \\
2.65 \\
4.70 \\
2.65\end{array}$ \\
\hline
\end{tabular}


WHC-SD-WM-CN-016, Rev. 0

Qxo version 4.0

Decenber 19, 1994

General Purpose Atmospheric Dispersion Code

Produced by West inghouse Henford Company

Users Guide documented in HHC-SD-GN-SWD-30002 Rev. 1.

Validation documented in WHC-SD-GN-SWD-30003 Rev. 1.

Code Custodian is: Brit $\varepsilon$. Hey

West inghouse Hanford Company

P.O. Box 1970

Richland, WA 99352

(509) $376-2921$

Run Date $=03 / 13 / 96$

Run Time $=13: 48: 01.48$

INPUT ECHO:

CWC Solid Waste Storage P4 to P7 Xas, case 5.

c GXQ Version 4.0 Input file

c mode

1

$c$

c MODE CHOICE:

$c$ mode $=1$ then $X / Q$ based on Hanford site specific meteorology

$c$ mode $=2$ then $X / Q$ based on atmospheric stability class and wind speed

$c$ mode $=3$ then $X / Q$ plot file is created

$c$

c LOGICAL CHOICES:

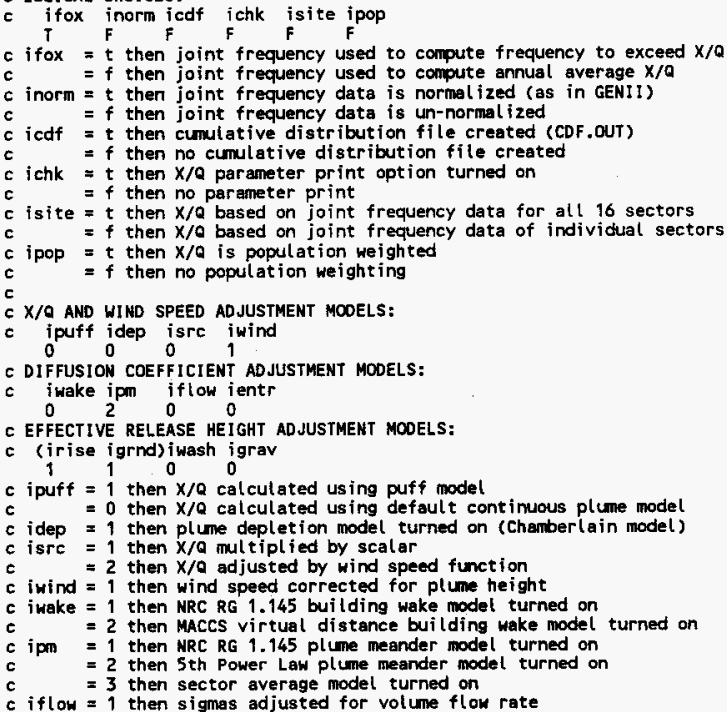




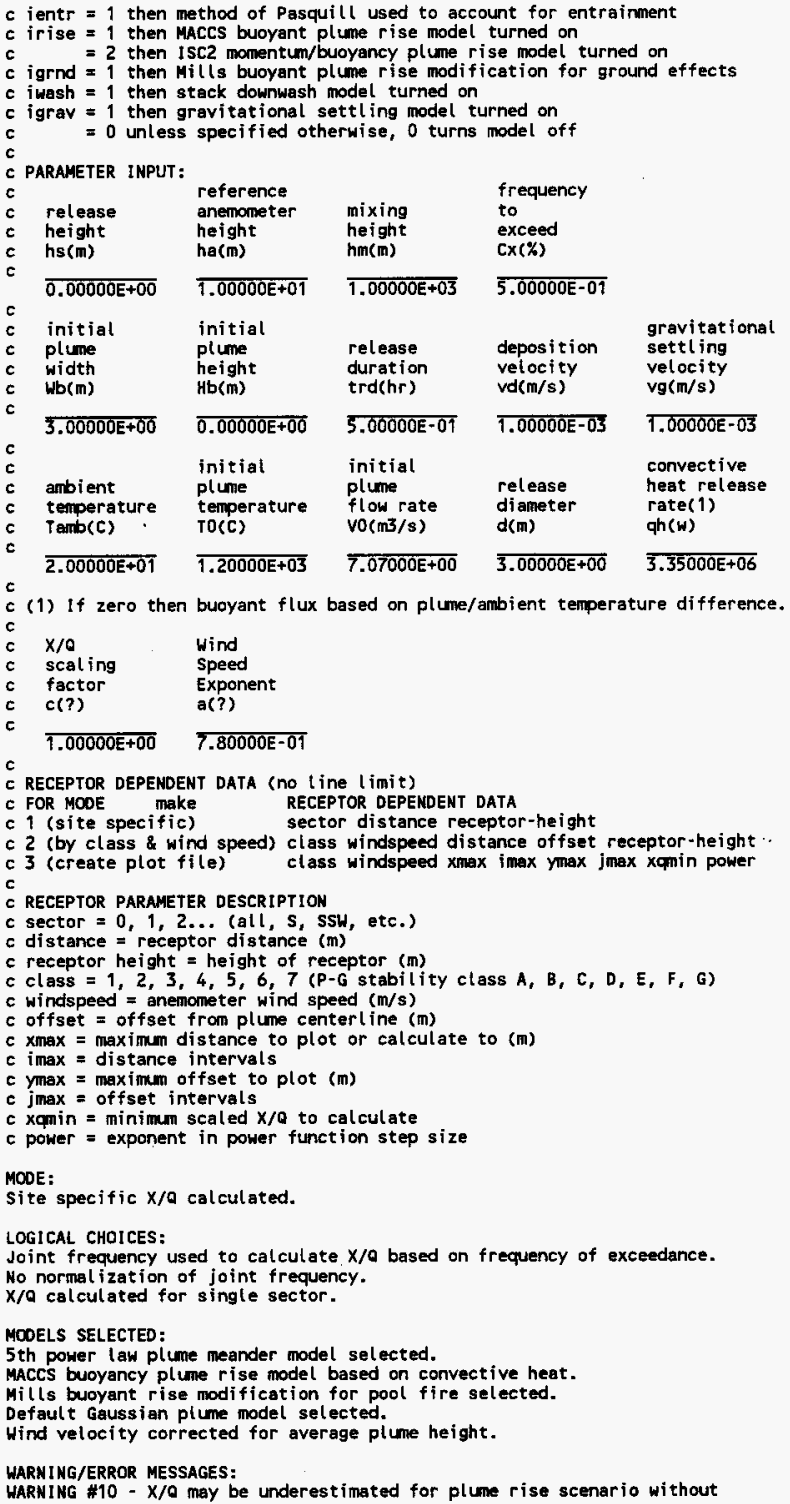


WHC-SD-WM-CN-016, Rev. 0

accounting for entrainment.

JOINT FREQUENCY DATA:

200 AREA (HMS) - 10 - Pasquill A - G (1983 - 1991 Average)

Created 8/26/92 KR

CWC Solid Waste Storage P4 to P7 XQS, case 5 .

\begin{tabular}{|c|c|c|c|c|c|c|c|c|}
\hline SECTOR & $\begin{array}{l}\text { DISTANCE } \\
\text { (m) }\end{array}$ & $\begin{array}{l}\text { RECEPT } \\
\text { HEIGHT } \\
(m)\end{array}$ & $\begin{array}{l}\text { SECT. } \\
\text { FREQ. } \\
(X)\end{array}$ & POPULATION & $\begin{array}{l}\text { TOTAL } \\
\text { POPULATION } \\
\text { SCALED } \\
X / Q \\
\left(\mathrm{~s} / \mathrm{m}^{3}\right)\end{array}$ & $\begin{array}{l}\text { AVERAGE } \\
\text { INDIVIDUAL } \\
\text { SCALED } \\
X / Q \\
(\mathrm{~s} / \mathrm{m} 3)\end{array}$ & $\begin{array}{l}\text { ATM. } \\
\text { STAB. } \\
\text { CLASS }\end{array}$ & $\begin{array}{l}\text { WIND } \\
\text { SPEED } \\
(\mathrm{m} / \mathrm{s})\end{array}$ \\
\hline $\begin{array}{l}\text { ESE } \\
W \\
W\end{array}$ & $\begin{array}{r}498 \\
11700 \\
4070\end{array}$ & $\begin{array}{l}0 \\
0 \\
0\end{array}$ & $\begin{array}{r}18.80 \\
4.80 \\
4.80\end{array}$ & $\begin{array}{l}1 \\
1 \\
1\end{array}$ & $\begin{array}{l}3.13 E-05 \\
3.74 E-06 \\
5.37 E-06\end{array}$ & $\begin{array}{l}3.13 \mathrm{E}-05 \\
3.74 \mathrm{E}-06 \\
5.37 \mathrm{E}-06\end{array}$ & $\begin{array}{l}\bar{D} \\
F \\
F\end{array}$ & $\begin{array}{l}9.80 \\
0.89 \\
2.65\end{array}$ \\
\hline
\end{tabular}


GXO Version 4.0

December 19, 1994

General Purpose Atmospheric Dispersion Code

Produced by Westinghouse Hanford Company

Users Guide documented in WHC-SD-GN-SWD-30002 ReV. 1.

Val idation documented in WHC-SD-GN-SWD-30003 ReV. 1.

code Custodian is: Brit E. Hey

West inghouse Hanford Company

P.O. Box 1970

$R$ ichland, WA 99352

(509) $376-2921$

Run Date $=03 / 13 / 96$

Run Time $=13: 53: 38.55$

INPUT ECHO:

CWC Solid Waste storage P4 to P7 Xes, case 6 .

c GXo Version 4.0 Input File

c mode

c

$$
1
$$

c MOOE CHOICE

$c$ mode $=1$ then $X / Q$ based on Hanford site specific meteorology

c mode $=2$ then $X / Q$ based on atmospheric stability $c$ lass and wind speed

$c$ mode $=3$ then $X / Q$ plot file is created

c

c LOGICAL CHOICES:

c ifox inorm icdf ichk isite ipop

$c$ ifox $=t$ then joint frequency used to compute frequency to exceed $x / Q$

$c \quad=f$ then joint frequency used to compute annual average $x / 0$

$c$ inorm $=t$ then joint frequency data is normalized (as in GENII)

c $=f$ then joint frequency data is un-normalized

c icdf = then cumlative distribution file created (CDF.OUT)

$c \quad=f$ then no cumulative distribution file created

$c$ ichk $=t$ then $X / Q$ parameter print option turned on

c $\quad=f$ then no parameter print

$c$ isite $=t$ then $X / Q$ based on joint frequency data for all 16 sectors

$c \quad=f$ then $X / Q$ based on joint frequency data of individual sectors

$c$ ipop $=t$ then $x / Q$ is population weighted

$c=f$ then no population weighting

$c$

C $X / Q$ AMD HIND SPEED ADJUSTMENT MODELS:

c ipuff idep isre iwind

$\begin{array}{llll}0 & 0 & 0 & t\end{array}$

c DifFUSION COEFFICIENT ADJUSTMENT MODELS:

C iwake ipm iflow ientr

c EFFECTIVE RELEASE HEIGHT ADJUSTMENT HODELS:

c (irise igrnd) iwash igrav

$\begin{array}{llll}1 & 1 & 0 & 0\end{array}$

$c$ ipuff $=1$ then $X / Q$ calculated using puff model

c $=0$ then $X / Q$ calculated using default continuous plume model

$c$ idep $=1$ then plume depletion model turned on (Chamberlain model)

$c$ isrc $=1$ then $X / Q$ multiplied by scalar

$c \quad=2$ then $X / Q$ adjusted by wind speed function

c iwind $=1$ then wind speed corrected for plume height

c iwake $=1$ then NRC RG 1.145 building wake model turned on

c $\quad=2$ then MACCS virtual distance building wake model turned on

c ipm $=1$ then MRC RG 1.145 plume meander model turned on

$c \quad=2$ then 5 th Power Las plume meander model turned on

c $\quad=3$ then sector average model turned on

c iflow $=1$ then sigmas adjusted for volume flow rate

$c$ ientr $=1$ then method of Pasquill used to account for entrainment

$c$ irise $=1$ then MACCs buoyant plume rise model turned on

c $\quad 2$ then ISC2 monentun/buayancy plume rise model turned on 


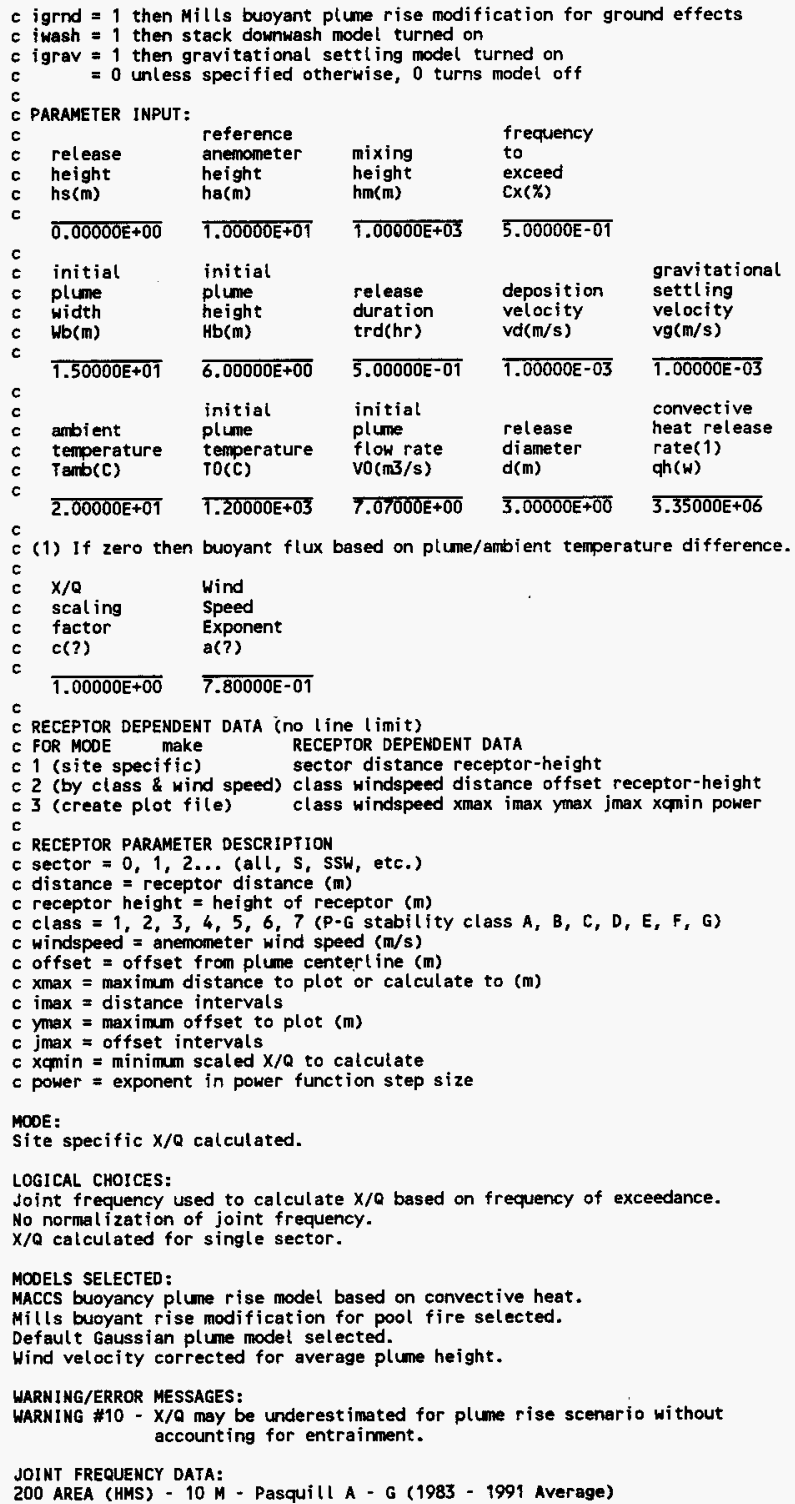


WHC-SD-WM-CN-016, Rev. 0

Created $8 / 26 / 92$ KR

CWC Solid Waste Storage P4 to P7 Xes, case 6 .

\begin{tabular}{|c|c|c|c|c|c|c|c|c|}
\hline ECTOR & $\begin{array}{c}\text { DISTANCE } \\
(\mathrm{m})\end{array}$ & $\begin{array}{c}\text { RECEPT } \\
\text { HE IGHT } \\
\text { (m) }\end{array}$ & $\begin{array}{l}\text { SECT. } \\
\text { FREQ. } \\
(\%)\end{array}$ & POPULATION & $\begin{array}{l}\text { TOTAL } \\
\text { POPULATION } \\
\text { SCALED } \\
X / 0 \\
(\mathrm{~s} / \mathrm{m} 3)\end{array}$ & $\begin{array}{l}\text { AVERAGE } \\
\text { IMDIVIDUAL } \\
\text { SCALED } \\
X / Q \\
(s / \mathrm{m3})\end{array}$ & $\begin{array}{l}\text { ATM. } \\
\text { STAB. } \\
\text { CLASS }\end{array}$ & $\begin{array}{l}\text { WIND } \\
\text { SPEED } \\
(\mathrm{m} / \mathrm{s})\end{array}$ \\
\hline $\begin{array}{l}\text { ESE } \\
W \\
W\end{array}$ & $\begin{array}{r}498 \\
11700 \\
4070\end{array}$ & c & $\begin{array}{r}18.80 \\
4.80 \\
4.80\end{array}$ & $\begin{array}{l}1 \\
1\end{array}$ & $\begin{array}{l}9.77 E-04 \\
4.36 E-06 \\
7.03 E-06\end{array}$ & $\begin{array}{l}9.77 \mathrm{E}-04 \\
4.36 \mathrm{E}-06 \\
7.03 \mathrm{E}-06\end{array}$ & $\begin{array}{l}\mathbf{G} \\
\mathbf{F} \\
\mathbf{F}\end{array}$ & $\begin{array}{l}4.70 \\
4.70 \\
2.65\end{array}$ \\
\hline
\end{tabular}


WHC-SD-WM-CN-016, Rev. 0

GXO Version 4.0

December 19, 1994

General Purpose Atmospheric Dispersion Code

Produced by Westinghouse Hanford Company

Users Guide documented in WHC-SD-GN-SWD-30002 Rev. 1.

Val idation documented in WHC-SD-GN-SWD-30003 Rev. 1.

Code Custodian is: Brit E. Hey

West inghouse Hanford Company

P.0. Box 1970

Richland, WA 99352

(509) $376-2921$

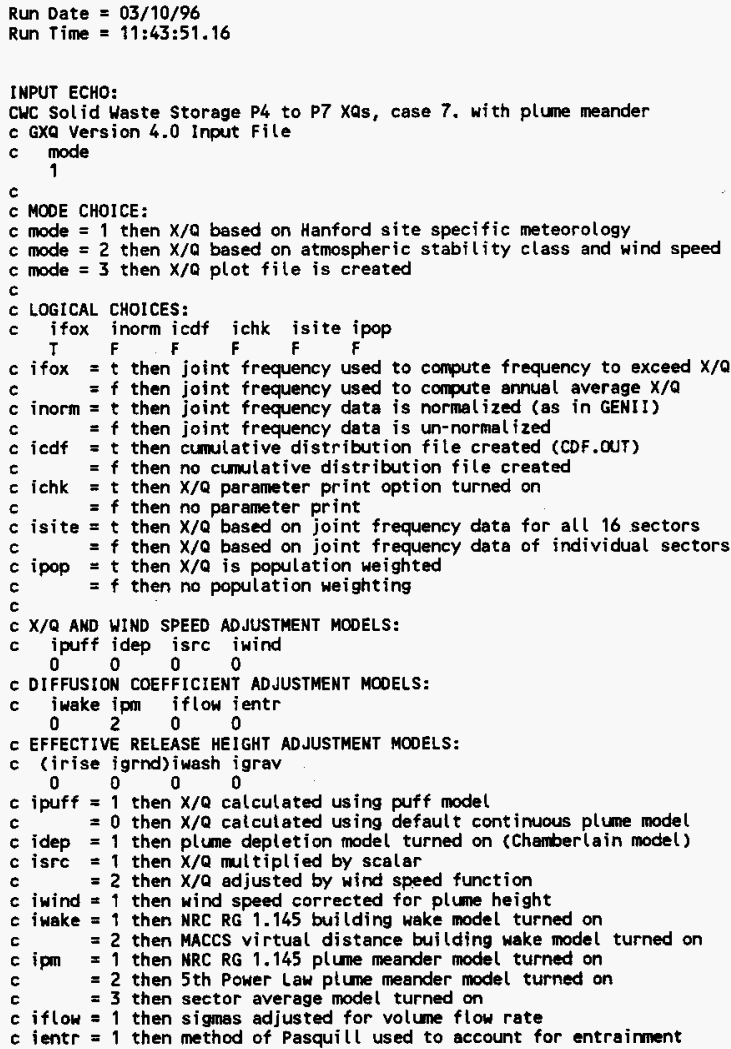




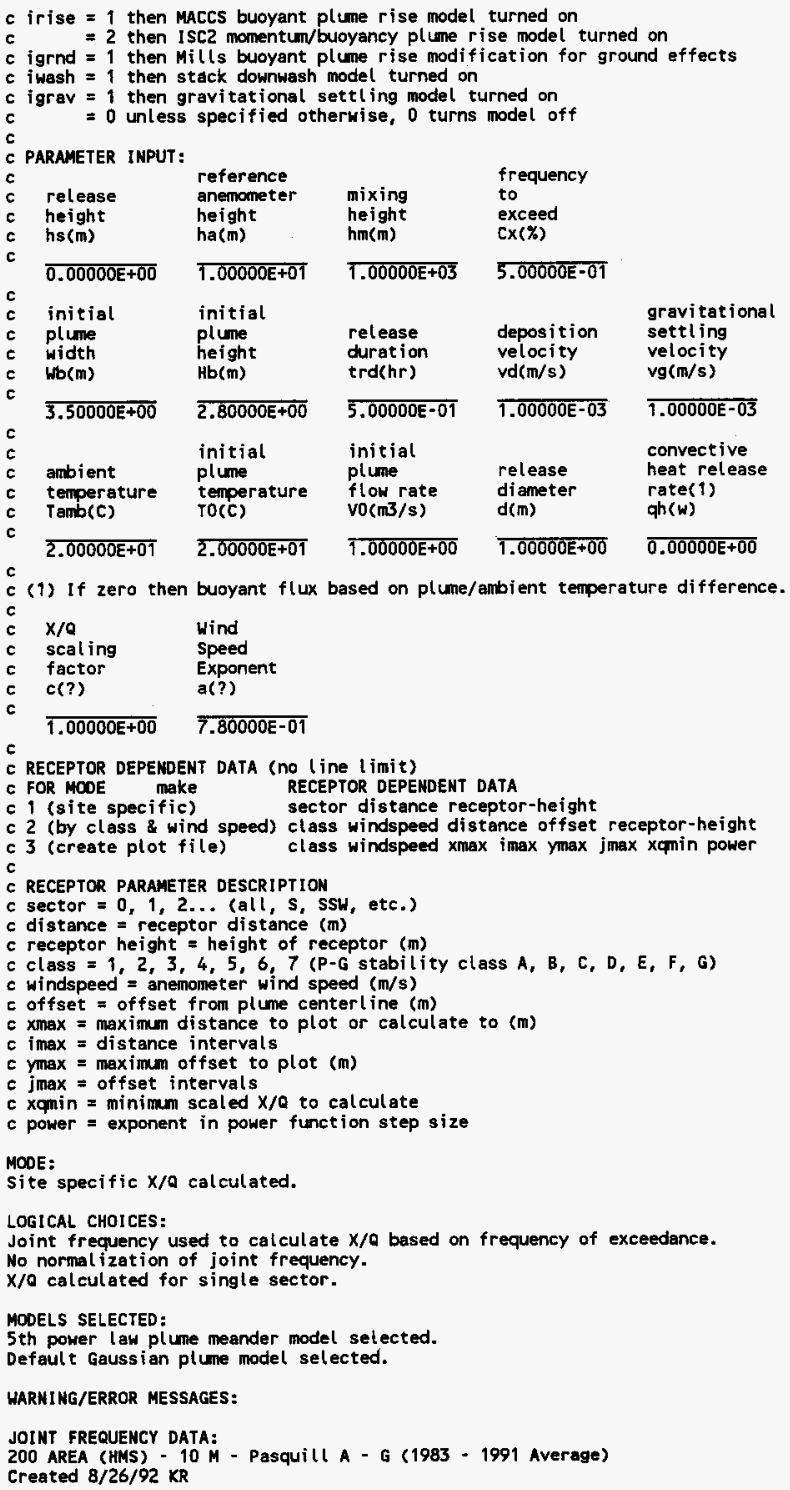


WHC-SD-WM-CN-016, Rev, 0

CWC Solid Waste storage P4 to P7 XQs, case 7. With plume meander

\begin{tabular}{|c|c|c|c|c|c|c|c|c|}
\hline SECTOR & $\begin{array}{l}\text { DISTANCE } \\
\text { (II) }\end{array}$ & $\begin{array}{c}\text { RECEPT } \\
\text { HEIGHT } \\
\text { (m) }\end{array}$ & $\begin{array}{l}\text { SECT. } \\
\text { FREQ. } \\
(\%)\end{array}$ & POPULATION & $\begin{array}{l}\text { TOTAL } \\
\text { POPULATION } \\
\text { SCALED } \\
X / a \\
(\mathrm{~s} / \mathrm{m} 3)\end{array}$ & $\begin{array}{l}\text { AVERAGE } \\
\text { INDIVIDUAL } \\
\text { SCALED } \\
X / 9 \\
(\mathrm{~s} / \mathrm{m} 3)\end{array}$ & $\begin{array}{l}\text { ATM. } \\
\text { STAB. } \\
\text { CLASS }\end{array}$ & $\begin{array}{l}\text { HIND } \\
\text { SPEED } \\
(\mathrm{m} / \mathrm{s})\end{array}$ \\
\hline SE & $\begin{array}{r}498 \\
11700 \\
4070\end{array}$ & 0 & $\begin{array}{r}18.80 \\
4.80 \\
4.80\end{array}$ & $\begin{array}{l}1 \\
1\end{array}$ & $\begin{array}{l}1.68 \mathrm{E}-03 \\
1.41 \mathrm{E}-05 \\
5.60 \mathrm{E}-05\end{array}$ & $\begin{array}{l}1.68 \mathrm{E}-03 \\
1.41 \mathrm{E}-05 \\
5.60 \mathrm{E}-05\end{array}$ & $\begin{array}{l}F \\
G \\
G\end{array}$ & $\begin{array}{l}0.89 \\
2.65 \\
2.65\end{array}$ \\
\hline
\end{tabular}


WHC-SD-WM-CN-016, Rev. 0

APPENDIX C

GENII INPUT AND OUTPUT FILES 
\#\#\#\#\#\#\#\#\#\#\#\#\#\#\#\#\#\#\#\#\# Program GENII Input File \#\#\#\#\#\#\#\#\#\# 8 Jul 88 \#\#\#\#

Title: CWC - MFP-percent by activity-soluble $\mathrm{Pu}$.

$\backslash$ GENI I $\backslash$ cwcmfp. in

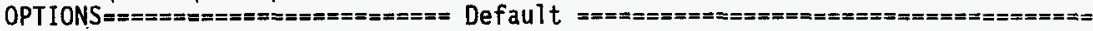

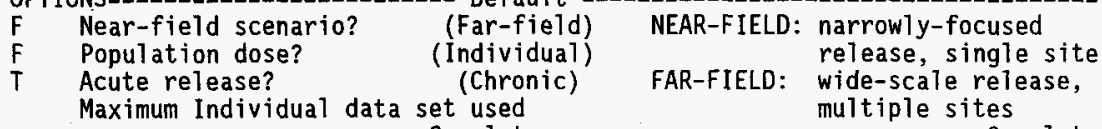

Complete

TRANSPORT OPTIONS $===== \pm======$ Section

$T$ Air Transport

EXPOSURE PATHWAY OPTIONS $=====$ Section

F Surface Water Transport

F Biotic Transport (near-field) 3,4

F Waste Form Degradation (near) 3,4

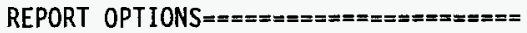

$T$ Report AEDE only

$T$ Report by radionuclide

$T$ Report by exposure pathway

$F$ Debug report on screen

$F$ Finite plume, external 5

$T$ Infinite plume, external 5

$F$ Ground, external

$F$ Recreation, external

T Inhalation uptake 5,6

$F$ Drinking water ingestion 7,8

$F$ Aquatic foods ingestion 7,8

$F$ Terrestrial foods ingestion 7,9

$F$ Animal product ingestion $.7,10$

$F$ Inadvertent soil ingestion

\section{INVENTORY \#\#\#\#\#\#\#\#\#\#\#\#\#\#\#\#\#\#\#\#\#\#\#\#\#\#\#\#\#\#\#\#\#\#\#\#\#\#\#\#\#\#\#\#\#\#\#\#\#\#\#\#\#\#\#\#\#\#\#\#\#\#\#\#\#\#\#\#}

4 Inventory input activity units: (1-pCi 2-uCi $3-\mathrm{mCl}^{4} 4-\mathrm{Ci}$ 5-Ba)

0 Surface soil source units (1- m2 $\left.2-\mathrm{m}_{3} 3-\mathrm{kg}\right)$

Equilibrium question goes here

\begin{tabular}{|c|c|c|c|c|c|c|c|c|}
\hline Use when & tr & $\begin{array}{l}\text { ease Tern } \\
\text { port sel }\end{array}$ & ected & & field sce & enaric & & \\
\hline $\begin{array}{l}\text { Release } \\
\text { Radio- } \\
\text { nuclide }\end{array}$ & $\begin{array}{l}\text { Air } \\
/ y r\end{array}$ & $\begin{array}{l}\text { Surface } \\
\text { Water } \\
\text { /yr }\end{array}$ & $\begin{array}{l}\text { Buried } \\
\text { Waste } \\
/ \mathrm{m}^{3}\end{array}$ & $\begin{array}{l}\text { Air } \\
\text { im3 }\end{array}$ & $\begin{array}{l}\text { Surface } \\
\text { Soil } \\
\text { /unit }\end{array}$ & $\begin{array}{l}\text { Deep } \\
\text { Soil } \\
/ m 3\end{array}$ & $\begin{array}{l}\text { Ground } \\
\text { Water } \\
\text { /L }\end{array}$ & $\begin{array}{l}\text { Surface } \\
\text { Water } \\
/ L\end{array}$ \\
\hline
\end{tabular}

CS137 2.5E-1

SR90 $\quad 1.9 \mathrm{E}-1$

Y $90 \quad 1.9 \mathrm{E}-1$

PU239 4.1E-3

PU241 1.0E-1

AM241 7.9E-3

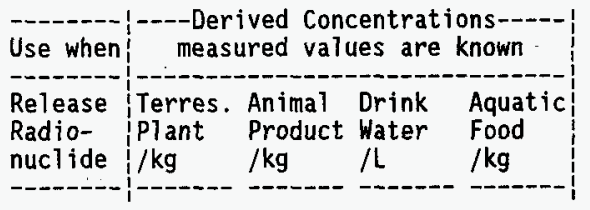

TIME

1 Intake ends after (yr)

50 Dose calc. ends after (yr)

0 Release ends after (yr) 
WHC-SD-WM-CN-016, Rev. 0

0 No. of years of air deposition prior to the intake period

0 No. of years of irrigation water deposition prior to the intake period

FAR-FIELD SCENARIOS (IF POPULATION DOSE) \#\#\#\#\#\#\#\#\#\#\#\#\#\#\#\#\#\#\#\#\#\#\#\#\#\#\#\#\#\#\#\#\#

0

0

NEAR-FIELD SCENARIOS \#\#\#\#\#\#\#\#\#\#\#\#\#\#\#\#\#\#\#\#\#\#\#\#\#\#\#\#\#\#\#\#\#\#\#\#\#\#\#\#\#\#\#\#\#\#\#\#\#\#\#\#\#

Prior to the beginning of the intake period: (yr)

When was the inventory disposed? (Package degradation starts)

When was LOIC? (Biotic transport starts)

Fraction of roots in upper soil (top $15 \mathrm{~cm}$ )

Fraction of roots in deep soil

Manual redistribution: deep soil/surface soil dilution factor

Source area for external dose modification factor (m2)

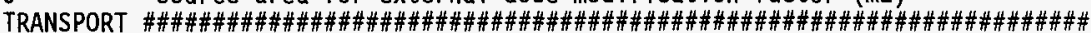

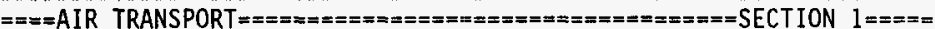
0 -Calculate PM

10

Option: l-Use chi/0 or PM value IF

2-Select MI dist \& dir 0

3-Specify MI dist \& dir 0

Chi/Q or PM value

MI sector index $(1=S)$

0

Release type $(0-3)$

Stack release (T/F)

Stack height (m)

Stack flow (m3/sec)

Stack radius (m)

EffTuent temp. (C)

Building $x$-section (m2)

Building height (m)

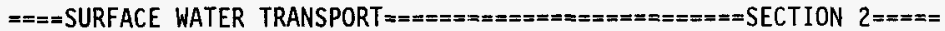
Mixing ratio model: 0-use value, 1-river, 2-lake

Mixing ratio, dimensionless

Average river flow rate for: $M I X F L G=0(\mathrm{~m} 3 / \mathrm{s}), \operatorname{MIXFLG}=1,2(\mathrm{~m} / \mathrm{s})$, Transit time to irrigation withdrawl location (hr)

If mixing ratio model $>0$ :

Rate of effluent discharge to receiving water body (m3/s)

Longshore distance from release point to usage location (m)

offshore distance to the water intake (m)

Average water depth in surface water body $(\mathrm{m})$

Average river width (m), MIXFLG $=1$ only

Depth of effluent discharge point to surface water (m), lake only

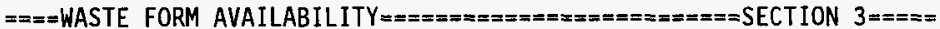
Waste form/package half life, $(y r)$

Waste thickness, (m)

Depth of soil overburden, $m$

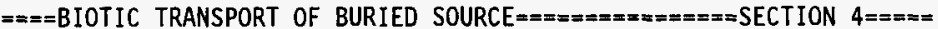
Consider during inventory decay/buildup period $(T / F)$ ?

Consider during intake period (T/F)? | 1-Arid non agricultural

Pre-Intake site condition............. 2-Humid non agricultural 3-Agricultural

EXPOSURE \#\#\#\#\#\#\#\#\#\#\#\#\#\#\#\#\#\#\#\#\#\#\#\#\#\#\#\#\#\#\#\#\#\#\#\#\#\#\#\#\#\#\#\#\#\#\#\#\#\#\#\#\#\#\#\#\#\#\#\#\#\#\#\#\#\#\#\#\# 
WHC-SD-WM-CN-016, Rev. 0

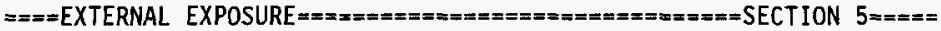
Exposure time:

Residential irrigation:

Plume (hr)

Soli contamination (hr)

Swimming (hr)

Boating (hr)

$T$

0

0

Shoreline activities (hr) 0

Shoreline type: (1-river, 2-1ake, 3-ocean, 4-tidal basin)

Transit time for release to reach aquatic recreation (hr)

Average fraction of time submersed in acute cloud ( $\mathrm{hr} /$ person $\mathrm{hr}$ )

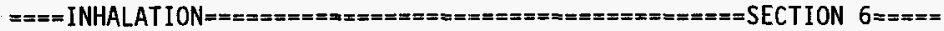
8766.0 Hours of exposure to contamination per year

0

0

0

0

0

0

$\mathrm{F}$

$\mathrm{F}$
0-No resus- 1-Use Mass Loading

pension Mass loading factor $(\mathrm{g} / \mathrm{m} 3)$ Top soil available $(\mathrm{cm})$

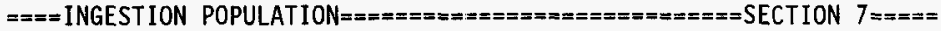
Atmospheric production definition (select option):

0 -Use food-weighted chi/Q, (food-sec/m3), enter value on this line 1-Use population-weighted chi/Q

2-Use uniform production

3-Use chi/Q and production grids (PRODUCTION will be overridden)

Population ingesting aquatic foods, 0 defaults to total (person)

Population ingesting drinking water, 0 defaults to total (person)

Consider dose from food exported out of region (default=F)

Note below: $S^{*}$ or Source: 0-none, 1-ground water, 2-surface water 3-Derived concentration entered above

$====$ AQUATIC FOODS / DRINKING WATER INGESTION=========SECTION $8====$

Salt water? (default is fresh)

\begin{tabular}{|c|c|c|c|c|c|c|c|}
\hline $\begin{array}{l}\text { USE } \\
? \\
\dot{T} / F\end{array}$ & $\begin{array}{l}\text { FOOD } \\
\text { TYPE }\end{array}$ & $\begin{array}{l}\text { TRAN- } \\
\text { SIT } \\
\text { hr }\end{array}$ & $\begin{array}{l}\text { PROD- } \\
\text { UCTION } \\
\mathrm{kg} / \mathrm{yr}\end{array}$ & $\begin{array}{l}\text { - CONSUM } \\
\text { HOLDUP } \\
\text { da }\end{array}$ & $\begin{array}{l}\text { TION- } \\
\text { RATE } \\
\mathrm{kg} / \mathrm{yr}\end{array}$ & & RINKING WATER \\
\hline $\begin{array}{l}F \\
F \\
F\end{array}$ & $\begin{array}{l}\text { FISH } \\
\text { MOLLUS } \\
\text { CRUSTA } \\
\text { PLANTS }\end{array}$ & $\begin{array}{l}0.00 \\
0.00 \\
0.00 \\
0.00\end{array}$ & $\begin{array}{l}0.0 \mathrm{E}+00 \\
0.0 \mathrm{E}+00 \\
0.0 \mathrm{E}+00 \\
0.0 \mathrm{E}+00\end{array}$ & $\begin{array}{l}0.00 \\
0.00 \\
0.00 \\
0.00\end{array}$ & $\begin{array}{l}0.0 \\
0.0 \\
0.0 \\
0.0\end{array}$ & $\begin{array}{l}0 \\
\mathrm{~T} \\
0 \\
0\end{array}$ & $\begin{array}{l}\text { Source (see above) } \\
\text { Treatment? T/F } \\
\text { Hldup/trans it (da) } \\
\text { Consumpton (L/yr) }\end{array}$ \\
\hline
\end{tabular}

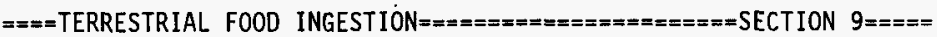

\begin{tabular}{|c|c|c|c|c|c|c|c|c|}
\hline $\begin{array}{l}\text { USE } \\
? \\
\text { T/F }\end{array}$ & $\begin{array}{l}\text { FOOD } \\
\text { TYPE }\end{array}$ & $\begin{array}{l}\text { GROW } \\
\text { TIME } \\
\text { da }\end{array}$ & $\begin{array}{l}\text {--IRRIGA } \\
\text { S RATE } \\
\text { * in/yr }\end{array}$ & $\begin{array}{l}\text { TION-- } \\
\text { TIME } \\
\text { mo/yr }\end{array}$ & $\begin{array}{l}\text { YIELD } \\
\mathrm{kg} / \mathrm{m} 2\end{array}$ & $\begin{array}{l}\text { PROD- } \\
\text { UCTION } \\
\mathrm{kg} / \mathrm{yr}\end{array}$ & $\begin{array}{l}-- \text { CONSL } \\
\text { HOLDUP } \\
\mathrm{da}\end{array}$ & $\begin{array}{l}\text { TION-- } \\
\text { RATE } \\
\mathrm{kg} / \mathrm{yr}\end{array}$ \\
\hline $\begin{array}{l}F \\
F \\
F\end{array}$ & $\begin{array}{l}\text { LEAF V } \\
\text { ROOT } V \\
\text { FRUIT } \\
\text { GRAIN }\end{array}$ & $\begin{array}{l}0.00 \\
0.00 \\
0.00 \\
0.00\end{array}$ & $\begin{array}{l}0.0 \\
0.0 \\
0.0 \\
0.0\end{array}$ & $\begin{array}{l}0.0 \\
0.0 \\
0.0 \\
0.0\end{array}$ & $\begin{array}{l}0.0 \\
0.0 \\
0.0 \\
0.0\end{array}$ & $\begin{array}{l}0.0 \mathrm{E}+00 \\
0.0 \mathrm{E}+00 \\
0.0 \mathrm{E}+00 \\
0.0 \mathrm{E}+00\end{array}$ & $\begin{array}{l}0.0 \\
0.0 \\
0.0 \\
0.0\end{array}$ & $\begin{array}{l}0.0 \\
0.0 \\
0.0 \\
0.0\end{array}$ \\
\hline
\end{tabular}

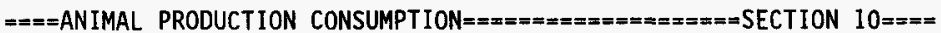


WHC-SD-WM-CN-016, Rev. 0

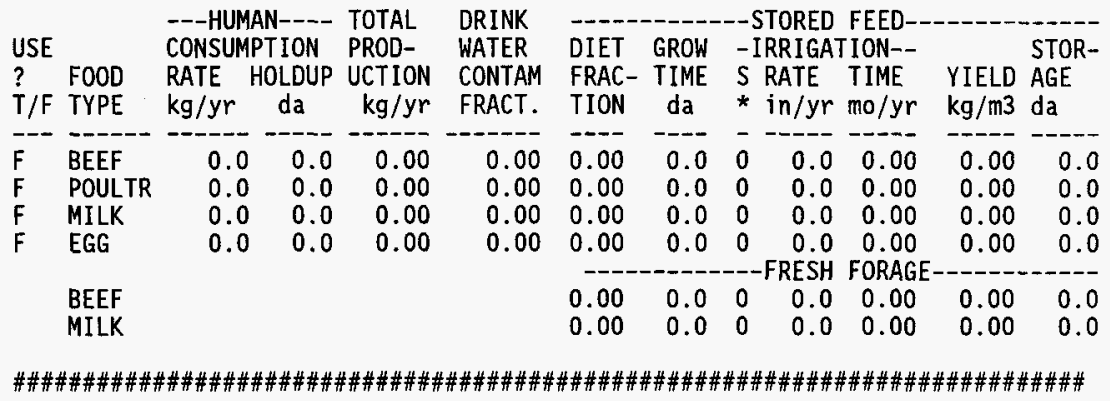


Case title: CWC - MFP-percent by activity-soiuble Pu. 1

Executed on: $02 / 28 / 96$ at $09: 51: 59$

Page A.

This is a far-field (wide-scale release, multiple site) scenario. Release is acute

Individual dose

THE FOLLOWING TRANSPORT MODES ARE CONSIDERED

Air

THE FOLLOWING EXPOSURE PATHS ARE CONSIDERED:

Infinite plume, external

Inhalation uptake

THE FOLLOWING TIMES ARE USED:

Intake ends after (yr): $\quad 1.0$

Dose calculations ends after $(y r): \quad 50.0$

$======== \pm=$ FILENAMES AND TITLES OF FILES/LIBRARIES USED

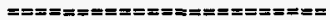

Input fiTe name: \GENII\CWCmfp. in

GENII Default Parameter Values (28-Mar-90 RAP)

Radionuclide Library - Times $<100$ years (23-July-93 PDR)

External Dose Factors for GENII in person Sv/yr per Bq/n (8-May-90 R

Worst-Case Solubilities, Yearly Dose Increments (23-Ju1-93 PDR)

\begin{tabular}{|c|c|c|c|}
\hline $\begin{array}{l}\text { Release } \\
\text { Radio- } \\
\text { nucl ide }\end{array}$ & $\underset{\mathrm{Ci} / \mathrm{yr}}{\mathrm{Air}}$ & $\begin{array}{c}\text { Surface } \\
\text { Water } \\
\mathrm{Ci} / \mathrm{yr}\end{array}$ & $\begin{array}{l}\text { Buried } \\
\text { Source } \\
\mathrm{Ci} / \mathrm{m} 3\end{array}$ \\
\hline $\begin{array}{l}\text { CS137 } \\
\text { SR90 } \\
\text { Y } 90 \\
\text { PU239 }\end{array}$ & $\begin{array}{l}2.5 \mathrm{E}-01 \\
1.9 \mathrm{E}-01 \\
1.9 \mathrm{E}-01 \\
4.1 \mathrm{E}-03\end{array}$ & $\begin{array}{l}0.0 E+00 \\
0.0 E+00 \\
0.0 E+00 \\
0.0 E+00\end{array}$ & $\begin{array}{l}0.0 E+00 \\
0.0 E+00 \\
0.0 E+00 \\
0.0 E+00\end{array}$ \\
\hline
\end{tabular}


WHC-SD-WM-CN-016, Rev. 0

PU241 $1.0 \mathrm{E}-01 \quad 0.0 \mathrm{E}+00 \quad 0.0 \mathrm{E}+00$

AM241 7.9E-03 $0.0 \mathrm{E}+00 \quad 0.0 \mathrm{E}+00$

$==========$ AIR TRANSPORT

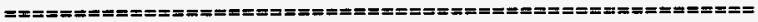

$1.0 E+00$ Input $E / Q$ value $(s / m 3)$

$=========$ EXTERNAL EXPOSURE

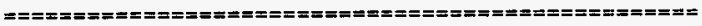

$1.0 \mathrm{E}+00$ Fraction of time spent in cloud

$===== \pm==x=$ INHALATION

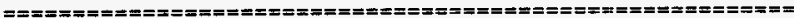

Resuspension not considered

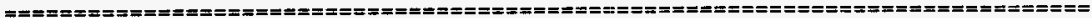

$=$

Input prepared by:

Date:

Input checked by:

Date:

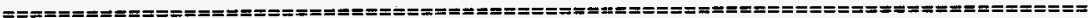

$=$ 
Case title: CWC - MFP-percent by activity-soluble Pu.

Executed on: $02 / 28 / 96$ at $09: 52: 11$

Page $C$. 1

Acute release

Uptake/exposure period:

Dose commitment period:

Dose units:

1.0

Rem 50.0

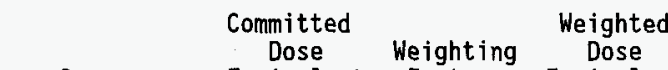

$\begin{array}{cccc}\text { Organ } & \text { Equivalent } & \text { Factors Equivalent } \\ \text { Gonads } & 5.3 \mathrm{E}+02 & 2.5 \mathrm{E}-01 & 1.3 \mathrm{E}+02\end{array}$

Breast $2.6 \mathrm{E}+00 \quad 1.5 \mathrm{E}-01 \quad 3.9 \mathrm{E}-0 \mathrm{I}$

R Marrow $3.0 \mathrm{E}+03 \quad 1.2 \mathrm{E}-01 \quad 3.6 \mathrm{E}+02$

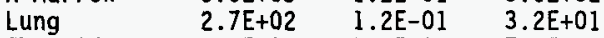

Thyroid $2.6 \mathrm{E}+00 \quad 3.0 \mathrm{E}-02 \quad 7.7 \mathrm{E}-02$

Bone Sur $3.8 \mathrm{E}+04 \quad 3.0 \mathrm{E}-02 \quad 1.1 \mathrm{E}+03$

Liver $\quad 6.5 \mathrm{E}+03 \quad 6.0 \mathrm{E}-02.3 .9 \mathrm{E}+02$

LL Int. $\quad 7.0 \mathrm{E}+00 \quad 6.0 \mathrm{E}-02 \quad 4.2 \mathrm{E}-01$

UL Int. $\quad 4.4 \mathrm{E}+00 \quad 6.0 \mathrm{E}-02 \quad 2.6 \mathrm{E}-01$

S Int. $\quad 3.2 \mathrm{E}+00 \quad 6.0 \mathrm{E}-02 \quad 1.9 \mathrm{E}-01$

Stomach $\quad 2.9 \mathrm{E}+00 \quad 6.0 \mathrm{E}-02 \quad 1.7 \mathrm{E}-01$

Internal Effective Dose Equivalent 2.0E +03

External Dose 2.9E-02

Annual Effective Dose Equivalent 2.OE+03 
WHC-SD-WM-CN-016, Rev. 0

Total Inhalation EDE:

2. $0 \mathrm{E}+03$

Total Ingestion EDE:

$0.0 E+00$ 
WHC-SD-WM-CN-016, Rev. 0

GENII Dose Calculation Program

(Version 1.485 3-Dec-90)

Case title: CWC - MFP-percent by activity-soluble Pu.

Executed on: $02 / 28 / 96$ at $09: 52: 11$

Page $C$. 2

Acute release

Uptake/exposure period:

Dose commitment period:

Dose units:

1.0

Rem

50.0

Dose Commitment Year

12

$3 \quad \ldots$

Internal : I

Intake : !

Year: 3

$0.0 \mathrm{E}+00 \quad \ldots$

i

$+$

21

$0.0 \mathrm{E}+00$

$0.0 \mathrm{E}+00$

Interna]

I

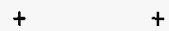

Effective

$1: 8$.

$8.3 E+0$

$+5.5 E+0$

$+5.4 \mathrm{E}+0$

Dose

Equivalent

II II II

Internal

Cumulative

Annua 1

$8.3 E+01+5.5 E+01+5.4 E+01+\ldots$

$=2.0 \mathrm{E}+03$

Interna1

Dose

Dose

Externa1

$+\quad+\quad+$


WHC-SD-WM-CN-016, Rev. 0

$\begin{array}{llllll}\text { Annual } & 2.9 \mathrm{E}-02 & 0.0 \mathrm{E}+00 & 0.0 \mathrm{E}+00 & \ldots & 2.9 \mathrm{E}-02\end{array}$

Dose

Annua 1

i! $11 \quad$ in

Dose

$8.3 E+01+5.5 E+01+5.4 E+01+\ldots=2.0 E+03$ Dose

Maximum

8.3E+01 Annual

Dose Occurred

In Year 1 
Case title: CWC - MFP-percent by activity-soluble Pu. 3

Executed on: $02 / 28 / 96$ at $09: 52: 11$

Page $C$.

Acute release

Uptake/exposure period:

Dose commitment period:

Dose units:

$$
\operatorname{Rem}^{1.0} \begin{array}{r}
50.0 \\
\end{array}
$$

Committed Dose Equivalent, by Exposure Pathway

Pathway Lung Stomach S Int. Ul. Int. LL Int. Bone Su R Marro Testes

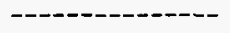

- -----

Inhale

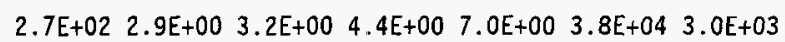

5. $3 \mathrm{E}+02$

Total

5. $3 \mathrm{E}+02$

$2.7 \mathrm{E}+02 \quad 2.9 \mathrm{E}+00 \quad 3.2 \mathrm{E}+00 \quad 4.4 \mathrm{E}+00 \quad 7.0 \mathrm{E}+00 \quad 3.8 \mathrm{E}+04 \quad 3.0 \mathrm{E}+03$

\begin{tabular}{l} 
Pathway \\
Inhale \\
\hline Total
\end{tabular}

Ovaries Muscle Thyroid Liver

-

$5.2 E+02 \quad 2.6 E+00 \quad 2.6 E+00 \quad 6.5 E+03$

Total

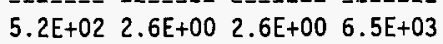

External Dose by Exposure Pathway

\begin{tabular}{ll} 
Pathway & \\
\hline Plume & $2.9 \mathrm{E}-02$ \\
\hline Total & $-9 .-9 \mathrm{E}-02$
\end{tabular}


Case title: CWC - MFP-percent by activity-soluble Pu. 4

Executed on: $02 / 28 / 96$ at $09: 52: 11$

Page $C$.

Acute release

Uptake/exposure period:

Dose commitment period:

Dose units:

1.0

50.0

Committed Dose Equivalent by Radionuclide

Radionuclide Lung Stomach S Int. UL Int. LL Int. Bone Su R Marro Testes

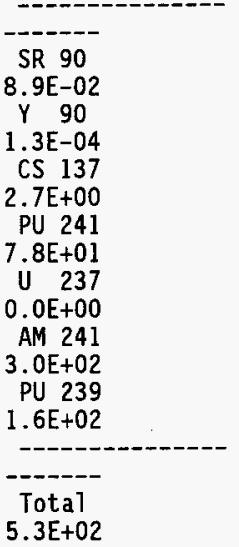

3.5E-01 9.6E-02 1.0E-01 2.3E-01 7.2E-01 $1.5 \mathrm{E}+02 \quad 6.8 \mathrm{E}+01$

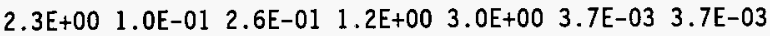

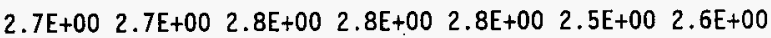

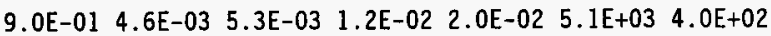

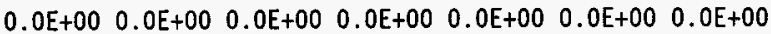

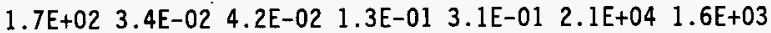

$\begin{array}{lllll}8.8 \mathrm{E}+01 & 7.8 \mathrm{E}-03 & 1.2 \mathrm{E}-02 & 5.0 \mathrm{E}-02 & 1.5 \mathrm{E}-01 \quad 1.1 \mathrm{E}+04 \quad 8.7 \mathrm{E}+02\end{array}$

$2.7 E+02 \quad 2.9 E+00 \quad 3.2 E+00 \quad 4.4 E+00 \quad 7.0 E+00 \quad 3.8 E+04 \quad 3.0 E+03$

5. $3 \mathrm{E}+02$

\begin{tabular}{lllll} 
Radionuclide & Ovaries & Muscle & Thyroid Liver \\
\hdashline SR 90 & $8.9 \mathrm{E}-02$ & $8.6 \mathrm{E}-02$ & $8.9 \mathrm{E}-02$ & $0.0 \mathrm{E}+00$ \\
Y 90 & $1.2 \mathrm{E}-04$ & $1.2 \mathrm{E}-04$ & $1.2 \mathrm{E}-04$ & $3.7 \mathrm{E}-03$ \\
CS 137 & $2.5 \mathrm{E}+00$ & $2.4 \mathrm{E}+00$ & $2.4 \mathrm{E}+00$ & $0.0 \mathrm{E}+00$ \\
PU 241 & $7.6 \mathrm{E}+01$ & $4.9 \mathrm{E}-03$ & $2.1 \mathrm{E}-03$ & $8.0 \mathrm{E}+02$ \\
U 237 & $0.0 \mathrm{E}+00$ & $0.0 \mathrm{E}+00$ & $0.0 \mathrm{E}+00$ & $0.0 \mathrm{E}+00$ \\
AM 241 & $2.9 \mathrm{E}+02$ & $2.9 \mathrm{E}-02$ & $1.6 \mathrm{E}-02$ & $3.8 \mathrm{E}+03$ \\
PU 239 & $1.5 \mathrm{E}+02$ & $4.8 \mathrm{E}-03$ & $4.7 \mathrm{E}-03$ & $2.0 \mathrm{E}+03$
\end{tabular}


WHC-SD-WM-CN-016, Rev. 0

\section{Total}

$5.2 E+02 \quad 2.6 E+00 \quad 2.6 E+00 \quad 6.5 E+03$ 


\section{GENII Dose Calculation Program \\ (Version 1.485 3-Dec-90)}

Case title: CWC - MFP-percent by activity-soluble Pu. 5

Executed on: $02 / 28 / 96$ at $09: 52: 11$

Page $C$.

Acute release

Uptake/exposure period:

Dose commitment period:

Dose units:
1.0

50.0

Rem

\begin{tabular}{|c|c|c|}
\hline $\begin{array}{l}\text { Radio- } \\
\text { nuclide }\end{array}$ & $\begin{array}{c}\text { Inhalation } \\
\text { Effective } \\
\text { Dose } \\
\text { Equivalent }\end{array}$ & $\begin{array}{l}\text { Ingestion } \\
\text { Effective } \\
\text { Dose } \\
\text { Equivalent }\end{array}$ \\
\hline----1 & $--m-1-0-1$ & - \\
\hline $\begin{array}{ll}\text { SR } & 90 \\
Y & 90 \\
\text { CS } & 137 \\
\text { PU } & 241 \\
\text { U } & 237 \\
\text { AM } & 241 \\
\text { PU } & 239\end{array}$ & $\begin{array}{l}1.3 \mathrm{E}+01 \\
5.5 \mathrm{E}-01 \\
2.5 \mathrm{E}+00 \\
2.7 \mathrm{E}+02 \\
0.0 \mathrm{E}+00 \\
1.2 \mathrm{E}+03 \\
6.1 \mathrm{E}+02\end{array}$ & $\begin{array}{l}0.0 \mathrm{E}+00 \\
0.0 \mathrm{E}+00 \\
0.0 \mathrm{E}+00 \\
0.0 \mathrm{E}+00 \\
0.0 \mathrm{E}+00 \\
0.0 \mathrm{E}+00 \\
0.0 \mathrm{E}+00\end{array}$ \\
\hline
\end{tabular}

\begin{tabular}{c} 
External \\
Dose \\
\hline $7.8 \mathrm{E}-06$ \\
$2.8 \mathrm{E}-04$ \\
$2.9 \mathrm{E}-02$ \\
$7.7 \mathrm{E}-12$ \\
$0.0 \mathrm{E}+00$ \\
$1.7 \mathrm{E}-05$ \\
$8.8 \mathrm{E}-08$ \\
\hline
\end{tabular}

\section{Internal} Effective Dose Equivalent

-

1. $3 \mathrm{E}+01$
5. $5 \mathrm{E}-01$

2. $5 \mathrm{E}+00$

2.7E+02

$0.0 \mathrm{E}+00$

1. $2 \mathrm{E}+03$

6. $1 E+02$
Annual

Effective Dose

Equivalent

$$
\text { 1. } 3 \mathrm{E}+01
$$

$5.5 \mathrm{E}-01$

2. $5 \mathrm{E}+00$

$2.7 \mathrm{E}+02$

$0.0 E+00$

1. $2 \mathrm{E}+03$

$6.1 \mathrm{E}+02$ 
\#\#\#\#\#\#\#\#\#\#\#\#\#\#\#\#\#\#\#\#\# Program GENII Input File \#\#\#\#\#\#\#\#\#\#\#8 Jul 88 \#\#\#\#

Title: CWC - MFP-percent by activity-insoluble $\mathrm{Pu}$. $\backslash$ GENI I \CWCmfpb. in

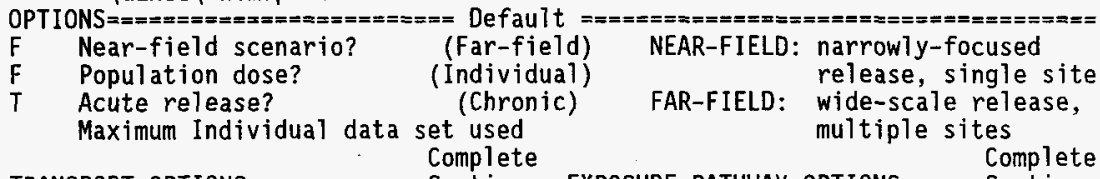

TRANSPORT OPTIONS===========: Section

$T$ Air Transport

F Surface Water Transport

F Biotic Transport (near-field) 3,4

$F$ Waste Form Degradation (near) 3,4

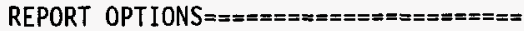

$T$ Report AEDE only

$T$ Report by radionuclide

$T$ Report by exposure pathway

$F$ Debug report on screen

EXPOSURE PATHWAY OPTIONS $=====$ Section

INVENTORY \#\#\#\#\#\#\#\#\#\#\#\#\#\#\#\#\#\#\#\#\#\#\#\#\#\#\#\#\#\#\#\#\#\#\#\#\#\#\#\#\#\#\#\#\#\#\#\#\#\#\#\#\#\#\#\#\#\#\#\#\#\#\#\#\#\#\#\#

4 Inventory input activity units: (1-pCi 2-uCi 3-mCi 4-Ci 5-Bq)

0 Surface soil source units (1- m2 $\left.2-\mathrm{m}^{3} 3-\mathrm{kg}\right)$

Equilibrium question goes here

\begin{tabular}{|c|c|c|c|c|c|c|c|c|}
\hline Use when & $t$ & $\begin{array}{l}\text { ase Ter } \\
\text { ort sel }\end{array}$ & ected & 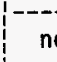 & $\begin{array}{l}-- \text { Basic } \\
\text { field sce }\end{array}$ & enaric & optiona & \\
\hline $\begin{array}{l}\text { Release } \\
\text { Radio- } \\
\text { nuclide }\end{array}$ & $\begin{array}{l}\text { Air } \\
/ y r\end{array}$ & $\begin{array}{l}\text { Surface } \\
\text { Water } \\
\text { /yr }\end{array}$ & $\begin{array}{l}\text { Buried } \\
\text { Waste } \\
\text { /m3 }\end{array}$ & $\begin{array}{l}\text { Air } \\
/ \mathrm{m} 3\end{array}$ & $\begin{array}{l}\text { Surface } \\
\text { Soil } \\
\text { /unit }\end{array}$ & $\begin{array}{l}\text { Deep } \\
\text { Soil } \\
/ \mathrm{m} 3\end{array}$ & $\begin{array}{l}\text { Ground } \\
\text { Water } \\
\text { /L }\end{array}$ & $\begin{array}{l}\text { Surface } \\
\text { Water } \\
\text { /L }\end{array}$ \\
\hline
\end{tabular}

SR90 1.9E-1

Y $90 \quad 1.9 \mathrm{E}-1$

PU239 4.1E-3

PU241 1.0E-1

AM241 $7.9 \mathrm{E}-3$

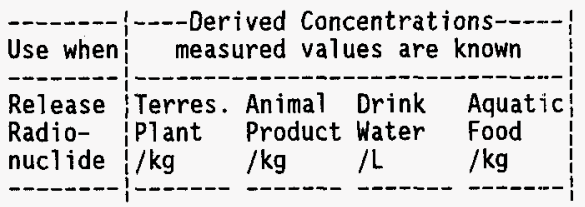

TIME \#\#\#\#\#\#\#\#\#\#\#\#\#\#\#\#\#\#\#\#\#\#\#\#\#\#\#\#\#\#\#\#\#\#\#\#\#\#\#\#\#\#\#\#\#\#\#\#\#\#\#\#\#\#\#\#\#\#\#\#\#\#\#\#\#\#\#\#\#\#\#

1 Intake ends after (yr)

50 Dose calc. ends after (yr)

0 Release ends after (yr) 
WHC-SD-WM-CN-016, Rev, 0

No. of years of air deposition prior to the intake period

0 No. of years of irrigation water deposition prior to the intake period

\section{FAR-FIELD SCENARIOS (IF POPULATION DOSE) \#\#\#\#\#\#\#\#\#\#\#\#\#\#\#\#\#\#\#\#\#\#\#\#\#\#\#\#\#\#\#\#}

0

0

\section{NEAR-FIELD SCENARIOS \#\#\#\#\#\#\#\#\#\#\#\#\#\#\#\#\#\#\#\#\#\#\#\#\#\#\#\#\#\#\#\#\#\#\#\#\#\#\#\#\#\#\#\#\#\#\#\#\#\#\#\#\#}

Prior to the beginning of the intake period: $(y r)$

0

0

0

0

0

0

TRANSPORT

0

0

0

$\mathrm{T}$

$\mathrm{T}$

0

\section{Definition option: 1-Use population grid in file POP. IN} 2-Use total entered on this line

Prior to the beginning of the intake period: (yr)
When was the inventory disposed? (Package degradation starts)
When was LOIC? (Biotic transport starts)
Fraction of roots in upper soil (top $15 \mathrm{~cm}$ )
Fraction of roots in deep soil
Manual redistribution: deep soil/surface soil dilution factor
0
Source area for external dose modificat ion factor (m2)
0
0

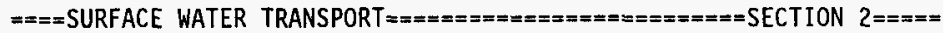
Mixing ratio mode1: 0-use value, 1-river, 2-lake

Mixing ratio, dimensionless

Average river flow rate for: $M I X F L G=0(\mathrm{~m} 3 / \mathrm{s}), \operatorname{MIXFLG}=1,2(\mathrm{~m} / \mathrm{s})$, Transit time to irrigation withdrawl location (hr).

If mixing ratio model $>0$ :

Rate of effluent discharge to receiving water body (m3/s)

Longshore distance from release point to usage location (m)

Offshore distance to the water intake $(m)$

Average water depth in surface water body $(\mathrm{m})$

Average river width (m), MIXFLG=I only

Depth of effluent discharge point to surface water (m), lake only

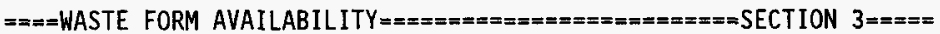
Waste form/package half life, (yr)

Waste thickness, (m)

Depth of soil overburden, $m$

$====$ BIOTIC TRANSPORT OF BURIED SOURCE $====m==m=m=====$ SECTION $4=====$ Consider during inventory decay/buildup period (T/F)?

Consider during intake period (T/F)? I-Arid non agricultural

Pre-Intake site condition............ 2-Humid non agricultural 
WHC-SD-WM-CN-016, Rev. 0

$====$ EXTERNAL EXPOSURE

Exposure time:

Plume (hr)

0

0

0

0

0

0

1.0

8766.0

0

0

0

0

0

0

$\mathrm{F}$

F
Soil contamination (hr)

Swimming (hr)

Boating (hr)

Shoreline activities (hr)

$=$ SECTION $5=====$

Residential irrigation:

Consider: (T/F)

Source: 1-ground water

2-surface water

Application rate (in/yr)

Duration (mo/yr)

Shorel ine type: (1-river, 2-lake, 3-ocean, 4-tidal basin)

Transit time for release to reach aquatic recreation (hr)

Average fraction of time submersed in acute cloud (hr/person hr)

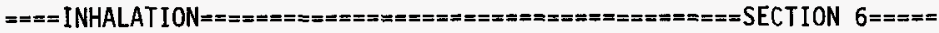

Hours of exposure to contamination per year

0-No resus- 1-Use Mass Loading 2-Use Anspaugh model

pension Mass loading factor $(\mathrm{g} / \mathrm{m} 3)$ Top soil available (cm)

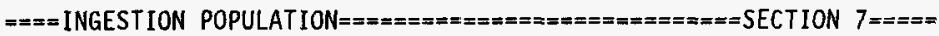

Atmospheric production definition (select option):

0 -Use food-weighted $\operatorname{chi} / Q,($ food $-\mathrm{sec} / \mathrm{m} 3)$, enter value on this 1 ine $1-$ Use-population-weighted chi/Q

2-Use uniform production

3-Use chi/Q and production grids (PRODUCTION will be overridden)

Population ingesting aquatic foods, 0 defaults to total (person)

Population ingesting drinking water, 0 defaults to total (person)

Consider dose from food exported out of region (default $t=F$ )

Note below: $S^{*}$ or Source: 0 -none, 1-ground water, 2-surface water 3-Derived concentration entered above

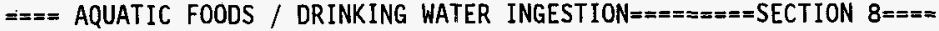

Salt water? (default is fresh)

\begin{tabular}{|c|c|c|c|c|c|}
\hline $\begin{array}{l}\text { USE } \\
? \stackrel{\text { FOOD }}{\text { T/F TYPE }}\end{array}$ & $\begin{array}{l}\text { TRAN- } \\
\text { SIT } \\
\mathrm{hr}\end{array}$ & $\begin{array}{l}\text { PROD- } \\
\text { UCTION } \\
\mathrm{kg} / \mathrm{yr}\end{array}$ & $\begin{array}{l}\text {-CONSUMF } \\
\text { HOLDUP } \\
\text { da }\end{array}$ & $\begin{array}{l}\text { PTION- } \\
\text { RATE } \\
\mathrm{kg} / \mathrm{yr}\end{array}$ & DRINKING WATER \\
\hline $\begin{array}{l}\text { FISH } \\
\text { MOLLUS } \\
\text { CRUSTA } \\
\text { PLANTS }\end{array}$ & $\begin{array}{l}0.00 \\
0.00 \\
0.00 \\
0.00\end{array}$ & $\begin{array}{l}0.0 \mathrm{E}+00 \\
0.0 \mathrm{E}+00 \\
0.0 \mathrm{E}+00 \\
0.0 \mathrm{E}+00\end{array}$ & $\begin{array}{l}0.00 \\
0.00 \\
0.00 \\
0.00\end{array}$ & $\begin{array}{l}0.0 \\
0.0 \\
0.0 \\
0.0\end{array}$ & $\begin{array}{l}\text { Source (see above) } \\
\text { Treatment? T/F } \\
\text { Hldup/transit (da) } \\
\text { Consumpton (L/yr) }\end{array}$ \\
\hline
\end{tabular}

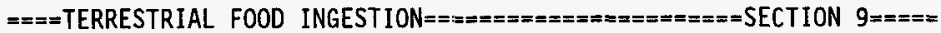

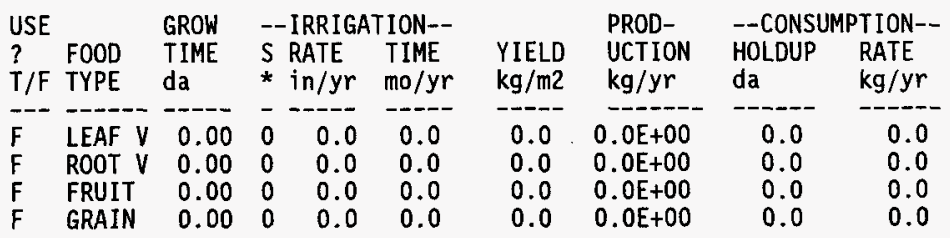

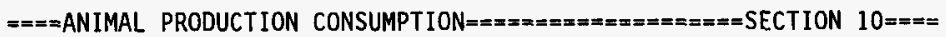


WHC-SD-WM-CN-016, Rev. 0

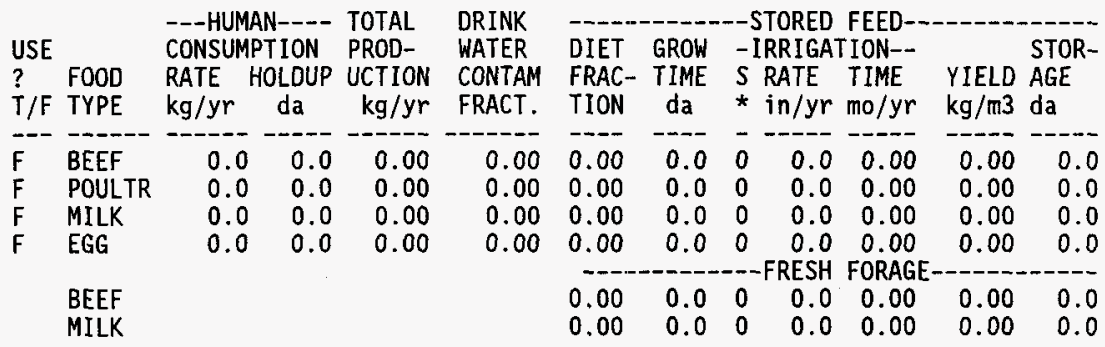

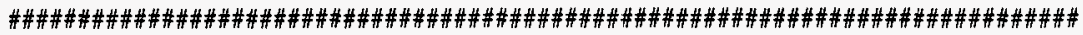




\section{GENII Dose Calculation Program}

(Version 1.485 3-Dec-90)

Case titie: CWC - MFP-percent by activity-insoluble Pu. 1

Executed on: $02 / 28 / 96$ at $09: 58: 12$

Page A.

This is a far-field (wide-scale release, multiple site) scenario.

Release is acute

Individual dose

THE FOLLOWING TRANSPORT MODES ARE. CONSIDERED Air

THE FOLLOWING EXPOSURE PATHS ARE CONSIDERED:

Infinite plume, external

Inhalation uptake

THE FOLLOWING TIMES ARE USED:

Intake ends after (yr): $\quad 1.0$

Dose calculations ends after $(y r): \quad 50.0$

$=========$ FILENAMES AND TITLES OF FILES/LIBRARIES USED

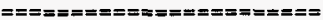

Input file name: IGENII \CWCmfpb. in

GENII Default Parameter Values (28-Mar-90 RAP)

Radionuclide Library - Times $<100$ years (23-JuTy-93 PDR)

External Dose Factors for GENII in person $\mathrm{Sv} / \mathrm{yr}$ per Bq/n (8-May-90 $\mathrm{R}$

PNL Solubilities, Yearly Dose Increments (23-Jul-93 PDR)

\begin{tabular}{|c|c|c|c|}
\hline $\begin{array}{l}\text { Release } \\
\text { Radio- } \\
\text { nuclide }\end{array}$ & $\underset{\mathrm{Ci} / \mathrm{yr}}{\operatorname{Air}}$ & $\begin{array}{l}\text { Surface } \\
\text { Water } \\
\text { Ci/yr }\end{array}$ & $\begin{array}{r}\text { Buried } \\
\text { Source } \\
\mathrm{Ci} / \mathrm{mb}^{3}\end{array}$ \\
\hline $\begin{array}{l}\text { CS137 } \\
\text { SR90 } \\
Y 90 \\
\text { PU239 } \\
\text { PU241 } \\
\text { AM241 }\end{array}$ & $\begin{array}{l}2.5 \mathrm{E}-01 \\
1.9 \mathrm{E}-01 \\
1.9 \mathrm{E}-01 \\
4.1 \mathrm{E}-03 \\
1.0 \mathrm{E}-01 \\
7.9 \mathrm{E}-03\end{array}$ & $\begin{array}{l}0.0 \mathrm{E}+00 \\
0.0 \mathrm{E}+00 \\
0.0 \mathrm{E}+00 \\
0.0 \mathrm{E}+00 \\
0.0 \mathrm{E}+00 \\
0.0 \mathrm{E}+00\end{array}$ & $\begin{array}{l}0.0 E+00 \\
0.0 E+00 \\
0.0 E+00 \\
0.0 E+00 \\
0.0 E+00 \\
0.0 E+00\end{array}$ \\
\hline
\end{tabular}


WHC-SD-WM-CN-016, Rev. 0

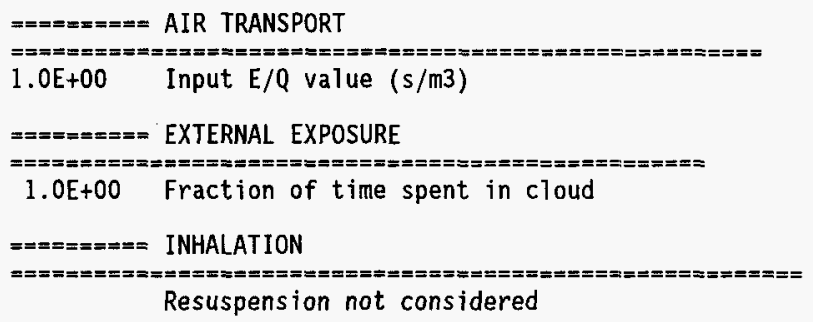

Input prepared by:

Date:

Input checked by:

Date:

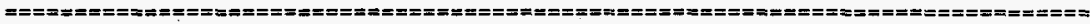

$=$ 
WHC-SD-WM-CN-016, Rev. 0

\section{GENII Dose Calculation Program \\ (Version 1.485 3-Dec-90)}

Case title: CWC - MFP-percent by activity-insoluble Pu. 1

Executed on: $02 / 28 / 96$ at $09: 58: 24$

Page $C$.

Acute release

Uptake/exposure period:

Dose commitment period:

Dose units:

1.0

50.0

Rem

Committed Weighting Whated
Dose

\begin{tabular}{lccc} 
Organ & Equivalent & Factors & Equivalent \\
\hline Gonads & $3.9 \mathrm{E}+02$ & $2.5 \mathrm{E}-01$ & $9.7 \mathrm{E}+01$ \\
Breast & $2.6 \mathrm{E}+00$ & $1.5 \mathrm{E}-01$ & $3.9 \mathrm{E}-01$ \\
R Marrow & $2.2 \mathrm{E}+03$ & $1.2 \mathrm{E}-01$ & $2.7 \mathrm{E}+02$ \\
Lung & $2.2 \mathrm{E}+03$ & $1.2 \mathrm{E}-01$ & $2.6 \mathrm{E}+02$ \\
Thyroid & $2.6 \mathrm{E}+00$ & $3.0 \mathrm{E}-02$ & $7.7 \mathrm{E}-02$ \\
Bone Sur & $2.8 \mathrm{E}+04$ & $3.0 \mathrm{E}-02$ & $8.3 \mathrm{E}+02$ \\
Liver & $4.9 \mathrm{E}+03$ & $6.0 \mathrm{E}-02$ & $2.9 \mathrm{E}+02$ \\
LL Int. & $7.0 \mathrm{E}+00$ & $6.0 \mathrm{E}-02$ & $4.2 \mathrm{E}-01$ \\
UL Int. & $4.4 \mathrm{E}+00$ & $6.0 \mathrm{E}-02$ & $2.6 \mathrm{E}-01$ \\
S Int. & $3.2 \mathrm{E}+00$ & $6.0 \mathrm{E}-02$ & $1.9 \mathrm{E}-01$ \\
Stomach & $2.9 \mathrm{E}+00$ & $6.0 \mathrm{E}-02$ & $1.7 \mathrm{E}-01$ \\
\hline Internal Effective Dose Equivalent & $1.8 \mathrm{E}+03$ \\
External Dose & \multicolumn{3}{c}{}
\end{tabular}

Annual Effective Dose Equivalent $\quad 1.8 \mathrm{E}+03$

Controlling Organ:

Controlling Pathway:

Controlling Radionuclide:

Total Inhalation EDE:

Total Ingestion EDE:
Bone Sur

Inh

AM241

$1.8 \mathrm{E}+03$

$0.0 \mathrm{E}+00$ 
WHC-SD-WM-CN-016, Rev. 0 
WHC-SD-WM-CN-016, Rev. 0

\section{GENII Dose Calculation Program}

(Version 1.485 3-Dec-90)

Case title: CWC - MFP-percent by activity-insoluble Pu.

Executed on: $02 / 28 / 96$ at $09: 58: 24$

Page $C$. 2

Acute release

Uptake/exposure period:

1.0

Dose commitment period:

Dose units:

50.0

Rem

Dose Commitment Year

$\begin{array}{llll}1 & 2 & 3 & \ldots\end{array}$

Internal : |

Intake : : I

Year: 3

$0.0 E+00 \quad \ldots$

21

$0.0 \mathrm{E}+00$

$0.0 E+00$

Internal

I

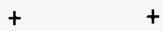

Effective

$1: 9.9 E+01+6.7 E+01+6.0 E+01+\ldots=1.8 E+03$ Dose

Equivalent

i! I1 H

Interna1

Cumulative

Annua 1

$9.9 \mathrm{E}+01+6.7 \mathrm{E}+01+6.0 \mathrm{E}+01+\ldots=1.8 \mathrm{E}+03$

Internal

Dose

Dose

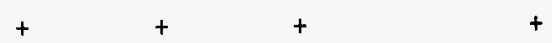

External 
WHC-SD-WM-CN-016, Rev. 0

$\begin{array}{llllll}\text { Annual } & 2.9 \mathrm{E}-02 & 0.0 \mathrm{E}+00 & 0.0 \mathrm{E}+00 & \ldots & 2.9 \mathrm{E}-02\end{array}$

Dose

I II II II

Annua 1

Dose

$9.9 \mathrm{E}+01+6.7 \mathrm{E}+01+6.0 \mathrm{E}+01+\ldots=1.8 \mathrm{E}+03$ Dose

Maximum

9.9E+01 Annual

Dose Occurred

In Year 1 
WHC-SD-WM-CN-016, Rev. 0

GENI I Dose Calculation Program

(Version 1.485 3-Dec-90)

Case title: CWC - MFP-percent by activity-insoluble Pu.

Executed on: $02 / 28 / 96$ at $09: 58: 24$

Page $C$. 3

Acute release

Uptake/exposure period:

Dose commitment period:

Dose units:

$$
\text { Rem } \begin{array}{r}
1.0 \\
50.0
\end{array}
$$

Committed Dose Equivalent by Exposure Pathway

Pathway Lung Stomach S Int. UL. Int. LL Int. Bone Su R Marro Testes

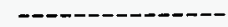

Inhale

$3.9 \mathrm{E}+02$

Total

3. $9 E+02$

\begin{tabular}{lllll} 
Pathway & Ovaries Muscle & Thyroid Liver \\
\hline Inhale & $3.8 \mathrm{E}+02$ & $2.6 \mathrm{E}+00$ & $2.6 \mathrm{E}+00$ & $4.9 \mathrm{E}+03$ \\
\hdashline Total & $3.8 \mathrm{E}+02$ & $2.6 \mathrm{E}+00$ & $2.6 \mathrm{E}+00$ & $4.9 \mathrm{E}+03$
\end{tabular}

\begin{tabular}{ll} 
Pathway & \\
\hline Plume & $--9 \mathrm{E}-02$ \\
\hline Total & $\mathbf{2 . 9 \mathrm { E } - 0 2}$
\end{tabular}




\section{GENII Dose Calculation Program}

(Version 1.485 3-Dec-90)

Case title: CWC - MFP-percent by activity-insoluble Pu.

4

Executed on: $02 / 28 / 96$ at $09: 58: 24$ Page $C$.

Acute release

Uptake/exposure period:

Dose commitment period:

Dose units:

$$
\text { Rem } \begin{array}{r}
1.0 \\
50.0
\end{array}
$$

Committed Dose Equivalent by Radionuclide

Radionuclide Lung Stomach S Int. UL Int. LL Int. Bone Su R Marro Testes

SR 90

8.9E-02

$Y 90$

$1.3 \mathrm{E}-04$

CS 137

2.7E+00

PU 241

3. $2 \mathrm{E}+01$

U 237

$0.0 E+00$

AM 241

$3.0 \mathrm{E}+02$

PU 239

$5.9 E+01$

Total

3. $9 \mathrm{E}+02$ $\begin{array}{llllll}3.5 \mathrm{E}-01 & 9.6 \mathrm{E}-02 & 1.0 \mathrm{E}-01 & 2.3 \mathrm{E}-01 & 7.2 \mathrm{E}-01 \quad 1.5 \mathrm{E}+02 \quad 6.8 \mathrm{E}+01\end{array}$

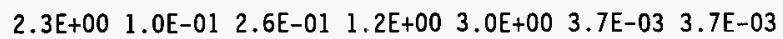

$2.7 \mathrm{E}+00 \quad 2.7 \mathrm{E}+00 \quad 2.8 \mathrm{E}+00 \quad 2.8 \mathrm{E}+00 \quad 2.8 \mathrm{E}+00 \quad 2.5 \mathrm{E}+00 \quad 2.6 \mathrm{E}+00$

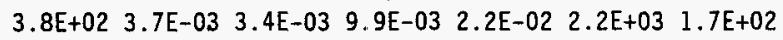

$\begin{array}{llllllll}0.0 E+00 & 0.0 E+00 & 0.0 E+00 & 0.0 E+00 & 0.0 E+00 & 0.0 E+00 & 0.0 E+00\end{array}$

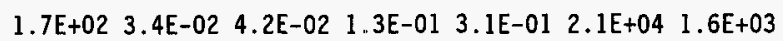

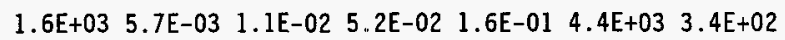

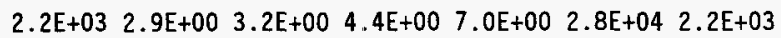

\begin{tabular}{lllll} 
Radionuclide & Ovaries Muscle & Thyroid Liver \\
\hline SR 90 & $8.9 \mathrm{E}-02$ & $8.6 \mathrm{E}-02$ & $8.9 \mathrm{E}-02$ & $0.0 \mathrm{E}+00$ \\
Y 90 & $1.2 \mathrm{E}-04$ & $1.2 \mathrm{E}-04$ & $1.2 \mathrm{E}-04$ & $3.7 \mathrm{E}-03$ \\
CS 137 & $2.5 \mathrm{E}+00$ & $2.4 \mathrm{E}+00$ & $2.4 \mathrm{E}+00$ & $0.0 \mathrm{E}+00$ \\
PU 241 & $3.1 \mathrm{E}+01$ & $3.4 \mathrm{E}-03$ & $1.5 \mathrm{E}-03$ & $3.7 \mathrm{E}+02$ \\
U 237 & $0.0 \mathrm{E}+00$ & $0.0 \mathrm{E}+00$ & $0.0 \mathrm{E}+00$ & $0.0 \mathrm{E}+00$ \\
AM 241 & $2.9 \mathrm{E}+02$ & $2.9 \mathrm{E}-02$ & $1.6 \mathrm{E}-02$ & $3.8 \mathrm{E}+03$
\end{tabular}


WHC-SD-WM-CN-016, Rev, 0

PU 239

$5.9 \mathrm{E}+01 \quad 2.1 \mathrm{E}-03 \quad 2.0 \mathrm{E}-03 \quad 7.8 \mathrm{E}+02$

Total

$3.8 \mathrm{E}+02 \quad 2.6 \mathrm{E}+00 \quad 2.6 \mathrm{E}+00 \quad 4.9 \mathrm{E}+03$

Total 
WHC-SD-WM-CN-016, Rev. 0

GENII Dose CaTculation Program

(Version 1.485 3-Dec-90)

Case title: CWC - MFP-percent by activity-insoluble Pu.

Executed on: $02 / 28 / 96$ at $09: 58: 24$

Page C. 5

Acute release

Uptake/exposure period: Dose commitment period:

Dose units:

Inhalation Ingestion

Effective Effective

Radionuclide

SR 90

Y 90

CS 137

PU 241

U 237

AM 241

PU 239
Dose

Equivalent Equivalent

$$
\text { 1. } 3 \mathrm{E}+01
$$

5. $5 \mathrm{E}-01$

2. $5 \mathrm{E}+00$

1. $6 \mathrm{E}+02$

$0.0 \mathrm{E}+00$

1. $2 \mathrm{E}+03$

4. $3 \mathrm{E}+02$
- 0 .

$0.0 \mathrm{E}+00$

$0.0 \mathrm{E}+00$

$0.0 \mathrm{E}+00$

$0.0 \mathrm{E}+00$

$0.0 E+00$

$0.0 \mathrm{E}+00$

$0.0 \mathrm{E}+00$
External

Dose

$7.8 \mathrm{E}-06$

2.8E-04

2.9E-02

7.7E-12

$0.0 \mathrm{E}+00$

$1.7 \mathrm{E}-05$

8. $8 \mathrm{E}-08$
1.0

50.0

Rem

Internal Annual

Effective Effective Dose Dose

Equivalent Equivalent

$1.3 \mathrm{E}+01 \quad 1.3 \mathrm{E}+01$

$1.3 E+01$

5. 5E-01 5.5E-01

2. $5 \mathrm{E}+00 \quad 2.5 \mathrm{E}+00$

1. $6 \mathrm{E}+02 \quad 1.6 \mathrm{E}+02$

$0.0 E+00 \quad 0.0 E+00$

1. $2 \mathrm{E}+03 \quad 1.2 \mathrm{E}+03$

4. $3 \mathrm{E}+02 \quad 4.3 \mathrm{E}+02$ 
\#\#\#\#\#\#\#\#\#\#\#\#\#\#\#\#\#\#\#\# Program GENII Input File \#\#\#\#\#\#\#\#\#\# 8 Jul 88 \#\#\#\#

Title: CWC - 12 percent nominal Pu-percent by weight-soluble $\mathrm{Pu}$.

$\backslash$ GENII $\backslash$ CWCPU: in

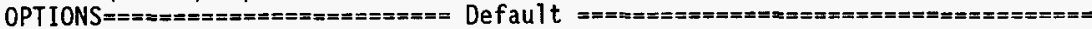

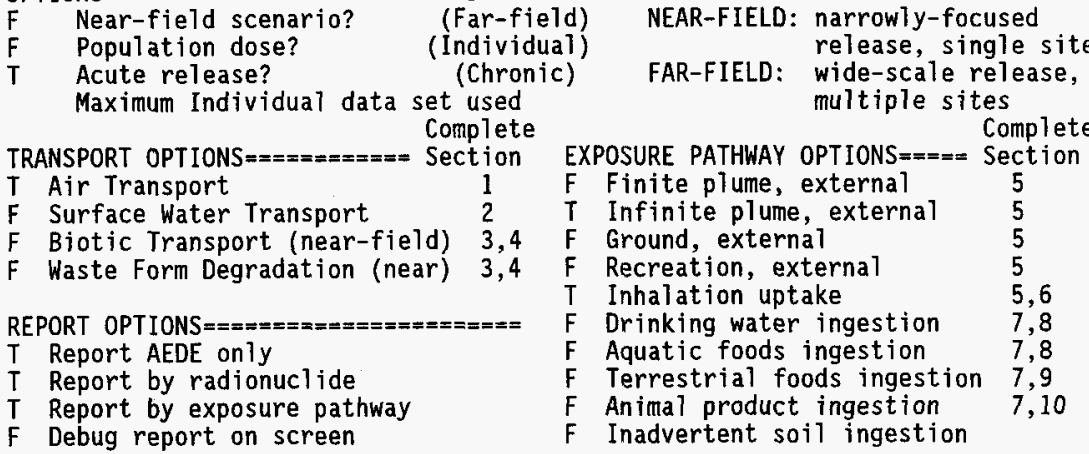

\section{INVENTORY \#\#\#\#\#\#\#\#\#\#\#\#\#\#\#\#\#\#\#\#\#\#\#\#\#\#\#\#\#\#\#\#\#\#\#\#\#\#\#\#\#\#\#\#\#\#\#\#\#\#\#\#\#\#\#\#\#\#\#\#\#\#\#\#\#\#}

4 Inventory input activity units: (1-pCi 2-uCi 3-mCi 4-Ci 5-Bq)

0 Surface soil source units $\left(1-\mathrm{m}_{2} 2-\mathrm{m} 3 \quad 3-\mathrm{kg}\right)$

Equilibrium question goes here

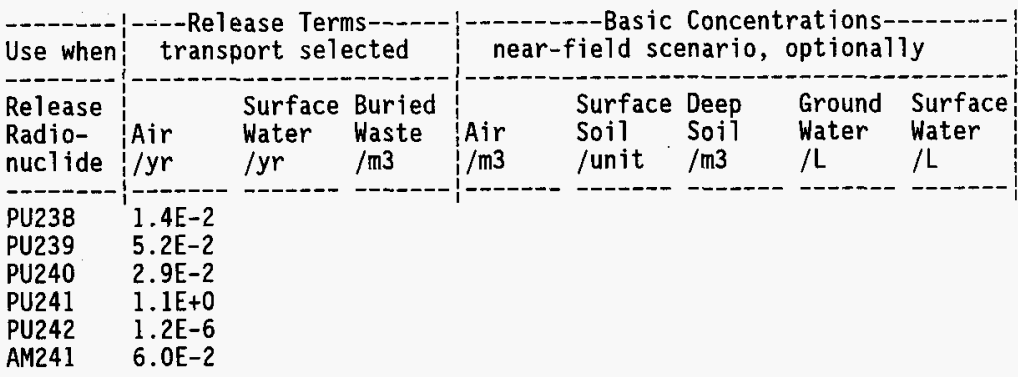

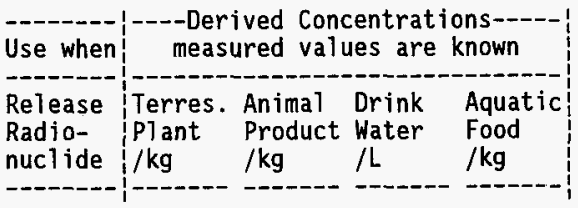

TIME \#\#\#\#\#\#\#\#\#\#\#\#\#\#\#\#\#\#\#\#\#\#\#\#\#\#\#\#\#\#\#\#\#\#\#\#\#\#\#\#\#\#\#\#\#\#\#\#\#\#\#\#\#\#\#\#\#\#\#\#\#\#\#\#\#\#\#\#\#\#

$1 \quad$ Intake ends after (yr)

50 Dose calc. ends after (yr)

0 Release ends after (yr) 
WHC-SD-WM-CN-016, Rev. 0

0 No. of years of air deposition prior to the intake period

0 No. of years of irrigation water deposition prior to the intake period

FAR-FIELD SCENARIOS (IF POPULATION DOSE) \#\#\#\#\#\#\#\#\#\#\#\#\#\#\#\#\#\#\#\#\#\#\#\#\#\#\#\#\#\#\#\#\#

0

0

NEAR-FIELD SCENARIOS \#\#\#\#\#\#\#\#\#\#\#\#\#\#\#\#\#\#\#\#\#\#\#\#\#\#\#\#\#\#\#\#\#\#\#\#\#\#\#\#\#\#\#\#\#\#\#\#\#\#\#\#\#\#\#\#

Prior to the beginning of the intake period: (yr)

When was the inventory disposed? (Package degradation starts)

When was LOIC? (Biotic transport starts)

Fraction of roots in upper soil (top $15 \mathrm{~cm}$ )

Fraction of roots in deep soil

Manual redistribution: deep soil/surface soil dilution factor

0 Source area for external dose modification factor (m2)

TRANSPORT \#\#\#\#\#\#\#\#\#\#\#\#\#\#\#\#\#\#\#\#\#\#\#\#\#\#\#\#\#\#\#\#\#\#\#\#\#\#\#\#\#\#\#\#\#\#\#\#\#\#\#\#\#\#\#\#\#\#\#\#\#\#\#\#\#\#\#\#

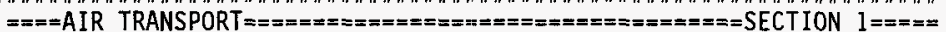

1

0 -Calculate PM

Option: 1-Use chi/Q or PM value IF

2-Select MI dist \& dir 0

3-Specify MI dist \& dir 0

Chi/Q or PM value

MI sector index $(1=S)$

10

1

0

$\mathrm{T}$

MI distance from release point (m) 0

Use jf data, (T/F) else chi/Q grid 0
Release type (0-3)

Stack release $(T / F)$

Stack height (m)

Stack flow $(\mathrm{m} 3 / \mathrm{sec})$

Stack radius $(\mathrm{m})$

Effluent temp. (C)

Building $x$-section (m2)

Building height $(\mathrm{m})$

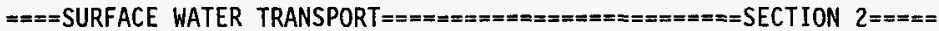
Mixing ratio model: 0-use value, 1-river, 2-lake

Mixing ratio, dimensionless

Average river flow rate for: MIXFLG=0 (m3/s), MIXFLG=1,2 (m/s), Transit time to irrigation withdrawl location (hr)

If mixing ratio model $>0$ :

Rate of effluent discharge to receiving water body (m3/s)

Longshore distance from release point to usage location (m)

offshore distance to the water intake (m)

Average water depth in surface water body $(\mathrm{m})$

Average river width (m), MIXFLG $=1$ only

Depth of effluent discharge point to surface water $(m)$, lake only

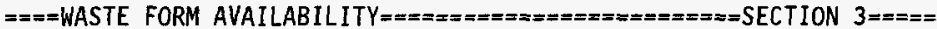
Waste form/package half life, (yr)

Waste thickness, (m)

Depth of soil overburden, $m$

$====$ BIOTIC TRANSPORT OF BURIED SOURCE $== \pm==========x=$ SECTION $4=====$

T Consider during inventory decay/buildup period (T/F)?

0

Pre-Intake site condition............ 2-Humid non agricultural 3-Agriculturai

EXPOSURE \#\#\#\#\#\#\#\#\#\#\#\#\#\#\#\#\#\#\#\#\#\#\#\#\#\#\#\#\#\#\#\#\#\#\#\#\#\#\#\#\#\#\#\#\#\#\#\#\#\#\#\#\#\#\#\#\#\#\#\#\#\#\#\#\#\#\#\#\# 
WHC-SD-WM-CN-016, Rev. 0

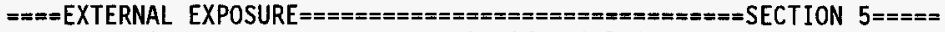
Exposure time:

Plume (hr)

Soil contamination (hr)

Swimming (hr)

Boating (hr)

Residential irrigation:

$\mathrm{T}$

Consider: (T/F)

Shoreline activities (hr)

3-ocean, 4-tidal basin)

Transit

Average fraction of time submersed in acute cloud (hr/person $\mathrm{hr}$ )

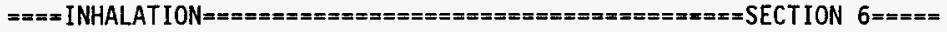
Hours of exposure to contamination per year

0 -No resus- 1-Use Mass Loading pension Mass loading factor $(\mathrm{g} / \mathrm{m} 3)$. Top soil available $(\mathrm{cm})$

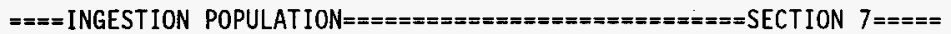
Atmospheric production definition (select option):

0 -Use food-weighted chi $/ Q,($ food-sec $/ \mathrm{m} 3)$, enter value on this 1 ine 1-Use population-weighted chi/Q

2-Use uniform production

3-Use chi/Q and production grids (PRODUCTION will be overridden) Population ingesting aquatic foods, 0 defaults to total (person) Population ingesting drinking water, 0 defaults to total (person) Consider dose from food exported out of region (default $=F$ )

Note below: $S^{*}$ or Source: 0-none, 1-ground water, 2-surface water 3-Derived concentration entered above AQUATIC FOODS / DRINKING WATER INGESTION $=========$ SECTION $8====$

Salt water? (default is fresh)

\begin{tabular}{|c|c|c|c|c|c|c|}
\hline $\begin{array}{l}\text { USE } \\
? \\
\text { T/F }\end{array}$ & $\begin{array}{l}\text { FOOD } \\
\text { TYPE }\end{array}$ & $\begin{array}{l}\text { TRAN- } \\
\text { SIT } \\
\mathrm{hr}\end{array}$ & $\begin{array}{l}\text { PROD- } \\
\text { UCTION } \\
\mathrm{kg} / \mathrm{yr}\end{array}$ & $\begin{array}{l}\text {-CONSUMF } \\
\text { HOLDUP } \\
\mathrm{da}\end{array}$ & $\begin{array}{l}\text { PTION- } \\
\text { RATE } \\
\mathrm{kg} / \mathrm{yr}\end{array}$ & DRINKING WATER \\
\hline $\begin{array}{l}F \\
F \\
F\end{array}$ & $\begin{array}{l}\text { FISH } \\
\text { MOLLUS } \\
\text { CRUSTA } \\
\text { PLANTS }\end{array}$ & $\begin{array}{l}0.00 \\
0.00 \\
0.00 \\
0.00\end{array}$ & $\begin{array}{l}0.0 \\
0.0 \\
0.0 \\
0.0\end{array}$ & $\begin{array}{l}0.00 \\
0.00 \\
0.00 \\
0.00\end{array}$ & $\begin{array}{l}0.0 \\
0.0 \\
0.0 \\
0.0\end{array}$ & $\begin{array}{l}\text { Source (see above) } \\
\text { Treatment? T/F } \\
\text { H]dup/transit (da) } \\
\text { Consumpton (L/yr) }\end{array}$ \\
\hline
\end{tabular}

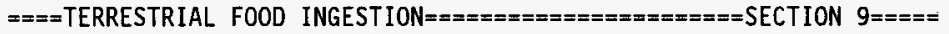

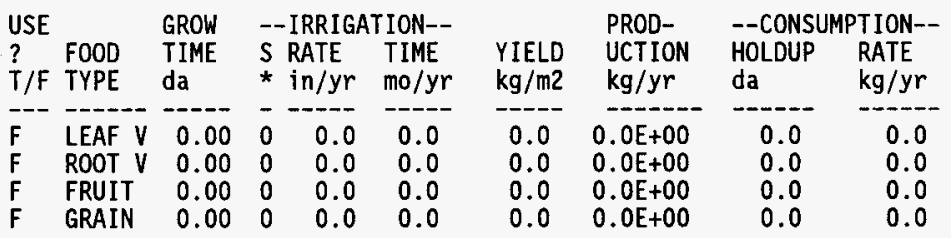


WHC-SD-WM-CN-016, Rev. 0

\begin{tabular}{|c|c|c|c|c|c|c|c|c|c|c|c|}
\hline \multirow[b]{2}{*}{$\begin{array}{l}\text { USE } \\
\stackrel{?}{\mathrm{~T} / \mathrm{F}}\end{array}$} & \multirow[b]{2}{*}{$\begin{array}{l}\text { FOOD. } \\
\text { TYPE }\end{array}$} & & \multirow{2}{*}{$\begin{array}{l}\text { TOTAL } \\
\text { PROD- } \\
\text { UCTION } \\
\mathrm{kg} / \mathrm{yr}\end{array}$} & \multirow{2}{*}{$\begin{array}{l}\text { DRINK } \\
\text { WATER } \\
\text { CONTAM } \\
\text { FRACT. }\end{array}$} & \multicolumn{6}{|c|}{ - STPPFD } \\
\hline & & $\begin{array}{l}\text { CONSUM } \\
\text { RATE } \\
\mathrm{kg} / \mathrm{yr}\end{array}$ & $\begin{array}{l}\text { PTION } \\
\text { HOLDUP } \\
\text { da }\end{array}$ & & & $\begin{array}{l}\text { DIET } \\
\text { FRAC- } \\
\text { TION }\end{array}$ & $\begin{array}{c}\text { GROW } \\
\text { TIME } \\
\mathrm{da}\end{array}$ & $\begin{array}{l}\text { - IRRIGA } \\
\text { S RATE } \\
\text { * in } / y r\end{array}$ & $\begin{array}{l}\text { TION-- } \\
\text { TIME } \\
\text { mo/yr }\end{array}$ & $\begin{array}{l}\text { YIELD } \\
\mathrm{kg} / \mathrm{m} 3\end{array}$ & $\begin{array}{l}\text { STOR- } \\
\text { AGE } \\
\text { da }\end{array}$ \\
\hline$F$ & $\begin{array}{l}\text { BEEF } \\
\text { POULTR } \\
\text { MILK } \\
\text { EGG }\end{array}$ & $\begin{array}{l}0.0 \\
0.0 \\
0.0 \\
0.0\end{array}$ & $\begin{array}{l}0.0 \\
0.0 \\
0.0 \\
0.0\end{array}$ & $\begin{array}{l}0.00 \\
0.00 \\
0.00 \\
0.00\end{array}$ & $\begin{array}{l}0.00 \\
0.00 \\
0.00 \\
0.00\end{array}$ & $\begin{array}{l}0.00 \\
0.00 \\
0.00 \\
0.00\end{array}$ & $\begin{array}{l}0.0 \\
0.0 \\
0.0 \\
0.0\end{array}$ & $\begin{array}{l}0.0 \\
0.0 \\
0.0 \\
0.0\end{array}$ & $\begin{array}{l}0.00 \\
0.00 \\
0.00 \\
0.00\end{array}$ & $\begin{array}{l}0.00 \\
0.00 \\
0.00 \\
0.00\end{array}$ & $\begin{array}{l}0.0 \\
0.0 \\
0.0 \\
0.0\end{array}$ \\
\hline & $\begin{array}{l}\text { BEEF } \\
\text { MILK }\end{array}$ & & & & & $\begin{array}{l}0.00 \\
0.00\end{array}$ & $\begin{array}{l}0.0 \\
0.0\end{array}$ & $\begin{array}{l}0.0 \\
0.0\end{array}$ & $\begin{array}{l}0.00 \\
0.00\end{array}$ & $\begin{array}{l}0.00 \\
0.00\end{array}$ & $\begin{array}{l}0.0 \\
0.0\end{array}$ \\
\hline
\end{tabular}

\#\#\#\#\#\#\#\#\#\#\#\#\#\#\#\#\#\#\#\#\#\#\#\#\#\#\#\#\#\#\#\#\#\#\#\#\#\#\#\#\#\#\#\#\#\#\#\#\#\#\#\#\#\#\#\#\#\#\#\#\#\#\#\#\#\#\#\#\#\#\#\#\#\#\# 


\section{GENII Dose Calculation Program}

(Version 1.485 3-Dec-90)

Case title: CWC - 12 percent nominal Pu-percent by weight-soluble Pu. 1

Executed on: $02 / 28 / 96$ at $10: 14: 12$

Page $A$.

This is a far-field (wide-scale release, multiple site) scenario.

Release is acute

Individual dose

THE FOLLOWING TRANSPORT MODES ARE CONSIDERED

Air

THE FOLLOWING EXPOSURE PATHS ARE. CONSIDERED:

Infinite plume, external

Inhalation uptake

THE FOLLOWING TIMES ARE USED:

Intake ends after $(y r):$

Dose calculations ends after (yr): 50.0

$=========$ FILENAMES AND TITLES OF FILES/LIBRARIES. USED

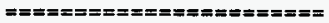

Input file name: IGENII \CWCPu.in

GENII Default Parameter Values (28-Mar-90 RAP)

Radionuclide Library - Times $<100$ years (23-JuTy-93 PDR)

External Dose Factors for GENII in person Sv/yr per Bq/n (8-May-90 R

Worst-Case Solubilities, Yearly Dose Increments (23-Jul-93 PDR)

\begin{tabular}{|c|c|c|c|}
\hline Release & & Surface & Buried \\
\hline $\begin{array}{l}\text { Radio- } \\
\text { nuclide }\end{array}$ & $\underset{\mathrm{Ci} / \mathrm{yr}}{\operatorname{Air}}$ & $\begin{array}{l}\text { Water } \\
\text { Ci/yr }\end{array}$ & $\begin{array}{r}\text { Source } \\
\mathrm{C} i / \mathrm{m} 3\end{array}$ \\
\hline $\begin{array}{l}\text { PU239 } \\
\text { PU240 } \\
\text { PU241 }\end{array}$ & & & $\begin{array}{l}0.0 \mathrm{E}+00 \\
0.0 \mathrm{E}+00 \\
0.0 \mathrm{E}+00 \\
0.0 \mathrm{E}+00\end{array}$ \\
\hline
\end{tabular}


WHC-SD-WM-CN-016, Rev. 0

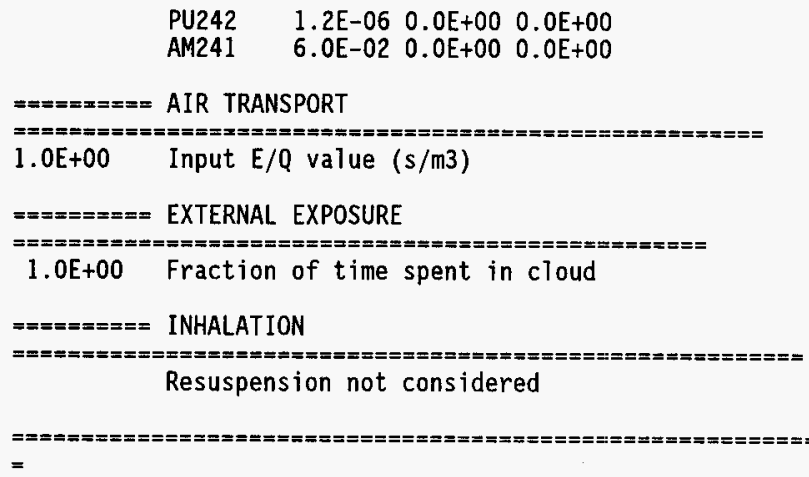


Case title: CWC - 12 percent nominal Pu-percent by weight-soluble Pu.

Executed on: $02 / 28 / 96$ at $10: 14: 29$

Page C. 1

Acute release

Uptake/exposure period:

Dose commitment period:

Dose units:

$$
\text { Rem } \begin{array}{r}
1.0 \\
50.0
\end{array}
$$

\begin{tabular}{|c|c|c|c|}
\hline Organ & $\begin{array}{l}\text { Committed } \\
\text { Dose } \\
\text { Equivalent }\end{array}$ & $\begin{array}{l}\text { Weighting } \\
\text { Factors }\end{array}$ & $\begin{array}{l}\text { Weighted } \\
\text { Dose } \\
\text { Equivalent }\end{array}$ \\
\hline $\begin{array}{l}\text { Gonads } \\
\text { Breast } \\
\text { R Marrow } \\
\text { Lung } \\
\text { Thyroid } \\
\text { Bone Sur } \\
\text { Liver } \\
\text { LL Int. } \\
\text { UL Int. } \\
\text { S Int. } \\
\text { Stomach }\end{array}$ & $\begin{array}{l}6.5 \mathrm{E}+03 \\
3.8 \mathrm{E}-01 \\
3.6 \mathrm{E}+04 \\
3.3 \mathrm{E}+03 \\
2.5 \mathrm{E}-01 \\
4.6 \mathrm{E}+05 \\
8.1 \mathrm{E}+04 \\
5.8 \mathrm{E}+00 \\
2.2 \mathrm{E}+00 \\
6.6 \mathrm{E}-01 \\
4.8 \mathrm{E}-01\end{array}$ & $\begin{array}{l}2 . \\
1 . \\
1 . \\
1 . \\
3 . \\
3 . \\
6 . \\
6 . \\
6 . \\
6 .\end{array}$ & $\begin{array}{l}1.6 \mathrm{E} \\
5.8 \mathrm{E} \\
4.3 \mathrm{E} \\
4.0 \mathrm{E} \\
7.6 \mathrm{E} \\
1.4 \mathrm{E} \\
4.8 \mathrm{E} \\
3.5 \mathrm{E} \\
1.3 \mathrm{E} \\
4.0 \mathrm{E} \\
2.9 \mathrm{E}\end{array}$ \\
\hline \multicolumn{3}{|c|}{$\begin{array}{l}\text { Internal Effective Dose Equivalent } \\
\text { External Dose }\end{array}$} & $\begin{array}{l}2.5 \\
1.3\end{array}$ \\
\hline
\end{tabular}

$\begin{array}{ll}\text { Controlling Organ: } & \text { Bone Sur } \\ \text { Controlling Pathway: } & \text { Inh } \\ \text { Controlling Radionuclide: } & \text { AM241 }\end{array}$


WHC-SD-WM-CN-016, Rev. 0

Total Inhalation EDE:

2. $5 E+04$

Total Ingestion EDE:

$0.0 \mathrm{E}+00$ 


\section{GENI I Dose CaTcuTation Program \\ (Version $1.4853-$ Dec-90)}

Case title: CWC - 12 percent nominal Pu-percent by weight-soluble Pu.

Executed on: $02 / 28 / 96$ at $10: 14: 29$

2

Acute release

Uptake/exposure period:

Dose commitment period:

Dose units:

Page $C$.

Dose Commitment Year

$\begin{array}{llll}1 & 2 & 3 & \ldots\end{array}$

Internal : 1

Intake : I

Year: 3 i

$0.0 \mathrm{E}+00 \quad \cdots$

i

$+$

$2: 0.0 \mathrm{E}+00 \quad 0.0 \mathrm{E}+00 \quad \ldots \quad$ Interna

I

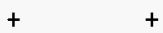

Effective

$1: 9.9 \mathrm{E}+02+6.8 \mathrm{E}+02+6.7 \mathrm{E}+02+\ldots=2.5 \mathrm{E}+04$ Dose

Equivalent

il $11 \quad H$

Internal

1

Annual

$9.9 \mathrm{E}+02+6.8 \mathrm{E}+02+6.7 \mathrm{E}+02+\ldots=2.5 \mathrm{E}+04$

Cumu 1 ative

Dose

Dose

$+\quad+\quad+$

Externa1 
WHC-SD-WM-CN-016, Rev. 0

Annua $] \quad 1.3 \mathrm{E}-04 \quad 0.0 \mathrm{E}+00 \quad 0.0 \mathrm{E}+00 \quad \ldots \quad 1.3 \mathrm{E}-04$

Dose

Annual
i i
I i
i i
i i

Annual

$9.9 \mathrm{E}+02+6.8 \mathrm{E}+02+6.7 \mathrm{E}+02+\ldots=2.5 \mathrm{E}+04 \quad$ Cumulative
Dose

Maximum

9.9E+02 Annual

Dose Occurred

In Year 1 


\section{GENII Dose Calculation Program \\ (Version 1.485 3-Dec-90)}

Case title: CWC - 12 percent nominal Pu-percent by weight-soluble Pu. Executed on: $02 / 28 / 96$ at $10: 14: 29$

Page $C$. 3

Acute release

Uptake/exposure period:

Dose commitment period:

Dose units:

$\operatorname{Rem}^{1.0} \begin{array}{r}50.0 \\ \end{array}$

Committed Dose Equivalent by Exposure Pathway

Pathway Lung Stomach S Int. UL Int. LL Int. Bone Su R Marro Testes

------------.--

Inhale

$6.5 \mathrm{E}+03$

$\begin{array}{lllll}3.3 \mathrm{E}+03 \quad 4.8 \mathrm{E}-01 \quad 6.6 \mathrm{E}-01 & 2.2 \mathrm{E}+00 \quad 5.8 \mathrm{E}+00 \quad 4.6 \mathrm{E}+05 \quad 3.6 \mathrm{E}+04\end{array}$

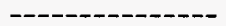

Total

$\begin{array}{lllll}3.3 \mathrm{E}+03 \quad 4.8 \mathrm{E}-01 \quad 6.6 \mathrm{E}-01 \quad 2.2 \mathrm{E}+00 \quad 5.8 \mathrm{E}+00 \quad 4.6 \mathrm{E}+05 \quad 3.6 \mathrm{E}+04 & 4\end{array}$

$6.5 \mathrm{E}+03$

\begin{tabular}{lllll} 
Pathway & Ovaries Muscle & Thyroid Liver \\
\hline Inhale & $6.4 \mathrm{E}+03$ & $3.8 \mathrm{E}-01$ & $2.5 \mathrm{E}-01$ & $8.1 \mathrm{E}+04$ \\
\hline Total & $6.4 \mathrm{E}+03$ & $3.8 \mathrm{E}-01$ & $2.5 \mathrm{E}-01$ & $\mathbf{3 . 1 \mathrm { E } + 0 4}$
\end{tabular}

External Dose by Exposure Pathway

Pathway

\begin{tabular}{ll} 
Plume & $\begin{array}{l}-1.3 \mathrm{E}-04 \\
\text { Total }\end{array}$ \\
\hline $\mathbf{1 . 3 \mathrm { E } - 0 4}$
\end{tabular}


WHC-SD-WM-CN-016, Rev. 0

GENII Dose Calculation Program

(Version 1.485 3-Dec-90)

Case title: CWC - 12 percent nominal Pu-percent by weight-soluble Pu. 4

Executed on: $02 / 28 / 96$ at $10: 14: 29$

Page C.

Acute release

Uptake/exposure period:

Dose commitment period:

Dose units:

$$
\text { Rem } \begin{array}{r}
1.0 \\
50.0
\end{array}
$$

Committed Dose Equivalent by Radionuclide

Radionuclide Lung Stomach S Int. UL Int. LL Int. Bone Su R Marro Testes

-.-.---

PU 240

1. $1 \mathrm{E}+03$

PU 238

4. $5 \mathrm{E}+02$

PU 242

4.1E-02

PU 241

8.5E+02

U 237

$0.0 E+00$

AM 241

2. $3 E+03$

PU 239

$1.9 \mathrm{E}+03$

Total

$6.5 \mathrm{E}+03$

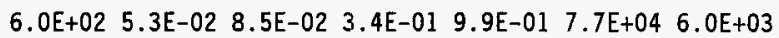

3.1E+02 2.7E-02 4.4E-02 1.9E-01 5.1E-01 3.3E+04 2.6E+03

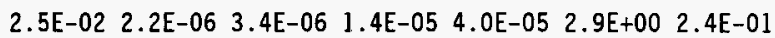

9.9E+00 5.1E-02 5.7E-02 1.3E-01 2.1E-01 5.6E+04 4.4E+03

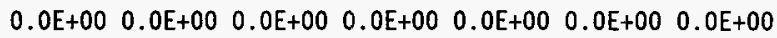

$\begin{array}{lllllll}1.3 \mathrm{E}+03 & 2.6 \mathrm{E}-01 & 3.3 \mathrm{E}-01 & 9.6 \mathrm{E}-01 & 2.4 \mathrm{E}+00 & 1.6 \mathrm{E}+05 & 1.3 \mathrm{E}+04\end{array}$

$\begin{array}{llllll}1.1 \mathrm{E}+03 & 9.4 \mathrm{E}-02 & 1.5 \mathrm{E}-01 & 6.1 \mathrm{E}-01 \quad 1.8 \mathrm{E}+00 & 1.4 \mathrm{E}+05 \quad 1.1 \mathrm{E}+04\end{array}$

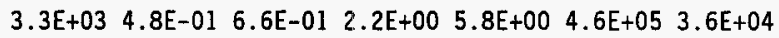

\begin{tabular}{lllll} 
Radionuclide & Ovaries Muscle & Thyroid Liver \\
\hline PU 240 & $1.1 \mathrm{E}+03$ & $3.4 \mathrm{E}-02$ & $3.2 \mathrm{E}-02$ & $1.3 \mathrm{E}+04$ \\
PU 238 & $4.4 \mathrm{E}+02$ & $1.7 \mathrm{E}-02$ & $1.7 \mathrm{E}-02$ & $6.0 \mathrm{E}+03$ \\
PU 242 & $4.2 \mathrm{E}-02$ & $1.5 \mathrm{E}-06$ & $1.3 \mathrm{E}-06$ & $5.3 \mathrm{E}-01$ \\
PU 241 & $8.3 \mathrm{E}+02$ & $5.3 \mathrm{E}-02$ & $2.3 \mathrm{E}-02$ & $8.7 \mathrm{E}+03$ \\
U 237 & $0.0 \mathrm{E}+00$ & $0.0 \mathrm{E}+00$ & $0.0 \mathrm{E}+00$ & $0.0 \mathrm{E}+00$ \\
AM 241 & $2.2 \mathrm{E}+03$ & $2.2 \mathrm{E}-01$ & $1.3 \mathrm{E}-01$ & $2.9 \mathrm{E}+04$ \\
PU 239 & $1.9 \mathrm{E}+03$ & $5.8 \mathrm{E}-02$ & $5.7 \mathrm{E}-02$ & $2.4 \mathrm{E}+04$
\end{tabular}


WHC-SD-WM-CN-016, Rev. 0 
WHC-SD-WM-CN-016, Rev. 0

\section{GENI I Dose Calculation Program}

(Version 1.485 3-Dec-90)

Case title: CWC - 12 percent nominal Pu-percent by weight-soluble Pu.

Executed on: $02 / 28 / 96$ at $10: 14: 29$

Page C.

5

Acute release

Uptake/exposure period:

Dose commitment period:

Dose units:

1.0

50.0

Rem

\begin{tabular}{|c|c|c|c|c|c|}
\hline $\begin{array}{l}\text { Radio- } \\
\text { nuclide }\end{array}$ & $\begin{array}{c}\text { Inhalation } \\
\text { Effective } \\
\text { Dose } \\
\text { Equivalent }\end{array}$ & $\begin{array}{l}\text { Ingestion } \\
\text { Effective } \\
\text { Dose } \\
\text { Equivalent }\end{array}$ & $\begin{array}{c}\text { External } \\
\text { Dose }\end{array}$ & $\begin{array}{l}\text { Internal } \\
\text { Effective } \\
\text { Dose } \\
\text { Equivalent }\end{array}$ & $\begin{array}{c}\text { Annual } \\
\text { Effective } \\
\text { Dose } \\
\text { Equivalent }\end{array}$ \\
\hline $\begin{array}{l}------- \\
\text { PU } 240 \\
\text { PU } 238 \\
\text { PU } 242 \\
\text { PU } 241 \\
\text { U } 237 \\
\text { AM } 241 \\
\text { PU } \\
239\end{array}$ & $\begin{array}{l}4.2 E+03 \\
1.8 E+03 \\
1.6 E-01 \\
2.9 E+03 \\
0.0 E+00 \\
8.9 E+03 \\
7.4 E+03\end{array}$ & $\begin{array}{l}0.0 \mathrm{E}+00 \\
0.0 \mathrm{E}+00 \\
0.0 \mathrm{E}+00 \\
0.0 \mathrm{E}+00 \\
0.0 \mathrm{E}+00 \\
0.0 \mathrm{E}+00 \\
0.0 \mathrm{E}+00\end{array}$ & $\begin{array}{l}8.9 \mathrm{E}-07 \\
4.4 \mathrm{E}-07 \\
0.0 \mathrm{E}+00 \\
8.4 \mathrm{E}-11 \\
0.0 \mathrm{E}+00 \\
1.3 \mathrm{E}-04 \\
1.1 \mathrm{E}-06\end{array}$ & $\begin{array}{l}4.2 \mathrm{E}+03 \\
1.8 \mathrm{E}+03 \\
1.6 \mathrm{E}-01 \\
2.9 \mathrm{E}+03 \\
0.0 \mathrm{E}+00 \\
8.9 \mathrm{E}+03 \\
7.4 \mathrm{E}+03\end{array}$ & $\begin{array}{l}4.2 \mathrm{E}+03 \\
1.8 \mathrm{E}+03 \\
1.6 \mathrm{E}-01 \\
2.9 \mathrm{E}+03 \\
0.0 \mathrm{E}+00 \\
8.9 \mathrm{E}+03 \\
7.4 \mathrm{E}+03\end{array}$ \\
\hline
\end{tabular}


\#\#\#\#\#\#\#\#\#\#\#\#\#\#\#\#\#\#\#\#\# Program GENII Input File \#\#\#\#\#\#\#\#\#\# 8 Jul 88 \#\#\#\# Title: CWC - 12 percent nominal Pu-percent by weight-insoluble Pu. $\backslash$ GENII \CWCpub. in

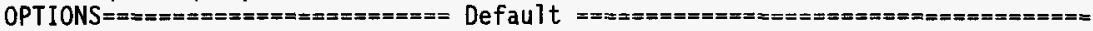

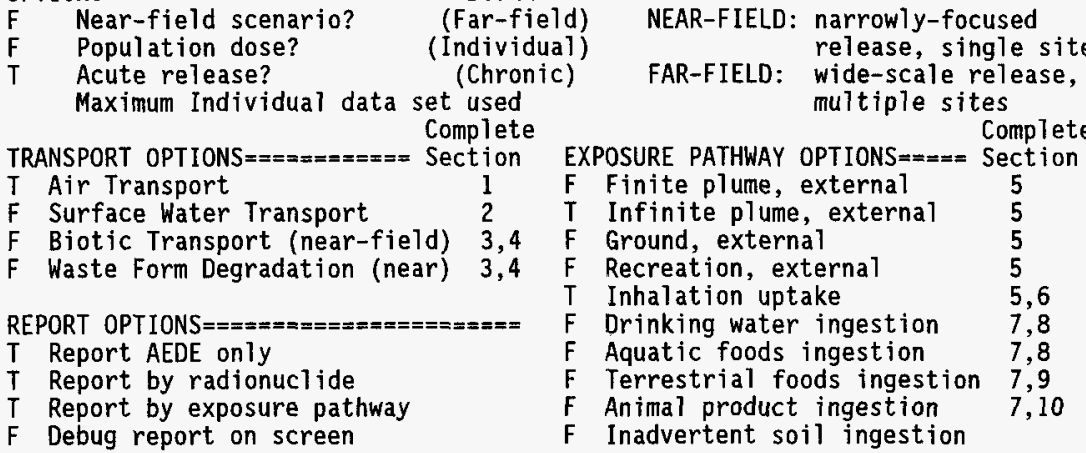

\section{INVENTORY \#\#\#\#\#\#\#\#\#\#\#\#\#\#\#\#\#\#\#\#\#\#\#\#\#\#\#\#\#\#\#\#\#\#\#\#\#\#\#\#\#\#\#\#\#\#\#\#\#\#\#\#\#\#\#\#\#\#\#\#\#\#\#}

4 Inventory input activity units: (1-pCi 2-uCi 3-mCi 4-Ci 5-Bq)

0 Surface soil source units $(1-\mathrm{m} 22-\mathrm{m} 33-\mathrm{kg})$

Equilibrium question goes here

\begin{tabular}{|c|c|c|c|c|c|c|c|c|}
\hline Use wher & & ort sele & ected & & ield sc & enaric & opt 1 & $7 y$ \\
\hline $\begin{array}{l}\text { Release } \\
\text { Radio- } \\
\text { nuclide }\end{array}$ & $\begin{array}{l}\text { Air } \\
/ y r\end{array}$ & $\begin{array}{l}\text { Surface } \\
\text { Water } \\
\text { /yr }\end{array}$ & $\begin{array}{l}\text { Buried } \\
\text { Waste } \\
/ \mathrm{m} 3\end{array}$ & $\begin{array}{l}\text { Air } \\
/ \mathrm{m} 3\end{array}$ & $\begin{array}{l}\text { Surface } \\
\text { Soil } \\
\text { /unit }\end{array}$ & $\begin{array}{l}\text { Deep } \\
\text { Soi1 } \\
/ \mathrm{m} 3\end{array}$ & $\begin{array}{l}\text { Ground } \\
\text { Water } \\
\text { /L }\end{array}$ & $\begin{array}{l}\text { Surface } \\
\text { Water } \\
\text { /L }\end{array}$ \\
\hline
\end{tabular}

PU238 $1.4 \mathrm{E}-2$

PU239 5.2E-2

PU240 2.9E-2

PU241 1.1E+0

PU242 1.2E-6

AM241 $6.0 \mathrm{E}-2$

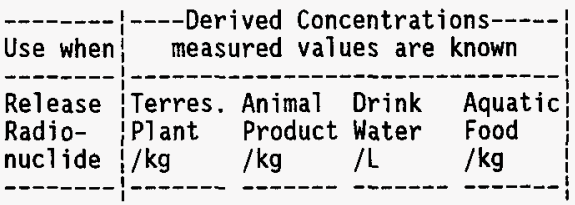

TIME \#\#\#\#\#\#\#\#\#\#\#\#\#\#\#\#\#\#\#\#\#\#\#\#\#\#\#\#\#\#\#\#\#\#\#\#\#\#\#\#\#\#\#\#\#\#\#\#\#\#\#\#\#\#\#\#\#\#\#\#\#\#\#\#\#\#\#\#\#\#\#\#\#

1 Intake ends after (yr)

50 Dose calc. ends after (yr)

0 Release ends after (yr) 
WHC-SD-WM-CN-016, Rev. 0

0 No. of years of air deposition prior to the intake period

0 No. of years of irrigation water deposition prior to the intake period

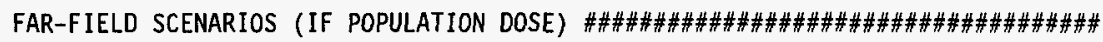

0

0

\section{NEAR-FIELD SCENARIOS \#\#\#\#\#\#\#\#\#\#\#\#\#\#\#\#\#\#\#\#\#\#\#\#\#\#\#\#\#\#\#\#\#\#\#\#\#\#\#\#\#\#\#\#\#\#\#\#\#\#\#}

0

0

0

0

0

0

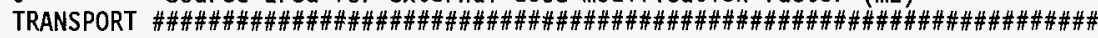

Prior to the beginning of the intake period: (yr)
When was the inventory disposed? (Package degradation starts)

When was LOIC? (Biotic transport starts)

Fraction of roots in upper soil (top $15 \mathrm{~cm}$ )

Fraction of roots in deep soil

Manual redistribution: deep soil/surface soil dilution factor

Source area for external dose modification factor (m2)

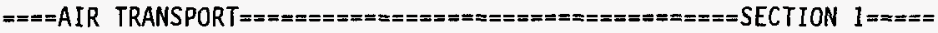

1

0 -Calculate PM

Option: 1-Use chi/Q or PM value 2-Select MI dist \& dir 3-Specify MI dist \& dir

1

0

$\underline{0}$

$\mathrm{T}$

0

0

0

0

0

0

0

0

0

0

0

0

$T$
$T$
0
Chi/Q or PM value

MI sector index $(I=S)$

MI distance from release point (m) 0

Use $j f$ data, (T/F) else chi/Q grid 0
Mixing ratio, dimensionless

If mixing ratio mode $1>0$ : Waste form/package haif life, (yr)

Waste thickness, (m)

Depth of soil overburden, $m$
Release type $(0-3)$

Stack release (T/F)

Stack height (m)

Stack flow (m3/sec)

Stack radius (m)

Effluent temp. (C)

Building $x$-section (m2)

Building height (m)

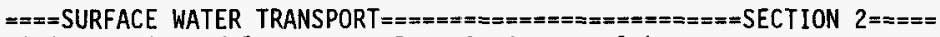
Mixing ratio model: 0-use value, 1-river, 2-lake

Average river flow rate for: MIXFLG $=0(\mathrm{~m} 3 / \mathrm{s}), \operatorname{MIXFLG}=1,2(\mathrm{~m} / \mathrm{s})$, Transit time to irrigation withdrawl location (hr)

Rate of effluent discharge to receiving water body (m3/s)

Longshore distance from release point to usage location (m)

Offshore distance to the water intake $(\mathrm{m})$

Average water depth in surface water body $(\mathrm{m})$

Average river width (m), MIXFLG $=1$ only

Depth of effluent discharge point to surface water (m), lake only

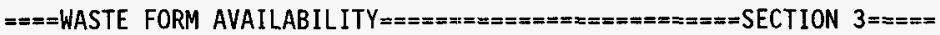

$====$ BIOTIC TRANSPORT OF BURIED SOURCE $====x=m=x=m=====$ SECTION $4=====$ Consider during inventory decay/buildup period (T/F)?

Consider during intake period (T/F)? | l-Arid non agricultural

Pre-Intake site condition............. 2-Humid non agricultural 3-Agriculturai

EXPOSURE \#\#\#\#\#\#\#\#\#\#\#\#\#\#\#\#\#\#\#\#\#\#\#\#\#\#\#\#\#\#\#\#\#\#\#\#\#\#\#\#\#\#\#\#\#\#\#\#\#\#\#\#\#\#\#\#\#\#\#\#\#\#\#\#\#\#\#\# 


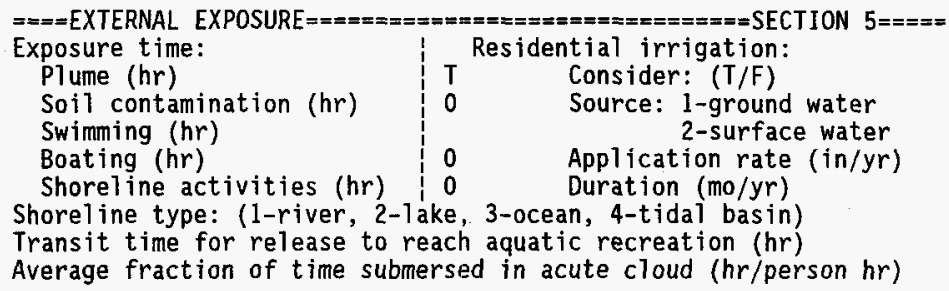

Shoreline type: (1-river, 2-lake, 3-ocean, 4-tidal basin)

Transit time for release to reach aquatic recreation (hr)

Average fraction of time submersed in acute cloud (hr/person hr)

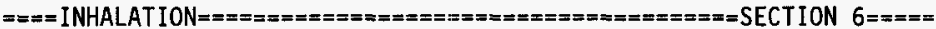
Hours of exposure to contamination per year

0 -No resus- 1 -Use Mass Loading

$$
\text { pension Mass loading factor }(\mathrm{g} / \mathrm{m} 3) \text { Top soil available }(\mathrm{cm})
$$

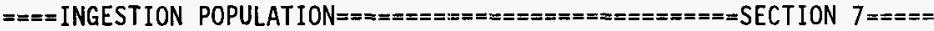
Atmospheric production definition (select option):

0 -Use food-weighted chi/Q, (food-sec/m3), enter value on this 1 ine 1-Use population-weighted chi $/ Q$

2-Use uniform production

3-Use chi/Q and production grids (PRODUCTION will be overridden)

Population ingesting aquatic foods, 0 defaults to total (person)

Population ingesting drinking water, 0 defaults to total (person)

Consider dose from food exported out of region (default=F)

Note below: $S^{*}$ or Source: 0-none, 1-ground water, 2-surface water 3-Derived concentration entered above

$=== \pm$ AQUATIC FOODS / DRINKING WATER INGESTION $========$ SECTION $8====$

Salt water? (default is fresh)

\begin{tabular}{|c|c|c|c|c|c|c|}
\hline $\begin{array}{l}\text { USE } \\
? \\
\text { T/F }\end{array}$ & $\begin{array}{l}\text { FOOD } \\
\text { TYPE }\end{array}$ & $\begin{array}{l}\text { TRAN- } \\
\text { SIT } \\
\mathrm{hr}\end{array}$ & $\begin{array}{l}\text { PROD- } \\
\text { UCTION } \\
\mathrm{kg} / \mathrm{yr}\end{array}$ & $\begin{array}{l}\text {-CONSUM } \\
\text { HOLDUP } \\
\text { da }\end{array}$ & $\begin{array}{l}\text { PION- } \\
\text { RATE } \\
\mathrm{kg} / \mathrm{yr}\end{array}$ & DRINKING WATER \\
\hline $\begin{array}{l}F \\
F \\
F\end{array}$ & $\begin{array}{l}\text { FISH } \\
\text { MOLLUS } \\
\text { CRUSTA } \\
\text { PLANTS }\end{array}$ & $\begin{array}{l}0.00 \\
0.00 \\
0.00 \\
0.00\end{array}$ & $\begin{array}{l}0.0 \mathrm{E}+00 \\
0.0 \mathrm{E}+00 \\
0.0 \mathrm{E}+00 \\
0.0 \mathrm{E}+00\end{array}$ & $\begin{array}{l}0.00 \\
0.00 \\
0.00 \\
0.00\end{array}$ & $\begin{array}{l}0.0 \\
0.0 \\
0.0 \\
0.0\end{array}$ & $\begin{array}{l}\text { Source (see above) } \\
\text { Treatment? T/F } \\
\text { HTdup/transit(da) } \\
\text { Consumpton (L/yr) }\end{array}$ \\
\hline
\end{tabular}

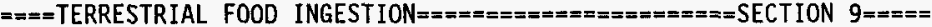

\begin{tabular}{|c|c|c|c|c|c|c|c|c|}
\hline USE & & GROW & --IRRIG & TION-- & & PROD- & -- CONS & PTION-- \\
\hline$\stackrel{?}{\mathrm{~T}} / \mathrm{F}$ & $\begin{array}{l}\text { FOOD } \\
\text { TYPE }\end{array}$ & $\begin{array}{l}\text { TIME } \\
\text { da }\end{array}$ & $\begin{array}{l}\text { S RATE } \\
\text { * in/yr }\end{array}$ & $\begin{array}{l}\text { TIME } \\
\text { mo/yr }\end{array}$ & $\begin{array}{l}\mathrm{YIELD} \\
\mathrm{kg} / \mathrm{m} 2\end{array}$ & $\begin{array}{l}\text { UCTION } \\
\mathrm{kg} / \mathrm{yr}\end{array}$ & $\begin{array}{l}\text { HOLDUP } \\
\mathrm{da}\end{array}$ & $\begin{array}{l}\text { RATE } \\
\mathrm{kg} / \mathrm{yr}\end{array}$ \\
\hline $\begin{array}{l}F \\
F \\
F\end{array}$ & $\begin{array}{l}\text { LEAF } V \\
\text { ROOT } V \\
\text { FRUIT } \\
\text { GRAIN }\end{array}$ & $\begin{array}{l}0.00 \\
0.00 \\
0.00 \\
0.00\end{array}$ & $\begin{array}{l}0.0 \\
0.0 \\
0.0 \\
0.0\end{array}$ & $\begin{array}{l}0.0 \\
0.0 \\
0.0 \\
0.0\end{array}$ & $\begin{array}{l}0.0 \\
0.0 \\
0.0 \\
0.0\end{array}$ & $\begin{array}{l}0.0 \mathrm{E}+00 \\
0.0 \mathrm{E}+00 \\
0.0 \mathrm{E}+00 \\
0.0 \mathrm{E}+00\end{array}$ & $\begin{array}{l}0.0 \\
0.0 \\
0.0 \\
0.0\end{array}$ & $\begin{array}{l}0.0 \\
0.0 \\
0.0 \\
0.0\end{array}$ \\
\hline
\end{tabular}

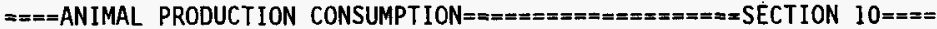


WHC-SD-WM-CN-016, Rev. 0

\begin{tabular}{|c|c|c|c|c|c|c|c|c|c|c|c|c|}
\hline \multirow[b]{2}{*}{$\begin{array}{l}\text { USE } \\
? \\
\text { T/F }\end{array}$} & \multirow[b]{2}{*}{$\begin{array}{l}\text { FOOD } \\
\text { TYPE }\end{array}$} & \multicolumn{2}{|c|}{---HUMAN---- } & \multirow{2}{*}{$\begin{array}{l}\text { TOTAL } \\
\text { PROD- } \\
\text { UCTION } \\
\mathrm{kg} / \mathrm{yr}\end{array}$} & \multirow{2}{*}{$\begin{array}{l}\text { DRINK } \\
\text { WATER } \\
\text { CONTAM } \\
\text { FRACT. }\end{array}$} & & \multicolumn{3}{|r|}{ FFFD } & \\
\hline & & $\begin{array}{l}\text { CONSUM } \\
\text { RATE } \\
\mathrm{kg} / \mathrm{yr}\end{array}$ & $\begin{array}{l}\text { IPTION } \\
\text { HOLDUP } \\
\text { da }\end{array}$ & & & $\begin{array}{l}\text { DIET } \\
\text { FRAC- } \\
\text { TION }\end{array}$ & $\begin{array}{c}\text { GROW } \\
\text { TIME } \\
\mathrm{da}\end{array}$ & & $\begin{array}{l}\text { RRIGA } \\
\text { RATE } \\
\text { in } / y r\end{array}$ & $\begin{array}{l}\text { TION-- } \\
\text { TIME } \\
\mathrm{mo} / \mathrm{yr}\end{array}$ & $\begin{array}{l}\text { YIELD } \\
\mathrm{kg} / \mathrm{m} 3\end{array}$ & $\begin{array}{l}\text { STOR- } \\
\text { AGE } \\
\text { da }\end{array}$ \\
\hline $\begin{array}{l}F \\
F \\
F \\
F\end{array}$ & $\begin{array}{l}\text { BEEF } \\
\text { POULTR } \\
\text { MILK } \\
\text { EGG }\end{array}$ & $\begin{array}{l}0.0 \\
0.0 \\
0.0 \\
0.0\end{array}$ & $\begin{array}{l}0.0 \\
0.0 \\
0.0 \\
0.0\end{array}$ & $\begin{array}{l}0.00 \\
0.00 \\
0.00 \\
0.00\end{array}$ & $\begin{array}{l}0.00 \\
0.00 \\
0.00 \\
0.00\end{array}$ & $\begin{array}{l}0.00 \\
0.00 \\
0.00 \\
0.00\end{array}$ & $\begin{array}{l}0.0 \\
0.0 \\
0.0 \\
0.0\end{array}$ & 0 & $\begin{array}{l}0.0 \\
0.0 \\
0.0 \\
0.0\end{array}$ & $\begin{array}{l}0.00 \\
0.00 \\
0.00 \\
0.00\end{array}$ & $\begin{array}{l}0.00 \\
0.00 \\
0.00 \\
0.00\end{array}$ & 0.0 \\
\hline & $\begin{array}{l}\text { BEEF } \\
\text { MILK }\end{array}$ & & & & & $\begin{array}{l}0.00 \\
0.00\end{array}$ & $\begin{array}{l}0.0 \\
0.0\end{array}$ & & $\begin{array}{l}.0 \\
.0\end{array}$ & $\begin{array}{l}0.00 \\
0.00\end{array}$ & $\begin{array}{l}0.00 \\
0.00\end{array}$ & $\begin{array}{l}0.0 \\
0.0\end{array}$ \\
\hline
\end{tabular}

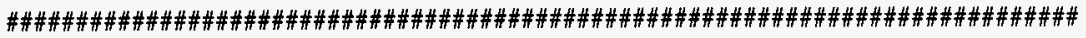


Case title: CWC - 12 percent nominal Pu-percent by weight-insoluble Pu. 1

Executed on: $02 / 28 / 96$ at $10: 20: 34$

Page A.

This is a far-field (wide-scale release, multiple site) scenario. Release is acute Individual dose

THE FOLLOWING TRANSPORT MODES ARE CONSIDERED Air

THE FOLLOWING EXPOSURE PATHS ARE CONSIDERED: Infinite plume, external Inhalation uptake

THE FOLLOWING TIMES ARE USED:
Intake ends after (yr):
1.0

Dose calculations ends after $(y r): \quad 50.0$

$=== \pm== \pm===$ FILENAMES AND TITLES OF FILES/LIBRARIES USED

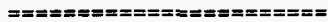

Input file name: IGENII $\backslash$ cwcpub. in

GENII Default Parameter Values (28-Mar-90 RAP)

Radionuclide Library - Times $<100$ years (23-JuTy-93 PDR)

External Dose Factors for GENII in person Sv/yr per Bq/n (8-May-90 R

PNL Solubilities, Yearly Dose Increments (23-Ju1-93 PDR)

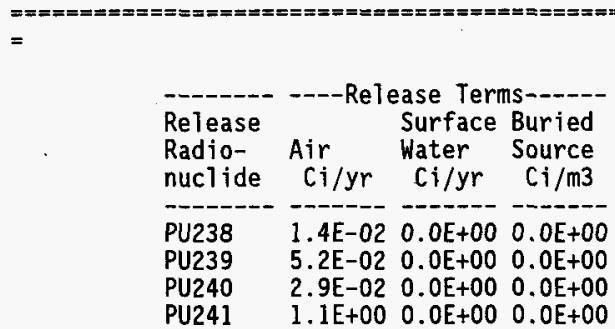


WHC-SD-WM-CN-016, Rev. 0

PU242 1.2E-06 $0.0 \mathrm{E}+00 \quad 0.0 \mathrm{E}+00$

AM241 $6.0 \mathrm{E}-02 \quad 0.0 \mathrm{E}+00 \quad 0.0 \mathrm{E}+00$

$== \pm=== \pm== \pm$ AIR TRANSPORT

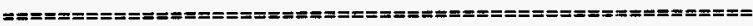

$1.0 E+00$ Input $E / Q$ value $(\mathrm{s} / \mathrm{m} 3)$

$==\pi==\pi==$ EXTERNAL EXPOSURE

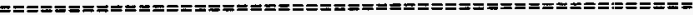

$1.0 \mathrm{E}+00$ Fraction of time spent in cloud

INHALATION

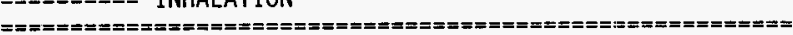

Resuspension not considered

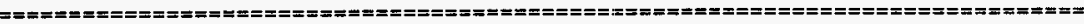

Input prepared by:

Date:

Input checked by:

Date:

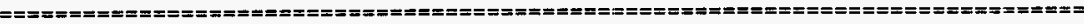


(Version 1.485 3-Dec-90)

Case title: CWC - 12 percent nominal Pu-percent by weight-insoluble Pu. Executed on: $02 / 28 / 96$ at $10: 20: 50$

Page C.

Acute release

Uptake/exposure period:

Dose commitment period:

Dose units:
1.0

50.0

Rem

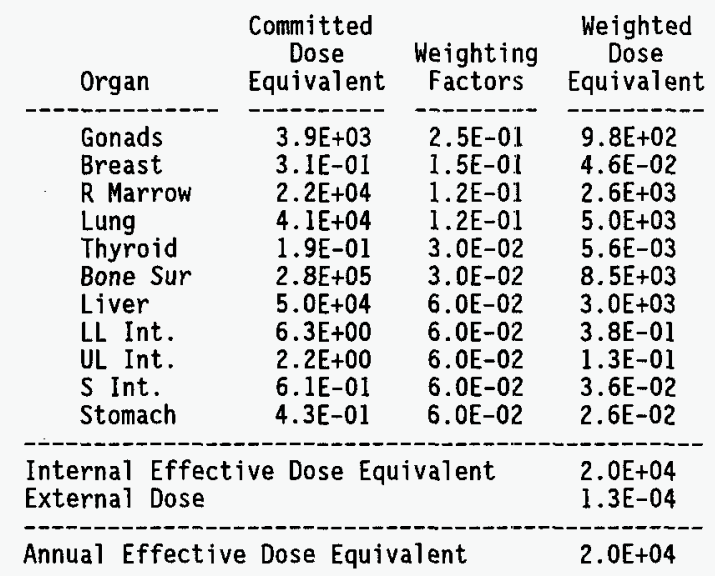

Controlling Organ:

Controlling Pathway:

Controlling Radionuclide:
Bone Sur

Inh

AM241 
WHC-SD-WM-CN-016, Rev. 0

Total Inhalation EDE:

2. $0 \mathrm{E}+04$

Total Ingestion EDE:

$0.0 \mathrm{E}+00$ 
WHC-SD-WM-CN-016, Rev. 0

\section{GENI I Dose Calculation Program}

(Version 1.485 3-Dec-90)

Case title: CWC - 12 percent nominal Pu-percent by weight-insoluble Pu. 2

Executed on: $02 / 28 / 96$ at $10: 20: 50$

Page C.

Acute release Uptake/exposure period: Dose commitment period:

Dose units:

Rem $\begin{array}{r}1.0 \\ 50.0\end{array}$

Dose Commitment Year

$1 \quad 2 \quad 3 \quad \ldots$

Internal : i

Intake : I

Year: 3

$0.0 \mathrm{E}+00 \quad \ldots$

I

$+$

$2 \mid 0: 0 E+00 \quad 0.0 E+00 \quad \ldots \quad$ Internal

i + + Effective

$1 \mid 1.3 \mathrm{E}+03+9.5 \mathrm{E}+02+7.9 \mathrm{E}+02+\ldots=2.0 \mathrm{E}+04$ Dose

Equivalent

II II II

Interna1

Cumulative

Annual

$1.3 \mathrm{E}+03+9.5 \mathrm{E}+02+7.9 \mathrm{E}+02+\ldots=2.0 \mathrm{E}+04$

Interna1

Dose

Dose

$+\quad+\quad+$

Externa1 
WHC-SD-WM-CN-016, Rev. 0

Annual $\quad 1.3 \mathrm{E}-04 \quad 0.0 \mathrm{E}+00 \quad 0.0 \mathrm{E}+00 \quad \ldots \quad 1.3 \mathrm{E}-04$

Dose

II II II Ii

Annual

Cumulative

Dose

$1.3 \mathrm{E}+03+9.5 \mathrm{E}+02+7.9 \mathrm{E}+02+\ldots$

2.0E+04 Dose

Maximum

1.3E+03 Annua 1

Dose Occurred

In Year 1 
Case title: CWC -12 percent nominal Pu-percent by weight-insoluble Pu. 3

Executed on: $02 / 28 / 96$ at $10: 20: 50$

Page $C$.

Acute release

Uptake/exposure period:

Dose commitment period:

Dose units:

1.0

50.0

Rem

Committed Dose Equivalent by Exposure Pathway

Pathway

Lung Stomach S Int. UL Int. LL Int. Bone Su R Marro

Testes

- - - - - - - - - -

Inhale

$3.9 \mathrm{E}+03$

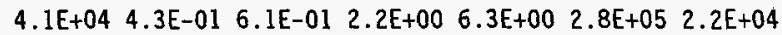

----o-

Total

$\begin{array}{llllll}4.1 \mathrm{E}+04 & 4.3 \mathrm{E}-01 \quad 6.1 \mathrm{E}-01 \quad 2.2 \mathrm{E}+00 \quad 6.3 \mathrm{E}+00 \quad 2.8 \mathrm{E}+05 \quad 2.2 \mathrm{E}+04\end{array}$

3. $9 \mathrm{E}+03$

\begin{tabular}{lllll} 
Pathway & Ovaries Muscle & Thyroid Liver \\
\hdashline Inhale & $3.9 \mathrm{E}+03$ & $3.1 \mathrm{E}-0 \mathrm{l}$ & $1.9 \mathrm{E}-01$ & $5.0 \mathrm{E}+04$ \\
\hdashline Total & $3.9 \mathrm{E}+03$ & $3.1 \mathrm{E}-01$ & $1.9 \mathrm{E}-01$ & $5.0 \mathrm{E}+04$
\end{tabular}

External Dose by Exposure Pathway

Pathway

\begin{tabular}{ll}
\hline Plume & $\overline{1.3 \mathrm{E}-04}$ \\
\hline Total & $\overline{1.3 \mathrm{E}-04}$
\end{tabular}


Case title: CWC - 12 percent nominal Pu-percent by weight-insoluble Pu. 4

Executed on: $02 / 28 / 96$ at $10: 20: 50$

Page C.

Acute release

Uptake/exposure period:

Dose commitment period:

Dose units:
1.0

50.0

Committed Dose Equivalent by Radionuclide

Radionuclide Lung Stomach S Int. UL Int. LL Int. Bone Su R Marro Testes

PU 240

4. $0 \mathrm{E}+02$

PU 238

1. $7 \mathrm{E}+02$

PU 242

$1.6 \mathrm{E}-02$

PU 241

$3.5 \mathrm{E}+02$

U 237

$0.0 \mathrm{E}+00$

AM 241

2. $3 \mathrm{E}+03$

PU 239

7. $2 \mathrm{E}+02$

Total

3. $9 \mathrm{E}+03$
1.1E+04 3.9E-02 7.5E-02 3.6E-01 1.1E+00 3.0E+04 2.3E+03

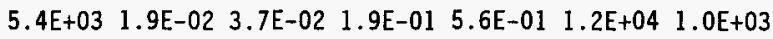

4.6E-01 1.6E-06 3.0E-06 1.5E-05 4.4E-05 1.1E+00 9.4E-02

$\begin{array}{lllllll}4.2 \mathrm{E}+03 & 4.0 \mathrm{E}-02 & 3.7 \mathrm{E}-02 & 1.1 \mathrm{E}-01 & 2.4 \mathrm{E}-01 & 2.4 \mathrm{E}+04 & 1.9 \mathrm{E}+03\end{array}$

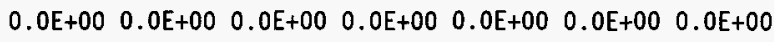

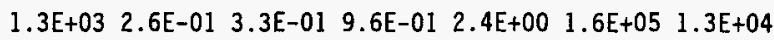

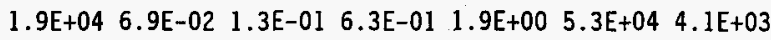

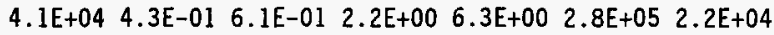

Radionuclide Ovaries Muscle Thyroid Liver

PU 240

PU 238

PU 242

PU 241

U 237
-

4.0E+02 $1.6 \mathrm{E}-02 \quad 1.3 \mathrm{E}-02 \quad 5.3 \mathrm{E}+03$

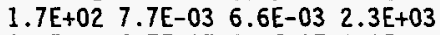

1.6E-02 6.7E-07 5.6E-07 2.1E-01

$3.4 \mathrm{E}+02 \quad 3.7 \mathrm{E}-02 \quad 1.6 \mathrm{E}-02 \quad 4.0 \mathrm{E}+03$

$0.0 \mathrm{E}+00 \quad 0.0 \mathrm{E}+00 \quad 0.0 \mathrm{E}+00 \quad 0.0 \mathrm{E}+00$ 
WHC-SD-WM-CN-016, Rev. 0

\begin{tabular}{|c|c|}
\hline $\begin{array}{l}\text { AM } 241 \\
\text { PU } 239\end{array}$ & $\begin{array}{llll}2.2 \mathrm{E}+03 & 2.2 \mathrm{E}-01 & 1.3 \mathrm{E}-01 & 2.9 \mathrm{E}+04 \\
7.2 \mathrm{E}+02 & 2.5 \mathrm{E}-02 & 2.4 \mathrm{E}-02 & 9.5 \mathrm{E}+03\end{array}$ \\
\hline Total & $3.9 \mathrm{E}+03$ 3.1E-01 $1.9 \mathrm{E}-01 \quad 5.0 \mathrm{E}+04$ \\
\hline
\end{tabular}




\section{GENII Dose Calculation Program}

(Version 1.485 3-Dec-90)

Case title: CWC - 12 percent nominal Pu-percent by weight-insoluble Pu. 5

Executed on: $02 / 28 / 96$ at $10: 20: 50$

Page C.

Acute release

Uptake/exposure period:

Dose commitment period:

Dose units:

\begin{tabular}{lcc} 
Radio- \\
nuclide & $\begin{array}{c}\text { Inhalation } \\
\text { Effective } \\
\text { Dose } \\
\text { Equivalent }\end{array}$ & $\begin{array}{c}\text { Ingestion } \\
\text { Effective } \\
\text { Dose } \\
\text { Equivalent }\end{array}$ \\
\hline PU 240 & $2.9 E+03$ & $0.0 E+00$ \\
PU 238 & $1.3 E+03$ & $0.0 E+00$ \\
PU 242 & $1.2 E-01$ & $0.0 E+00$ \\
PU 241 & $1.8 E+03$ & $0.0 E+00$ \\
U 237 & $0.0 E+00$ & $0.0 E+00$ \\
AM 241 & $8.9 E+03$ & $0.0 E+00$ \\
PU 239 & $5.2 E+03$ & $0.0 E+00$ \\
\hline
\end{tabular}

\begin{tabular}{c} 
External \\
Dose \\
$-8.9 \mathrm{E}-07$ \\
$4.4 \mathrm{E}-07$ \\
$0.0 \mathrm{E}+00$ \\
$8.4 \mathrm{E}-11$ \\
$0.0 \mathrm{E}+00$ \\
$1.3 \mathrm{E}-04$ \\
$1.1 \mathrm{E}-06$ \\
\hline
\end{tabular}

1.0

50.0

Rem

\begin{tabular}{cc}
$\begin{array}{c}\text { Internal } \\
\text { Effective } \\
\text { Dose } \\
\text { Equivalent }\end{array}$ & $\begin{array}{c}\text { Annual } \\
\text { Effective } \\
\text { Dose } \\
\text { Equivalent }\end{array}$ \\
\hline $2.9 \mathrm{E}+03$ & $2.9 \mathrm{E}+03$ \\
$1.3 \mathrm{E}+03$ & $1.3 \mathrm{E}+03$ \\
$1.2 \mathrm{E}-01$ & $1.2 \mathrm{E}-01$ \\
$1.8 \mathrm{E}+03$ & $1.8 \mathrm{E}+03$ \\
$0.0 \mathrm{E}+00$ & $0.0 \mathrm{E}+00$ \\
$8.9 \mathrm{E}+03$ & $8.9 \mathrm{E}+03$ \\
$5.2 \mathrm{E}+03$ & $5.2 \mathrm{E}+03$ \\
\hline
\end{tabular}


WHC-SD-WM-CN-016, Rev. 0

\#\#\#\#\#\#\#\#\#\#\#\#\#\#\#\#\#\#\#\# Program GENII Input File \#\#\#\#\#\#\#\#\#\# 8 Jui 88 \#\#\# Title: CWC - 23.9 percent nominal Pu-percent by weight-soluble Pu.

$\backslash$ GENII \cwcpu2 : in

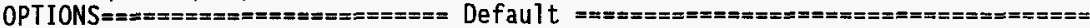

F Near-field scenario? (Far-field)

F Population dose? (Individual)

$\mathrm{T}$ Acute release?

Maximum Individual data set used

(Chronic)

Complete

TRANSPORT OPTIONS $============$ Section

$T$ Air Transport

F Surface Water Transport

F Biotic Transport (near-field) 3,4

$F$ Waste Form Degradation (near) 3,4

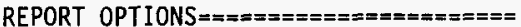

$T$ Report AEDE on 1y

$T$ Report by radionuclide

$T$ Report by exposure pathway

$F$ Debug report on screen
NEAR-FIELD: narrowly-focused

release, single site

FAR-FIELD: wide-scale release, muttiple sites

Complete

EXPOSURE PATHWAY OPTIONS $=====$ Section

$F$ Finite plume, external 5

$T$ Infinite plume, external 5

$F$ Ground, external

F Recreation, external

$T$ Inhalation uptake

$F$ Drinking water ingestion 7,8

$F$ Aquatic foods ingestion 7,8

F Terrestrial foods ingestion 7,9

F Animal product ingestion 7,10

$F$ Inadvertent soil ingestion

\section{INVENTORY \#\#\#\#\#\#\#\#\#\#\#\#\#\#\#\#\#\#\#\#\#\#\#\#\#\#\#\#\#\#\#\#\#\#\#\#\#\#\#\#\#\#\#\#\#\#\#\#\#\#\#\#\#\#\#\#\#\#\#\#\#\#\#\#\#}

4 Inventory input activity units: (1-pCi 2-uCi 3-mCi 4-Ci 5-Bq)

0 Surface soil source units $\left(1-\mathrm{m}_{2} 2-\mathrm{m} 33-\mathrm{kg}\right)$

Equilibrium question goes here

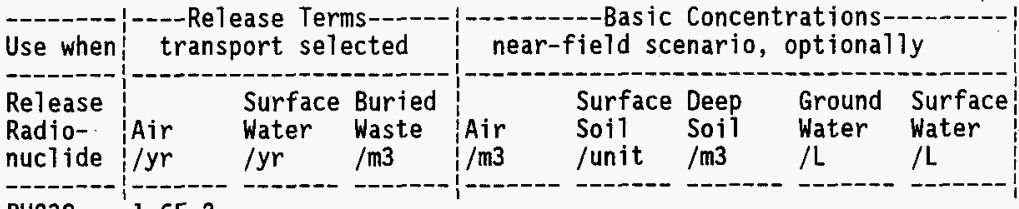

PU238 $1.6 \mathrm{E}-2$

PU239 4.3E-2

PU240 5.4E-2

PU241 1.7E+0

PU242 5.1E-6

AM241 8.8E-2

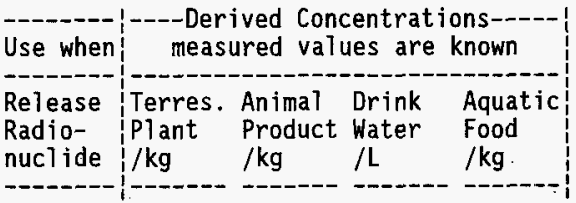

\section{TIME \#\#\#\#\#\#\#\#\#\#\#\#\#\#\#\#\#\#\#\#\#\#\#\#\#\#\#\#\#\#\#\#\#\#\#\#\#\#\#\#\#\#\#\#\#\#\#\#\#\#\#\#\#\#\#\#\#\#\#\#\#\#\#\#\#\#\#\#\#\#\#\#\#}

$1 \quad$ Intake ends after (yr)

50 Dose calc. ends after (yr)

0 Release ends after (yr) 
0 No. of years of air deposition prior to the intake period

0 No. of years of irrigation water deposition prior to the intake period

FAR-FIELD SCENARIOS (IF POPULATION DOSE) \#\#\#\#\#\#\#\#\#\#\#\#\#\#\#\#\#\#\#\#\#\#\#\#\#\#\#\#\#\#\#\#\#\#

0 Definition option: 1-Use population grid in file POP. IN

$0 \quad 2$-Use total entered on this line

NEAR-FIELD SCENARIOS \#\#\#\#\#\#\#\#\#\#\#\#\#\#\#\#\#\#\#\#\#\#\#\#\#\#\#\#\#\#\#\#\#\#\#\#\#\#\#\#\#\#\#\#\#\#\#\#\#\#\#\#\#\#

Prior to the beginning of the intake period: (yr)

When was the inventory disposed? (Package degradation starts)

When was LOIC? (Biotic transport starts)

Fraction of roots in upper soil (top $15 \mathrm{~cm}$ )

Fraction of roots in deep soil

Manual redistribution: deep soil/surface soil dilution factor

Source area for external dose modification factor (m2)

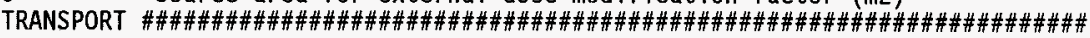

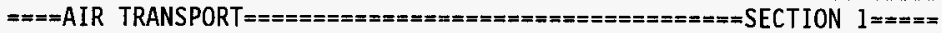

0 -Calculate PM

Option: 1-Use chi/Q or

(- Value

10

2-Select MI dist \& dir 0

3-Specify MI dist \& dir

Chi/Q or PM value

1

MI sector index $(1=S)$

MI distance from release point (m) 0

Release type $(0-3)$

Stack release ( $T / F)$

Stack height (m)

stack flow $(\mathrm{m} 3 / \mathrm{sec})$

Stack radius (m)

Effluent temp. (C)

Use jf data, (T/F) else chi/Q gridio

Building $x$-section (m2)

Building height (m)

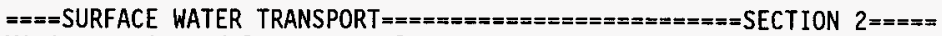
Mixing ratio model: 0 -use value, 1-river, 2-lake

Mixing ratio, dimensionless

Average river flow rate for: MIXFLG $=0(\mathrm{~m} 3 / \mathrm{s}), \operatorname{MIXFLG}=1,2(\mathrm{~m} / \mathrm{s})$, Transit time to irrigation withdrawl location (hr)

If mixing ratio model > 0 :

Rate of effluent discharge to receiving water body $(\mathrm{m} 3 / \mathrm{s})$

Longshore distance from release point to usage location (m)

offshore distance to the water intake (m)

Average water depth in surface water body $(\mathrm{m})$

Average river width (m), MIXFLG=1 only

Depth of effluent discharge point to surface water (m), lake only

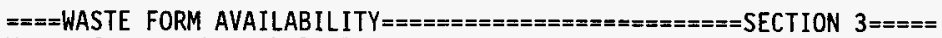
Waste form/package half life, (yr)

Waste thickness, (m)

Depth of soil overburden, $m$

$====$ BIOTIC TRANSPORT OF BURIED SOURCE $===============$ SECTION $4=====$ Consider during inventory decay/buildup period (T/F)?

Consider during intake period $(T / F)$ ? | l-Arid non agricultural

Pre-Intake site condition............. 2-Humid non agricultural 3-Agricultural 
$====$ EXTERNAL EXPOSURE

Exposure time:

Plume (hr)

Soil contamination (hr)

Swimming (hr)

Boating (hr)

Shoreline activities (hr)

Shoreline type: (1-river, 2-lake, 3-ocean, 4-tidal basin)

0

Transit time for release to reach aquatic recreation (hr)

Average fraction of time submersed in acute cloud (hr/person hr)

8766.0

0

0

0

0

0

0

F

$\mathrm{F}$

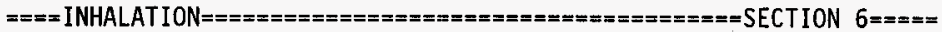
Hours of exposure to contamination per year

0-No resus- I-Use Mass Loading 2-Use Anspaugh model pension Mass loading factor $(\mathrm{g} / \mathrm{m} 3)$ Top soil available (cm)

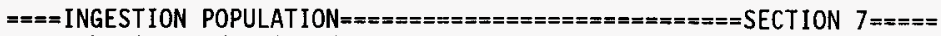
Atmospheric production definition (select option):

0 -Use food-weighted $\mathrm{chi} / \mathrm{Q},($ food-sec $/ \mathrm{m} 3)$, enter value on this line 1-Use population-weighted chi/Q

2-Use uniform production

3-Use chi/Q and production grids (PRODUCTION will be overridden)

Population ingesting aquatic foods, 0 defaults to total (person)

Population ingesting drinking water, 0 defaults to total (person)

Consider dose from food exported out of region (default=F)

Note below: $S^{*}$ or Source: 0-none, 1-ground water, 2-surface water 3-Derived concentration entered above $===$ AQUATIC FOODS / DRINKING WATER INGESTION $========$ SECTION $8====$

Salt water? (default is fresh)

\begin{tabular}{|c|c|c|c|c|c|c|c|}
\hline $\begin{array}{l}\text { USE } \\
? \\
\text { T/F }\end{array}$ & $\begin{array}{l}\text { FOOD } \\
\text { TYPE }\end{array}$ & $\begin{array}{l}\text { TRAN- } \\
\text { SIT } \\
\text { hr }\end{array}$ & $\begin{array}{l}\text { PROD- } \\
\text { UCTION } \\
\mathrm{kg} / \mathrm{yr}\end{array}$ & $\begin{array}{l}\text {-CONSUM } \\
\text { HOLDUP } \\
\text { da }\end{array}$ & $\begin{array}{l}\text { TION- } \\
\text { RATE } \\
\mathrm{kg} / \mathrm{yr}\end{array}$ & & DRINKING WATER \\
\hline $\begin{array}{l}F \\
F \\
F\end{array}$ & $\begin{array}{l}\text { FISH } \\
\text { MOLLUS } \\
\text { CRUSTA } \\
\text { PLANTS }\end{array}$ & $\begin{array}{l}0.00 \\
0.00 \\
0.00 \\
0.00\end{array}$ & $\begin{array}{l}0.0 \\
0.0 \\
0.0 \\
0.0\end{array}$ & $\begin{array}{l}0.00 \\
0.00 \\
0.00 \\
0.00\end{array}$ & $\begin{array}{l}0.0 \\
0.0 \\
0.0 \\
0.0\end{array}$ & $\begin{array}{l}0 \\
\mathrm{~T} \\
0 \\
0\end{array}$ & $\begin{array}{l}\text { Source(see above) } \\
\text { Treatment? T/F } \\
\text { Hldup/transit (da) } \\
\text { Consumpton (L/yr) }\end{array}$ \\
\hline
\end{tabular}

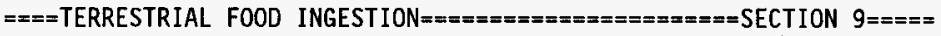

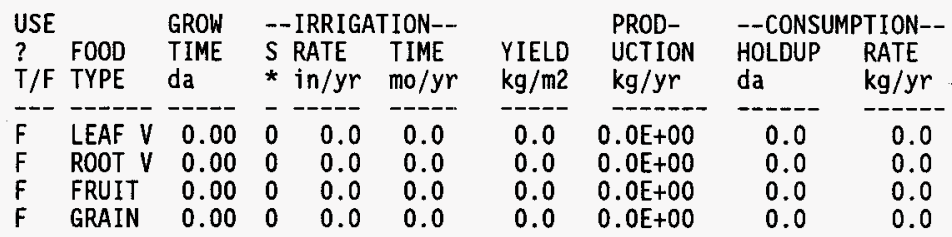

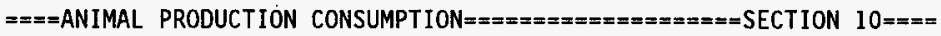


WHC-SD-WM-CN-016, Rev. 0

\begin{tabular}{|c|c|c|c|c|c|c|c|c|c|c|c|}
\hline $\begin{array}{l}\text { USE } \\
\stackrel{?}{T} / F\end{array}$ & $\begin{array}{l}\text { FOOD } \\
\text { TYPE }\end{array}$ & $\begin{array}{l}---H U M \\
\text { CONSUM } \\
\text { RATE } \\
\mathrm{kg} / \mathrm{yr}\end{array}$ & $\begin{array}{c}\text { MAN--.-- } \\
\text { MPTION } \\
\text { HOLDUP } \\
\text { da }\end{array}$ & $\begin{array}{l}\text { TOTAL } \\
\text { PROD- } \\
\text { UCTION } \\
\mathrm{kg} / \mathrm{yr}\end{array}$ & $\begin{array}{l}\text { DRINK } \\
\text { WATER } \\
\text { CONTAM } \\
\text { FRACT. }\end{array}$ & $\begin{array}{l}\text { DIET } \\
\text { FRAC- } \\
\text { TION }\end{array}$ & $\begin{array}{c}\text { GROW } \\
\text { TIME } \\
\text { da }\end{array}$ & $\begin{array}{l}\text {--STORED } \\
\text {-IRRIGA } \\
\text { S RATE } \\
* \text { in } / y r\end{array}$ & $\begin{array}{l}\text { FEED-- } \\
\text { TION-- } \\
\text { TIME } \\
\text { mo/yr }\end{array}$ & $\begin{array}{l}\text { YIELD } \\
\mathrm{kg} / \mathrm{m} 3\end{array}$ & $\begin{array}{l}\text { STOR- } \\
\text { AGE } \\
\text { da }\end{array}$ \\
\hline $\begin{array}{l}F \\
F\end{array}$ & $\begin{array}{l}\text { BEEF } \\
\text { POULTR } \\
\text { MILK } \\
\text { EGG }\end{array}$ & $\begin{array}{l}0.0 \\
0.0 \\
0.0 \\
0.0\end{array}$ & $\begin{array}{l}0.0 \\
0.0 \\
0.0 \\
0.0\end{array}$ & $\begin{array}{l}0.00 \\
0.00 \\
0.00 \\
0.00\end{array}$ & $\begin{array}{l}0.00 \\
0.00 \\
0.00 \\
0.00\end{array}$ & $\begin{array}{l}0.00 \\
0.00 \\
0.00 \\
0.00\end{array}$ & $\begin{array}{l}0.0 \\
0.0 \\
0.0 \\
0.0\end{array}$ & $\begin{array}{l}0.0 \\
0.0 \\
0.0 \\
0.0\end{array}$ & $\begin{array}{l}0.00 \\
0.00 \\
0.00 \\
0.00\end{array}$ & $\begin{array}{l}0.00 \\
0.00 \\
0.00 \\
0.00\end{array}$ & $\begin{array}{l}0.0 \\
0.0 \\
0.0 \\
0.0\end{array}$ \\
\hline & $\begin{array}{l}\text { BEEF } \\
\text { MILK }\end{array}$ & & & & & $\begin{array}{l}0.00 \\
0.00\end{array}$ & $\begin{array}{l}0.0 \\
0.0\end{array}$ & $\begin{array}{l}0.0 \\
0.0\end{array}$ & $\begin{array}{l}0.00 \\
0.00\end{array}$ & $\begin{array}{l}0.00 \\
0.00\end{array}$ & $\begin{array}{l}0.0 \\
0.0\end{array}$ \\
\hline
\end{tabular}

\#\#\#\#\#\#\#\#\#\#\#\#\#\#\#\#\#\#\#\#\#\#\#\#\#\#\#\#\#\#\#\#\#\#\#\#\#\#\#\#\#\#\#\#\#\#\#\#\#\#\#\#\#\#\#\#\#\#\#\#\#\#\#\#\#\#\#\#\#\#\#\#\#\#\# 
WHC-SD-WM-CN-016, Rev. 0

GENII Dose Calculation Program

(Version 1.485 3-Dec-90)

Case title: CWC - 23.9 percent nominal Pu-percent by weight-soluble Pu. 1

Executed on: $02 / 28 / 96$ at $10: 26: 38$

Page A.

This is a far-field (wide-scale release, multiple site) scenario.

Release is acute

Individua 7 dose

THE FOLLOWING TRANSPORT MODES ARE CONSIDERED

Air

THE FOLLOWING EXPOSURE PATHS ARE CONSIDERED:

Infinite plume, externat

Inhalation uptake

THE FOLLOWING TIMES ARE USED:

Intake ends after $(y r): \quad 1.0$

Dose calculations ends after $(y r): \quad 50.0$

$=========$ FILENAMES AND TITLES OF FILES/LIBRARIES USED

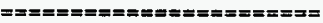

Input file name: IGENII \CwCpu2. in

GENII Default Parameter Values (28-Mar-90 RAP)

Radionuclide Library - Times $<100$ years (23-July-93 PDR)

External Dose Factors for GENII in person Sv/yr per Bq/n (8-May-90 $R$ Worst-Case Solubilities, YearTy Dose Increments (23-Ju1-93 PDR)

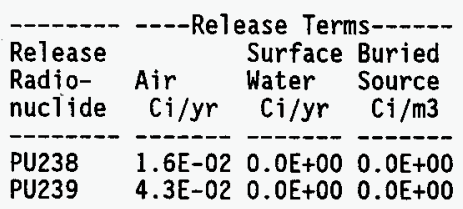


WHC-SD-WM-CN-016, Rev, 0

PU240 5.4E-02 $0.0 \mathrm{E}+00 \quad 0.0 \mathrm{E}+00$

PU241 $\quad 1.7 \mathrm{E}+00 \quad 0.0 \mathrm{E}+00 \quad 0.0 \mathrm{E}+00$

PU242 5.1E-06 $0.0 \mathrm{E}+00 \quad 0.0 \mathrm{E}+00$

AM241 8.8E-02 $0.0 \mathrm{E}+00 \quad 0.0 \mathrm{E}+00$

$=x=x=x=s=m$ AIR TRANSPORT

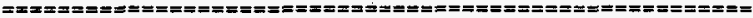

$1.0 \mathrm{E}+00$ Input $\mathrm{E} / \mathrm{Q}$ value $(\mathrm{s} / \mathrm{m} 3)$

$==0=m=s=s=$ EXTERNAL EXPOSURE

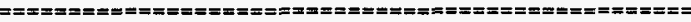

1.OE+00 Fraction of time spent in cloud

$=\pi=\pi=x==\approx$ INHALATION

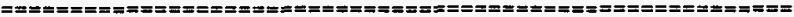

Resuspension not considered

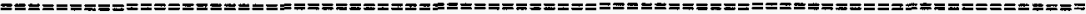

$=$

Input prepared by:

Date:

Input checked by:

Date:

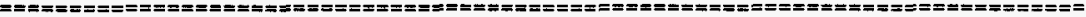


WHC-SD-WM-CN-016, Rev. 0

\section{GENII Dose Calculation Program}

(Version 1.485 3-Dec-90)

Case title: CWC - 23.9 percent nominal Pu-percent by weight-soluble Pu.

Executed on: $02 / 28 / 96$ at $10: 26: 55$

Page C.

Acute release

Uptake/exposure period:

Dose commitment period:

Dose units:

1.0

50.0

Rem

\begin{tabular}{|c|c|c|c|}
\hline Organ & $\begin{array}{l}\text { Committed } \\
\text { Dose } \\
\text { Equivalent }\end{array}$ & $\begin{array}{l}\text { Weighting } \\
\text { Factors }\end{array}$ & $\begin{array}{l}\text { Weighted } \\
\text { Dose } \\
\text { Equivalent }\end{array}$ \\
\hline $\begin{array}{l}\text { Gonads } \\
\text { Breast } \\
\text { R Marrow } \\
\text { Lung } \\
\text { Thyroid } \\
\text { Bone Sur } \\
\text { Liver } \\
\text { LL Int. } \\
\text { UL Int. } \\
\text { S Int. } \\
\text { Stomach }\end{array}$ & $\begin{array}{l}8.7 E+03 \\
5.4 E-01 \\
4.8 E+04 \\
4.3 E+03 \\
3.4 E-01 \\
6.2 E+05 \\
1.1 E+05 \\
7.7 E+00 \\
3.0 E+00 \\
9.0 E-01 \\
6.6 E-01\end{array}$ & $\begin{array}{l}2.5 \mathrm{E}-01 \\
1.5 \mathrm{E}-01 \\
1.2 \mathrm{E}-01 \\
1.2 \mathrm{E}-01 \\
3.0 \mathrm{E}-02 \\
3.0 \mathrm{E}-02 \\
6.0 \mathrm{E}-02 \\
6.0 \mathrm{E}-02 \\
6.0 \mathrm{E}-02 \\
6.0 \mathrm{E}-02 \\
6.0 \mathrm{E}-02\end{array}$ & $\begin{array}{l}2.2 \mathrm{E}+03 \\
8.0 \mathrm{E}-02 \\
5.8 \mathrm{E}+03 \\
5.2 \mathrm{E}+02 \\
1.0 \mathrm{E}-02 \\
1.8 \mathrm{E}+04 \\
6.4 \mathrm{E}+03 \\
4.6 \mathrm{E}-01 \\
1.8 \mathrm{E}-01 \\
5.4 \mathrm{E}-02 \\
4.0 \mathrm{E}-02\end{array}$ \\
\hline \multicolumn{3}{|c|}{$\begin{array}{l}\text { Internal Effective Dose Equivalent } \\
\text { External Dose }\end{array}$} & $\begin{array}{l}3.3 E+04 \\
2.0 E-04\end{array}$ \\
\hline \multicolumn{3}{|c|}{ Annual Effective Dose Equivalent } & $3.3 E+04$ \\
\hline
\end{tabular}

Controlling Organ:

Controlling Pathway:
Bone Sur

Inh 
WHC-SD-WM-CN-016, Rev. 0

Controlling Radionuclide:

AM241

Total Inhalation EDE:

$3.3 \mathrm{E}+04$

Total Ingestion EDE:

$0.0 \mathrm{E}+00$ 


\section{GENII Dose Calculation Program}

(Version 1.485 3-Dec-90)

Case title: CWC - 23.9 percent nominal Pu-percent by weight-soluble Pu.

Executed on: $02 / 28 / 96$ at $10: 26: 55$

Page $C$. 2

Acute release

Uptake/exposure period:

Dose commitment period:

Dose units:

$\operatorname{Rem}^{1.0} 50.0$

Dose Commitment Year

$1 \quad 2 \quad 3 \quad \ldots$

Internal : |

Intake : I

Year: 3

$0.0 \mathrm{E}+00 \quad \ldots$

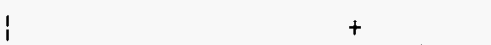

$2: 0.0 \mathrm{E}+00 \quad 0.0 \mathrm{E}+00 \quad \ldots \quad$ Internal

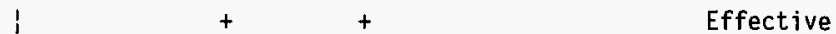

$1: 1.3 \mathrm{E}+03+8.9 \mathrm{E}+02+8.7 \mathrm{E}+02+\ldots=3.3 \mathrm{E}+04$ Dose

Equivalent

Internal

i) $11 \quad$ in

Annual

$1.3 E+03+8.9 E+02+8.7 E+02+\ldots=3.3 E+04$

Cumulative

Dose

Interna]

Dose

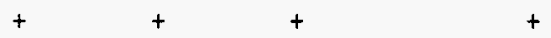

External 
WHC-SD-WM-CN-016, Rev. 0

$\begin{array}{llllll}\text { Annual } & 2.0 \mathrm{E}-04 & 0.0 \mathrm{E}+00 & 0.0 \mathrm{E}+00 & \ldots & 2.0 \mathrm{E}-04\end{array}$

Dose

II II $\quad$ II

Annual

Cumulative

Dose

$1.3 E+03+8.9 E+02+8.7 E+02+\ldots=3.3 E+04$ Dose

Maximum

1.3E+03 Annual

Dose 0ccurred

In Year 1 
WHC-SD-WM-CN-016, Rev. 0

\section{GENII Dose Calculation Program}

(Version 1.485 3-Dec-90)

Case title: CWC - 23.9 percent nominal Pu-percent by weight-soluble Pu. Executed on: $02 / 28 / 96$ at $10: 26: 55$

Page $C$. 3

Acute release

Uptake/exposure period:

Dose commitment period:

Dose units:

$$
\operatorname{Rem}^{1.0} \begin{array}{r}
50.0 \\
\end{array}
$$

Committed Dose Equivalent by Exposure Pathway

Pathway Lung Stomach S Int. UL Int. LL Int. Bone Su R Marro

Testes

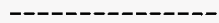

Inhale

$\begin{array}{lllll}4.3 \mathrm{E}+03 & 6.6 \mathrm{E}-01 & 9.0 \mathrm{E}-01 \quad 3.0 \mathrm{E}+00 & 7.7 \mathrm{E}+00 \quad 6.2 \mathrm{E}+05 \quad 4.8 \mathrm{E}+04\end{array}$

$8.7 E+03$

Total

$8.7 E+03$

$\begin{array}{lllll}4.3 \mathrm{E}+03 & 6.6 \mathrm{E}-01 & 9.0 \mathrm{E}-01 & 3.0 \mathrm{E}+00 \quad 7.7 \mathrm{E}+00 \quad 6.2 \mathrm{E}+05 \quad 4.8 \mathrm{E}+04\end{array}$

\begin{tabular}{lllll} 
Pathway & Ovaries Muscle & Thyroid Liver \\
\hline Inhale & $8.6 \mathrm{E}+03$ & $5.4 \mathrm{E}-01$ & $3.4 \mathrm{E}-01$ & $1.1 \mathrm{E}+05$ \\
\hline Tota 1 & $8.6 \mathrm{E}+03$ & $5.4 \mathrm{E}-01$ & $3.4 \mathrm{E}-01$ & $\mathrm{I} .1 \mathrm{E}+05$
\end{tabular}

External Dose by Exposure Pathway

Pathway

\begin{tabular}{ll} 
Plume & $-1.0 \mathrm{E}-04$ \\
\hline Total & $\mathbf{2 . 0 \mathrm { E } - 0 4}$
\end{tabular}




\section{GENII Dose Calculation Program}

(Version 1.485 3-Dec-90)

Case title: CWC - 23.9 percent nominal Pu-percent by weight-soluble Pu. 4

Executed on: $02 / 28 / 96$ at $10: 26: 55$

Page $C$.

Acute release Uptake/exposure period: Dose commitment period:

Dose units:

$$
\text { Rem } \begin{array}{r}
1.0 \\
50.0
\end{array}
$$

Committed Dose Equivalent by Radionuclide

Radionuclide Lung Stomach S Int. UL Int. LL Int. Bone Su R Marro Testes

PU 240

2. $0 \mathrm{E}+03$

PU 238

$5.2 E+02$

PU 242

$1.8 \mathrm{E}-01$

PU 241

$1.3 \mathrm{E}+03$

U 237

$0.0 \mathrm{E}+00$

AM 241

$3.3 \mathrm{E}+03$

PU 239

$1.6 \mathrm{E}+03$

Total

$8.7 \mathrm{E}+03$
$1.1 \mathrm{E}+03 \quad 1.0 \mathrm{E}-01 \quad 1.6 \mathrm{E}-01 \quad 6.5 \mathrm{E}-01 \quad 1.9 \mathrm{E}+00 \quad 1.4 \mathrm{E}+05 \quad 1.1 \mathrm{E}+04$

$\begin{array}{lllll}3.6 \mathrm{E}+02 & 3.1 \mathrm{E}-02 & 5.1 \mathrm{E}-02 & 2.2 \mathrm{E}-01 \quad 5.9 \mathrm{E}-01 \quad 3.8 \mathrm{E}+04 & 3.0 \mathrm{E}+03\end{array}$

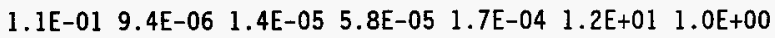

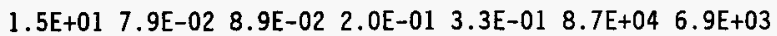

$\begin{array}{lllllll}0.0 E+00 & 0.0 E+00 & 0.0 E+00 & 0.0 E+00 & 0.0 E+00 & 0.0 E+00 & 0.0 E+00\end{array}$

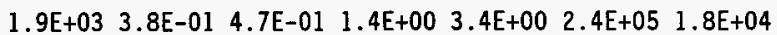

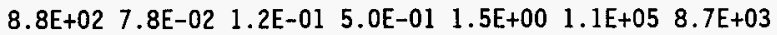

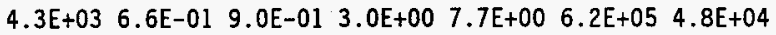

Radionuclide Ovaries Muscle Thyroid Liver

PU 240

PU 238

PU 242

PU 241

U 237

$\begin{array}{llll}2.0 \mathrm{E}+03 & 6.4 \mathrm{E}-02 & 6.1 \mathrm{E}-02 & 2.5 \mathrm{E}+04 \\ 5.1 \mathrm{E}+02 & 2.0 \mathrm{E}-02 & 1.9 \mathrm{E}-02 & 6.9 \mathrm{E}+03 \\ 1.8 \mathrm{E}-01 & 6.2 \mathrm{E}-06 & 5.6 \mathrm{E}-06 & 2.2 \mathrm{E}+00 \\ 1.3 \mathrm{E}+03 & 8.3 \mathrm{E}-02 & 3.5 \mathrm{E}-02 & 1.4 \mathrm{E}+04 \\ 0.0 \mathrm{E}+00 & 0.0 \mathrm{E}+00 & 0.0 \mathrm{E}+00 & 0.0 \mathrm{E}+00\end{array}$


WHC-SD-WM-CN-016, Rev. 0

AM 241

$3.2 \mathrm{E}+03 \quad 3.2 \mathrm{E}-01 \quad 1.8 \mathrm{E}-01 \quad 4.2 \mathrm{E}+04$

PU 239

1. $5 \mathrm{E}+03 \quad 4.8 \mathrm{E}-02 \quad 4.7 \mathrm{E}-02 \quad 2.0 \mathrm{E}+04$

Tota]

$8.6 E+03 \quad 5.4 E-01 \quad 3.4 E-01 \quad 1.1 E+05$ 
WHC-SD-WM-CN-016, Rev. 0

\section{GENII Dose Calculation Program}

(Version 1.485 3-Dec-90)

Case title: CWC - 23.9 percent nominal Pu-percent by weight-soluble Pu.

Executed on: 02/28/96 at 10:26:55 Page C. 5

Acute release

Uptake/exposure period:

Dose commitment period:

Dose units:

Inhalation Ingestion

Effective Effective

Radio-

nuclide

PU 240

PU 238

PU 242

PU 241

U 237

AM 241

PU 239
Dose

Equivalent Equivalent

$7.8 \mathrm{E}+03$

2. $1 \mathrm{E}+03$

6.9E-01

4. $6 \mathrm{E}+03$

$0.0 \mathrm{E}+00$

1. $3 \mathrm{E}+04$

6. $1 \mathrm{E}+03$
Dose

$0.0 E+00$

$0.0 \mathrm{E}+00$

$0.0 \mathrm{E}+00$

$0.0 \mathrm{E}+00$

$0.0 \mathrm{E}+00$

$0.0 \mathrm{E}+00$

$0.0 E+00$
External

Dose

$1.6 \mathrm{E}-06$

5. $1 \mathrm{E}-07$

$0.0 \mathrm{E}+00$

1. $3 \mathrm{E}-10$

$0.0 \mathrm{E}+00$

1. $9 \mathrm{E}-04$

9. $4 \mathrm{E}-07$
1.0

50.0

Rem
Internal Annual

Effective Effective Dose Dose Equivalent Equivalent

$7.8 \mathrm{E}+03 \quad 7.8 \mathrm{E}+03$

2. $1 E+03 \quad 2.1 E+03$

$6.9 \mathrm{E}-01 \quad 6.9 \mathrm{E}-01$

4. $6 \mathrm{E}+03 \quad 4.6 \mathrm{E}+03$

$0.0 \mathrm{E}+00 \quad 0.0 \mathrm{E}+00$

1. $3 \mathrm{E}+04 \quad 1.3 \mathrm{E}+04$

$6.1 E+03 \quad 6.1 E+03$ 
WHC-SD-WM-CN-016, Rev. 0

\#\#\#\#\#\#\#\#\#\#\#\#\#\#\#\#\#\#\#\#\# Program GENII Input File \#\#\#\#\#\#\#\#\#\#\# 8 Jul 88 \#\#\#\#

Title: CWC - 23.9 percent nominal Pu-percent by weight-insoluble Pu. $\backslash$ GENII $\backslash$ cWCpu2b. in

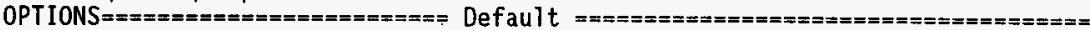

F Near-field scenario?

(Far-field)

(Individual)

(Chronic)

$\mathrm{T}$ Acute release?

Maximum Individual data set used

Complete

TRANSPORT OPTIONS $============$ Section

$T$ Air Transport

F Surface Water Transport

F Biotic Transport (near-field) 3,4

$F$ Waste Form Degradation (near) 3,4

REPORT OPTIONS $==================m= \pm==$

$T$ Report AEDE only

$T$ Report by radionuclide

$T$ Report by exposure pathway

$F$ Debug report on screen
NEAR-FIELD: narrowly-focused

release, single site

FAR-FIELD: wide-scale release, multiple sites

Complete

EXPOSURE PATHWAY OPTIONS $=====$ Section

$F$ Finite plume, external 5

$T$ Infinite plume, external 5

$F$ Ground, external

F Recreation, external

$T$ Inhalation uptake

$F$ Drinking water ingestion 7,8

$F$ Aquatic foods ingestion 7,8

F Terrestrial foods ingestion 7,9

F Animal product ingestion 7,10

F Inadvertent soil ingestion

\section{INVENTORY \#\#\#\#\#\#\#\#\#\#\#\#\#\#\#\#\#\#\#\#\#\#\#\#\#\#\#\#\#\#\#\#\#\#\#\#\#\#\#\#\#\#\#\#\#\#\#\#\#\#\#\#\#\#\#\#\#\#\#\#\#\#\#\#\#\#\#\#}

4 Inventory input activity units: (1-pCi $2-u C i \quad 3-m C i \quad 4-C i \quad 5-B q)$

0 Surface soil source units ( $1-\mathrm{m} 22-\mathrm{m} 33-\mathrm{kg}$ )

Equilibrium question goes here

\begin{tabular}{|c|c|c|c|c|c|c|c|}
\hline Use when & trans & ort selected & & ield sc & enario & optio & \\
\hline $\begin{array}{l}\text { Release } \\
\text { Radio- } \\
\text { nuclide }\end{array}$ & $\begin{array}{l}\text { Air } \\
/ / y r\end{array}$ & $\begin{array}{ll}\text { Surface } & \text { Buried } \\
\text { Water } & \text { Waste } \\
\text { /yr } & / \mathrm{m} 3\end{array}$ & $\left\{\begin{array}{l}\text { Air } \\
/ \mathrm{m} 3\end{array}\right.$ & $\begin{array}{l}\text { Surface } \\
\text { Soil } \\
\text { /unit }\end{array}$ & $\begin{array}{l}\text { Deep } \\
\text { SoiT } \\
/ \mathrm{m} 3\end{array}$ & $\begin{array}{l}\text { Ground } \\
\text { Water } \\
/ \mathrm{L}\end{array}$ & $\begin{array}{l}\text { Surface } \\
\text { Water } \\
\text { /L }\end{array}$ \\
\hline $\begin{array}{l}\text { PU238 } \\
\text { PU239 } \\
\text { PU240 } \\
\text { PU241 } \\
\text { PU242 } \\
\text { AM241 }\end{array}$ & $\begin{array}{l}1.6 \mathrm{E}-2 \\
4.3 \mathrm{E}-2 \\
5.4 \mathrm{E}-2 \\
1.7 \mathrm{E}+0 \\
5.1 \mathrm{E}-6 \\
8.8 \mathrm{E}-2\end{array}$ & & & & & & \\
\hline
\end{tabular}

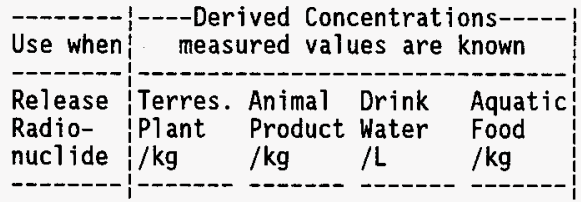

TIME \#\#\#\#\#\#\#\#\#\#\#\#\#\#\#\#\#\#\#\#\#\#\#\#\#\#\#\#\#\#\#\#\#\#\#\#\#\#\#\#\#\#\#\#\#\#\#\#\#\#\#\#\#\#\#\#\#\#\#\#\#\#\#\#\#\#\#\#\#\#

1 Intake ends after (yr)

50 Dose calc. ends after (yr)

0 . Release ends after (yr) 
WHC-SD-WM-CN-016, Rev. 0

0 No. of years of air deposition prior to the intake period

0 No. of years of irrigation water deposition prior to the intake period

FAR-FIELD SCENARIOS (IF POPULATION DOSE) \#\#\#\#\#\#\#\#\#\#\#\#\#\#\#\#\#\#\#\#\#\#\#\#\#\#\#\#\#\#\#\#\#\#

0 Definition option: 1-Use population grid in file POP. IN

0

2-Use total entered on this line

\section{NEAR-FIELD SCENARIOS \#\#\#\#\#\#\#\#\#\#\#\#\#\#\#\#\#\#\#\#\#\#\#\#\#\#\#\#\#\#\#\#\#\#\#\#\#\#\#\#\#\#\#\#\#\#\#\#\#\#\#\#\#\#\#\#\#}

Prior to the beginning of the intake period: $(y r)$

0

0

0

0

0

0

TRANSPORT
When was the inventory disposed? (Package degradation starts)

When was LOIC? (Biotic transport starts)

Fraction of roots in upper soil (top $15 \mathrm{~cm}$ )

Fraction of roots in deep soil

Manual redistribution: deep soil/surface soil dilution factor Source area for external dose modification factor (m2)

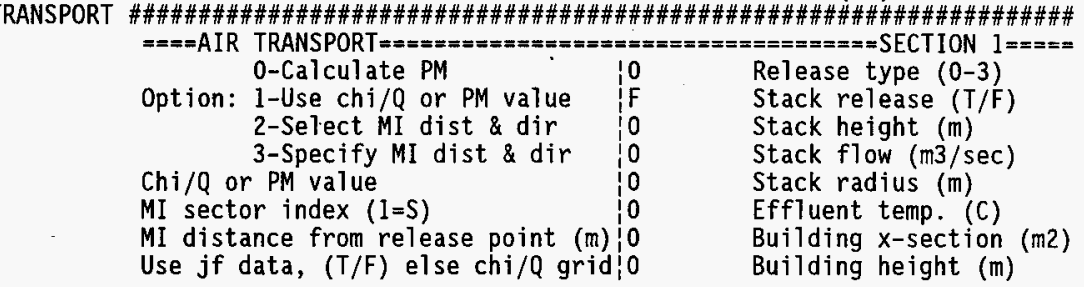

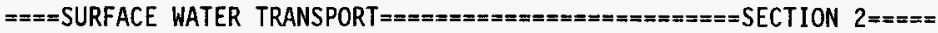
Mixing ratio model: 0-use value, 1-river, 2-1ake Mixing ratio, dimensionless

Average river flow rate for: MIXFLG=0 (m3/s), MIXFLG=1,2 (m/s), Transit time to irrigation withdrawl location (hr) If mixing ratio model $>0$ :

Rate of effluent discharge to receiving water body $(\mathrm{m} 3 / \mathrm{s})$ Longshore distance from release point to usage location (m) off shore distance to the water intake (m) Average water depth in surface water body (m) Average river width (m), MIXFLG=1 only Depth of effluent discharge point to surface water (m), lake only

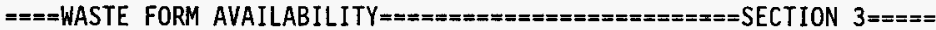
Waste form/package half life, (yr)

Waste thickness, (m)

Depth of soil overburden, m

$====$ BIOTIC TRANSPORT OF BURIED SOURCE $==============x=$ SECTION $4=====$ Consider during inventory decay/buildup period $(T / F)$ ?

Consider during intake period $(T / F)$ ? : 1-Arid non agricultural

Pre-Intake site condition. 2-Humid non agricuTtural 3-Agricultural 
$====$ EXTERNAL EXPOSURE

Exposure time:

Plume (hr)

Soil contamination (hr)

Swimming (hr)

Boating (hr)

Shoreline activities (hr)
Residential irrigation:

Consider: (T/F)

Source: 1-ground water

2-surface water

Application rate (in/yr)

Duration (mo/yr)

Shoreline type: (1-river, 2-1ake, 3-ocean, 4-tidal basin)

Transit time for release to reach aquatic recreation (hr)

Average fraction of time submersed in acute cloud (hr/person hr)

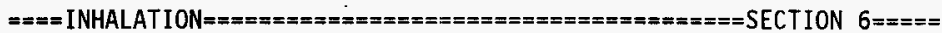

8766.0

Hours of exposure to contamination per year

0

0 -No resus- 1-Use Mass Loading

pension Mass loading factor $(\mathrm{g} / \mathrm{m} 3)$

2-Use Anspaugh mode1

0

$====$ INGESTION POPULATION $==x========================$ SECTION $7=====$ Atmospheric production definition (select option):

0 -Use food-weighted chi/Q, (food-sec/m3), enter-value on this line 1-Use population-weighted chi/Q

2-Use uniform production

3-Use chi/Q and production grids (PRODUCTION will be overridden)

Population ingesting aquatic foods, 0 defaults to total (person)

Population ingesting drinking water, 0 defaults to total (person)

Consider dose from food exported out of region (default=F)

Note below: $5^{*}$ or Source: 0-none, 1-ground water, 2-surface water 3-Derived concentration entered above

$==x=$ AQUATIC FOODS / DRINKING WATER INGESTION $========5$ SCTION $8====$

Salt water? (default is fresh)

\begin{tabular}{|c|c|c|c|c|c|}
\hline $\begin{array}{ll}\text { USE } & \\
? & \text { FOOD } \\
\text { T/F } & \text { TYPE }\end{array}$ & $\begin{array}{l}\text { TRAN- } \\
\text { SIT } \\
\text { hr }\end{array}$ & $\begin{array}{l}\text { PROD- } \\
\text { UCTION } \\
\mathrm{kg} / \mathrm{yr}\end{array}$ & $\begin{array}{l}\text {-CONSUMF } \\
\text { HOLDUP } \\
\mathrm{da}\end{array}$ & $\begin{array}{l}\text { PTION- } \\
\text { RATE } \\
\mathrm{kg} / \mathrm{yr}\end{array}$ & DRINKING WATER \\
\hline $\begin{array}{l}\text { FISH } \\
\text { MOLLUS } \\
\text { CRUSTA } \\
\text { PLANTS }\end{array}$ & $\begin{array}{l}0.00 \\
0.00 \\
0.00 \\
0.00\end{array}$ & $\begin{array}{l}0.0 E+00 \\
0.0 E+00 \\
0.0 E+00 \\
0.0 E+00\end{array}$ & $\begin{array}{l}0.00 \\
0.00 \\
0.00 \\
0.00\end{array}$ & $\begin{array}{l}0.0 \\
0.0 \\
0.0 \\
0.0\end{array}$ & $\begin{array}{l}\text { Source (see above) } \\
\text { Treatment? T/F } \\
\text { HIdup/transit (da) } \\
\text { Consumpton ( } \mathrm{L} / \mathrm{yr} \text { ) }\end{array}$ \\
\hline
\end{tabular}

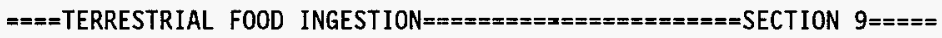

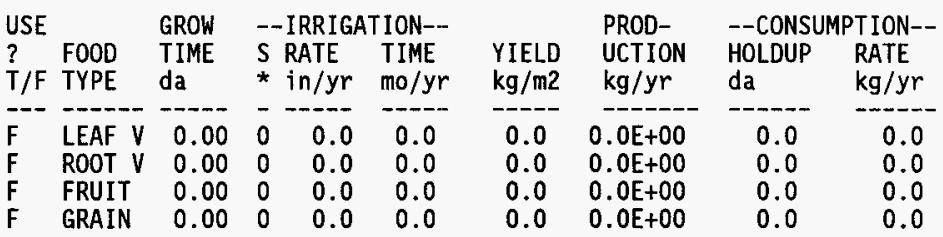

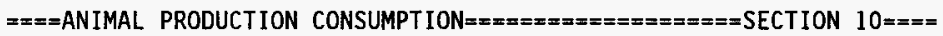


WHC-SD-WM-CN-016, Rev. 0

\begin{tabular}{|c|c|c|c|c|c|c|c|c|c|c|c|}
\hline \multirow{4}{*}{$\begin{array}{l}\text { USE } \\
\stackrel{?}{\mathrm{~T}} / \mathrm{F}\end{array}$} & \multirow{4}{*}{$\begin{array}{l}\text { FOOD } \\
\text { TYPE }\end{array}$} & \multirow{2}{*}{\multicolumn{2}{|c|}{$\begin{array}{l}--- \text { HUMAN---- } \\
\text { CONSUMPTION }\end{array}$}} & \multirow{4}{*}{$\begin{array}{l}\text { TOTAL } \\
\text { PROD- } \\
\text { UCTION } \\
\mathrm{kg} / \mathrm{yr}\end{array}$} & \multirow{4}{*}{$\begin{array}{l}\text { DRINK } \\
\text { WATER } \\
\text { CONTAM } \\
\text { FRACT. }\end{array}$} & \multicolumn{6}{|c|}{ 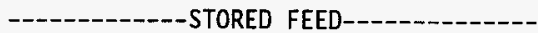 } \\
\hline & & & & & & DIET & GROW & -IRRIGA & TION-- & & STOR- \\
\hline & & RATE & HOLDUP & & & FRAC- & TIME & S RATE & TIME & YIELD & AGE \\
\hline & & $\mathrm{kg} / \mathrm{yr}$ & da & & & TION & da & * in/yr & mo/yr & $\mathrm{kg} / \mathrm{m} 3$ & $\mathrm{da}$ \\
\hline F & BEFF & 0.0 & 0.0 & 0.00 & 0.00 & 0.00 & 0.0 & 0.0 & 0.00 & 0.00 & 0.0 \\
\hline F & POULTR & 0.0 & 0.0 & 0.00 & 0.00 & 0.00 & 0.0 & 0.0 & 0.00 & 0.00 & 0.0 \\
\hline $\mathrm{F}$ & MILK & 0.0 & 0.0 & 0.00 & 0.00 & 0.00 & 0.0 & 0.0 & 0.00 & 0.00 & 0.0 \\
\hline $\mathrm{F}$ & $E G G$ & 0.0 & 0.0 & 0.00 & 0.00 & 0.00 & 0.0 & 0.0 & 0.00 & 0.00 & 0.0 \\
\hline & & & & & & & & & & & \\
\hline & BEEF & & & & & 0.00 & 0.0 & 0.0 & 0.00 & 0.00 & 0.0 \\
\hline & MILK & & & & & 0.00 & 0.0 & 0.0 & 0.00 & 0.00 & 0.0 \\
\hline
\end{tabular}




\section{GENII Dose Calculation Program}

(Version 1.485 3-Dec-90)

Case title: CWC - 23.9 percent nominal Pu-percent by weight-insoluble Pu Executed on: $02 / 28 / 96$ at $10: 31: 36$

Page A. 1

This is a far-field (wide-scale release, multiple site) scenario. Release is acute

Individual dose

THE FOLLOWING TRANSPORT MODES ARE CONSIDERED Air

THE FOLLOWING EXPOSURE PATHS ARE CONSIDERED: Infinite plume, external Inhalation uptake

THE FOLLOWING TIMES ARE USED:

Intake ends after $(y r):$

Dose calculations ends after ( $y r): \quad 50.0$

$=====s===$ FILENAMES AND TITLES OF FILES/LIBRARIES USED

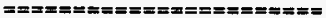

Input file name: IGENII\cwcpu2b. in

GENII Default Parameter Values (28-Mar-90 RAP)

Radionuclide Library - Times $<100$ years (23-July-93 PDR)

External Dose Factors for GENII in person SV/yr per Bq/n (8-May-90 R

PNL Solubilities, Yearly Dose Increments (23-Jul-93 PDR)

\begin{tabular}{|c|c|c|c|}
\hline $\begin{array}{l}\text { Release } \\
\text { Radio- } \\
\text { nuclide }\end{array}$ & $\begin{array}{l}\text { Air } \\
\mathrm{Ci} / \mathrm{yr}\end{array}$ & $\begin{array}{c}\text { Surface } \\
\text { Water } \\
\text { Ci/yr }\end{array}$ & $\begin{array}{c}\text { Buried } \\
\text { Source } \\
\mathrm{Ci} / \mathrm{m} 3\end{array}$ \\
\hline J23 & $\begin{array}{l}1.6 \mathrm{E}-02 \\
4.3 \mathrm{E}-02\end{array}$ & $\begin{array}{l}0.0 E+00 \\
0.0 E+00\end{array}$ & $\begin{array}{l}0.0 E+00 \\
0.0 E+00\end{array}$ \\
\hline
\end{tabular}


WHC-SD-WM-CN-016, Rev. 0

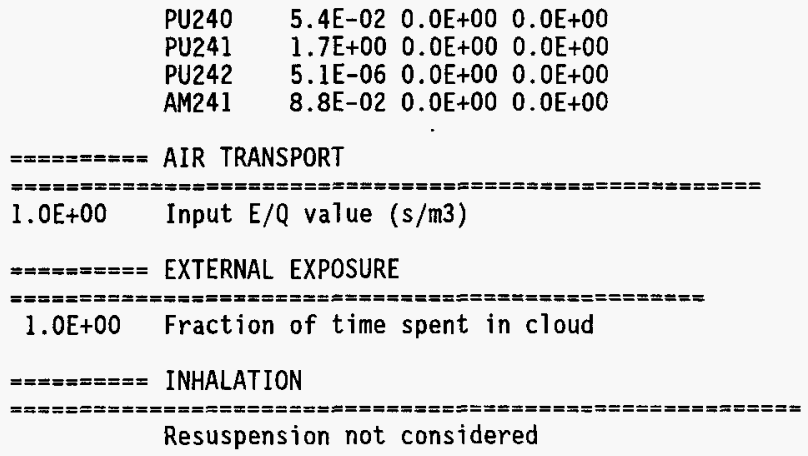

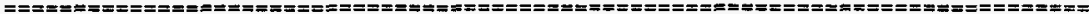

Input prepared by:

Date:

Input checked by:

Date:

=

$=$ 
WHC-SD-WM-CN-016, Rev. 0

GENII Dose CalcuTation Program

(Version 1.485 3-Dec-90)

Case title: CWC - 23.9 percent nominal Pu-percent by weight-insoluble Pu Executed on: $02 / 28 / 96$ at $10: 31: 53$

Page C.

Acute release

Uptake/exposure period:

Dose commitment period:

Dose units:

1.0

50.0

Rem

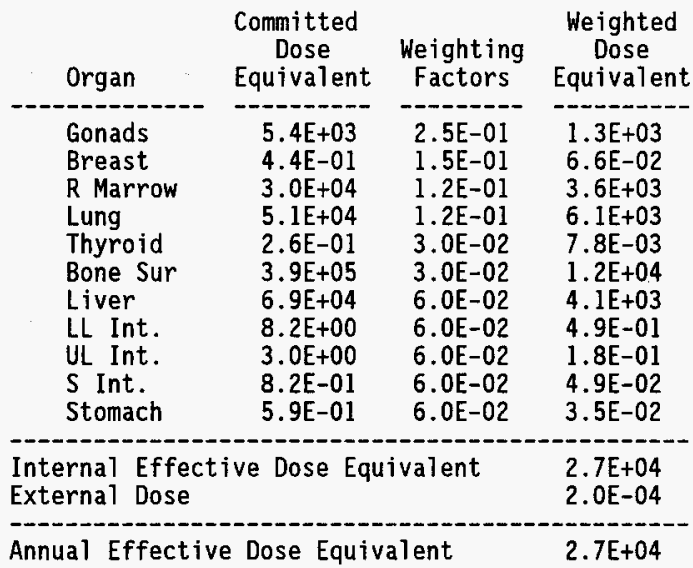

Controlling Organ:

Controlling Pathway:

Controlling Radionuclide:
Bone Sur

Inh

AM241 
WHC-SD-WM-CN-016, Rev. 0

Total Inhalation EDE:

$2.7 \mathrm{E}+04$

Total Ingestion EDE:

$0.0 \mathrm{E}+00$ 
WHC-SD-WM-CN-016, Rev. 0

\section{GENII Dose Calculation Program}

(Version 1.485 3-Dec-90)

Case title: CWC -23.9 percent nominal Pu-percent by weight-insoluble Pu

Executed on: $02 / 28 / 96$ at $10: 31: 53$

Page $C$. 2

Acute release

Uptake/exposure period:

Dose commitment period:

Dose units:

$$
\text { Rem } \begin{array}{r}
1.0 \\
50.0
\end{array}
$$

Dose Commitment Year

$1 \quad 2 \quad 3 \quad \ldots$

Internal : i

Intake : i

Year: 3

I

$0.0 E+00 \quad \ldots$

$+$

2

$0.0 \mathrm{E}+00$

$0.0 E+00$

Internal

i

$+$

$+$

Effective

$1: 1.7 \mathrm{E}+03+1.2 \mathrm{E}+03+1.0 \mathrm{E}+03+\ldots=2.7 \mathrm{E}+04$ Dose

Equivalent

i1 $11 \quad$ i1

Internal

Cumulative

Annual

$1.7 \mathrm{E}+03+1.2 \mathrm{E}+03$

$3+.0 \mathrm{E}+03+$

$+\ldots=2.7 \mathrm{E}+04$

Internal

Dose

Dose

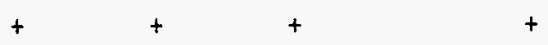

External 
WHC-SD-WM-CN-016, Rev. 0

Annual $\quad 2.0 \mathrm{E}-04 \quad 0.0 \mathrm{E}+00 \quad 0.0 \mathrm{E}+00 \quad \ldots \quad 2.0 \mathrm{E}-04$

Dose

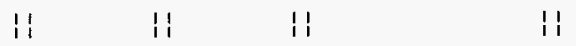

Annual

Dose

$1.7 \mathrm{E}+03$

$+1.2 \mathrm{E}+03+1.0 \mathrm{E}+03$

$+\ldots$

$=2.7 \mathrm{E}+04$

Cumulative

Maximum

1.7E+03 Annual

Dose 0ccurred

In Year 1

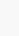




\section{GENI I Dose Calculation Program}

(Version 1.485 3-Dec-90)

Case title: CWC - 23.9 percent nominal Pu-percent by weight-insoluble $\mathrm{Pu}$ 3

Executed on: $02 / 28 / 96$ at $10: 31: 53$

Page $C$.

Acute release

Uptake/exposure period:

Dose commitment period:

Dose units:

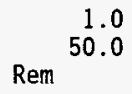

Committed Dose Equivalent by Exposure Pathway

Pathway Lung Stomach S Int. UL Int. LL Int. Bone Su R Marro Testes

-----

Inhale

$5.4 \mathrm{E}+03$

$\begin{array}{llllll}5.1 \mathrm{E}+04 & 5.9 \mathrm{E}-01 \quad 8.2 \mathrm{E}-01 \quad 3.0 \mathrm{E}+00 \quad 8.2 \mathrm{E}+00 \quad 3.9 \mathrm{E}+05 \quad 3.0 \mathrm{E}+04\end{array}$

Total

$\begin{array}{lllll}5.1 \mathrm{E}+04 \quad 5.9 \mathrm{E}-01 \quad 8.2 \mathrm{E}-01 \quad 3.0 \mathrm{E}+00 \quad 8.2 \mathrm{E}+00 \quad 3.9 \mathrm{E}+05 \quad 3.0 \mathrm{E}+04 & 4\end{array}$

$5.4 \mathrm{E}+03$

\begin{tabular}{|c|c|c|c|c|}
\hline thi & Ovaries & Muscle & Thyroid & Liver \\
\hline & $5.3 E+03$ & $4.4 \mathrm{E}-0 \mathrm{I}$ & $2.6 \mathrm{E}-01$ & $6.9 \mathrm{E}+04$ \\
\hline & 5.3 & 1 & $2.6 \mathrm{E}-01$ & 04 \\
\hline
\end{tabular}

External Dose by Exposure Pathway

Pathway

\begin{tabular}{ll}
\hline Plume & $\overline{2.0 \mathrm{E}-04}$ \\
\hline Total & $2.0 \mathrm{E}-04$
\end{tabular}




\section{GENII Dose Calculation Program}

(Version $1.4853-$ Dec-90)

Case title: CWC -23.9 percent nominal Pu-percent by weight-insoluble Pu 4

Executed on: $02 / 28 / 96$ at $10: 31: 53$

Page C.

Acute release

Uptake/exposure period:

Dose commitment period:

Dose units:
1.0

50.0

Rem

Committed Dose Equivalent by Radionuclide

Radionuclide Lung Stomach S Int. UL Int. LL Int. Bone Su R Marro Testes

PU 240
$7.6 E+02$
$P U 238$
$1.9 E+02$
$P U 242$
$6.7 E-02$
$P U 241$
$5.4 E+02$
$U \quad 237$
$0.0 E+00$
$A M 241$
$3.3 E+03$
$P U 239$
$5.9 E+02$

2.1E+04 7.3E-02 1.4E-01 6.7E-01 2.1E+00 5.6E+04 4.3E+03

$6.2 \mathrm{E}+03 \quad 2.2 \mathrm{E}-02 \quad 4.3 \mathrm{E}-02 \quad 2.2 \mathrm{E}-01 \quad 6.5 \mathrm{E}-01 \quad 1.4 \mathrm{E}+04 \quad 1.2 \mathrm{E}+03$

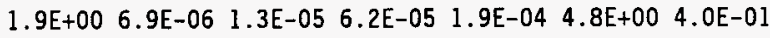

$\begin{array}{lllllll}6.5 \mathrm{E}+03 & 6.2 \mathrm{E}-02 & 5.8 \mathrm{E}-02 & 1.7 \mathrm{E}-01 & 3.7 \mathrm{E}-01 & 3.7 \mathrm{E}+04 & 2.9 \mathrm{E}+03\end{array}$

$\begin{array}{llllll}0.0 \mathrm{E}+00 & 0.0 \mathrm{E}+00 & 0.0 \mathrm{E}+00 & 0.0 \mathrm{E}+00 & 0.0 \mathrm{E}+00 \quad 0.0 \mathrm{E}+00 \quad 0.0 \mathrm{E}+00\end{array}$

$\begin{array}{llllllll}1.9 \mathrm{E}+03 & 3.8 \mathrm{E}-01 & 4.7 \mathrm{E}-01 & 1.4 \mathrm{E}+00 & 3.4 \mathrm{E}+00 & 2.4 \mathrm{E}+05 \quad 1.8 \mathrm{E}+04\end{array}$

$\begin{array}{llll}1.6 \mathrm{E}+04 & 5.7 \mathrm{E}-02 & 1.1 \mathrm{E}-01 \quad 5.2 \mathrm{E}-01 \quad 1.6 \mathrm{E}+00 \quad 4.4 \mathrm{E}+04 \quad 3.4 \mathrm{E}+03\end{array}$

Total

$\begin{array}{lllll}5.1 \mathrm{E}+04 & 5.9 \mathrm{E}-01 \quad 8.2 \mathrm{E}-01 \quad 3.0 \mathrm{E}+00 \quad 8.2 \mathrm{E}+00 \quad 3.9 \mathrm{E}+05 \quad 3.0 \mathrm{E}+04\end{array}$

$5.4 \mathrm{E}+03$

Radionuclide Ovaries Muscle Thyroid Liver

PU 240

PU 238

PU 242

PU 241

U 237
- Ovaries Muscle Thyroid Liver

$\begin{array}{llll}7.6 \mathrm{E}+02 & 2.9 \mathrm{E}-02 & 2.5 \mathrm{E}-02 & 1.0 \mathrm{E}+04\end{array}$

1. $9 \mathrm{E}+02 \quad 8.8 \mathrm{E}-03 \quad 7.6 \mathrm{E}-03 \quad 2.7 \mathrm{E}+03$

6.8E-02 2.8E-06 2.4E-06 9.0E-01

$5.3 \mathrm{E}+02 \quad 5.8 \mathrm{E}-02 \quad 2.5 \mathrm{E}-02 \quad 6.2 \mathrm{E}+03$

$0.0 \mathrm{E}+00 \quad 0.0 \mathrm{E}+00 \quad 0.0 \mathrm{E}+00 \quad 0.0 \mathrm{E}+00$ 
WHC-SD-WM-CN-016, Rev. 0

AM 241

$3.2 \mathrm{E}+03 \quad 3.2 \mathrm{E}-01 \quad 1.8 \mathrm{E}-01 \quad 4.2 \mathrm{E}+04$

PU 239

$5.9 \mathrm{E}+02 \quad 2.1 \mathrm{E}-02 \quad 2.0 \mathrm{E}-02 \quad 7.8 \mathrm{E}+03$

Total

$\begin{array}{llll}5.3 \mathrm{E}+03 \quad 4.4 \mathrm{E}-01 \quad 2.6 \mathrm{E}-01 \quad 6.9 \mathrm{E}+04 & 4\end{array}$ 
WHC-SD-WM-CN-016, Rev. 0

\section{GENII Dose Calculation Program}

(Version 1.485 3-Dec-90)

Case title: CWC - 23.9 percent nominal Pu-percent by weight-insoluble Pu

Executed on: $02 / 28 / 96$ at $10: 31: 53$

Page C.

5

Acute release

Uptake/exposure period:

Dose commitment period:

Dose units:

\begin{tabular}{lc} 
Radio- & $\begin{array}{c}\text { Inhalation } \\
\text { Effective } \\
\text { Dose } \\
\text { nuclide }\end{array}$ \\
\hline Equivalent \\
\hline PU 240 & $5.5 E+03$ \\
PU 238 & $1.5 E+03$ \\
PU 242 & $5.0 E-01$ \\
PU 241 & $2.8 E+03$ \\
U 237 & $0.0 E+00$ \\
AM 241 & $1.3 E+04$ \\
PU 239 & $4.3 E+03$ \\
\hline
\end{tabular}

Ingestion

Effective

Dose

Equivalent

--C-D---

$0.0 \mathrm{E}+00$

$0.0 \mathrm{E}+00$

$0.0 \mathrm{E}+00$

$0.0 \mathrm{E}+00$

$0.0 \mathrm{E}+00$

$0.0 \mathrm{E}+00$

$0.0 \mathrm{E}+00$
1.0

50.0

Rem

External

Dose

$1.6 \mathrm{E}-06$

5.1E-07

$0.0 \mathrm{E}+00$

1. $3 \mathrm{E}-10$

$0.0 \mathrm{E}+00$

1.9E-04

$9.4 \mathrm{E}-07$
Internal

Effective Dose Equivalent Equivalent

\section{$5.5 \mathrm{E}+03$}

1. $5 \mathrm{E}+03$

5. $0 \mathrm{E}-01$

$2.8 \mathrm{E}+03$

$0.0 \mathrm{E}+00$

1. $3 E+04$

4. $3 \mathrm{E}+03$
Annual

Effective Dose

5. $5 E+03$

1. $5 \mathrm{E}+03$

5. $0 \mathrm{E}-01$

2. $8 \mathrm{E}+03$

$0.0 \mathrm{E}+00$

1. $3 \mathrm{E}+04$

4. $3 \mathrm{E}+03$ 
Document Reviewed: C. H. Huang, "X/Qs AND UNIT DOSE CALCULATIONS FOR CENTRAL WASTE COMPLEX INTERIM SAFETY BASIS EFFORT,"

March 13, 1996.

Scope of Review: Entire Document

Yes No NA

[ ] [ ] BA* Previous reviews complete and cover analysis, up to scope of this review, with no gaps.

Problem completely defined.

Accident scenarios developed in a clear and logical manner.

Necessary assumptions explicitly stated and supported.

Computer codes and data files documented.

Data used in calculations explicitly stated in document.

Data checked for consistency with original source information as applicable.

[ ] [ ] A Mathematical derivations checked including dimensional consistency of results.

$\triangle$ [ ] [ ] Models appropriate and used within range of validity or use outside range of established validity justified.

4 [ ] [ ] Hand calculations checked for errors. Spreadsheet results should be treated exactly the same as hand calculations. Software input correct and consistent with document reviewed. Software output consistent with input and with results reported in document reviewed. Limits/criteria/guidelines applied to analysis results are appropriate and referenced. Limits/criteria/guidelines checked against references. Safety margins consistent with good engineering practices. Conclusions consistent with analytical results and applicable limits.

LA [ ] [ ] Results and conclusions address all points required in the problem statement.

[ ] [ ] $[$ Format consistent with appropriate NRC Regulatory Guide or other standards

[ ] [ $] \star$ Review calculations, comments, and/or notes are attached.

$凶[$ ] [ ]

Document approved.

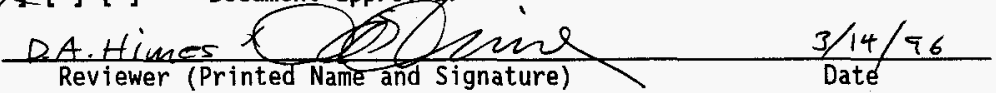




\section{HEDOP REVIEW CHECKLIST}

for

Radiological and Nonradiological Release Calculations

Document Reviewed: $C$. H. Huang, "X/QS AND UNIT DOSE CALCULATIONS FOR CENTRAL WASTE COMPLEX INTERIM SAFETY BASIS EFFORT,"

March 13, 1996

Submitted by: C. H. HUANG

Date Submitted: March 12, 1996

Scope of Review: Entire Document

YES NO* N/A

Q4 [ ] [ ] 1. A detailed technical review and approval of the environmental transport and dose calculation portion of the analysis has been performed and documented.

[ ] [ ] D4 2. Detailed technical review(s) and approval(s) of scenario and release determinations have been performed and documented.

4A [ ] [ ] 3. HEDOP-approved code(s) were used.

LA [ ] [ 4. Receptor locations were selected according to HEDOP recommendations.

14 [ ] [ ] 5. All applicable environmental pathways and code options

[4 [ ] [ ] 6. Hanford site data were used. justified and performed correctly.

[] [ ] [ ]

8. The analysis is consistent with HEDOP recommendations.

9. Supporting notes, calculations, comments, comment resolutions, or other information is attached. (Use the "Page 1 of $X "$ page numbering format and sign and date each added page.)

D [ ] 10. Approval is granted on behalf of the Hanford Environmental Dose Overview Panel.

* A11 "NO" responses must be explained and use of nonstandard methods justified.

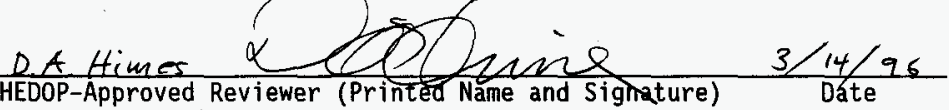

COMMENTS (add additional signed and dated pages if necessary): 


\section{DISTRIBUTION SHEET}

\begin{tabular}{|c|c|c|c|c|c|}
\hline \multirow{2}{*}{$\begin{array}{l}\text { To } \\
\text { D.J. Schmitt }\end{array}$} & \multirow{2}{*}{\multicolumn{3}{|c|}{$\begin{array}{l}\text { From } \\
\text { C.H. Huang }\end{array}$}} & \multicolumn{2}{|l|}{ Page 1 of 1} \\
\hline & & & & \multicolumn{2}{|l|}{ Date $3 / 12 / 96$} \\
\hline \multicolumn{4}{|l|}{ Project Title/Work Order } & \multicolumn{2}{|c|}{ EDT No. 156464} \\
\hline \multicolumn{4}{|c|}{$\begin{array}{l}X / Q S \text { AND UNIT DOSE CALCULATIONS FOR CENTRAL WASTE COMPLEX } \\
\text { INTERIM SAFETY BASIS EFFORT }\end{array}$} & \multicolumn{2}{|l|}{ ECN No. N/A } \\
\hline Name & MSIN & $\begin{array}{c}\text { Text } \\
\text { With All } \\
\text { Attach. }\end{array}$ & Text Only & $\begin{array}{l}\text { Attach./ } \\
\text { Appendix } \\
\text { Only }\end{array}$ & $\begin{array}{l}\text { EDT/ECN } \\
\text { Only }\end{array}$ \\
\hline $\begin{array}{l}\text { D.J. Schmitt } \\
\text { D.A. Himes } \\
\text { J.C. Van Keuren }\end{array}$ & $\begin{array}{l}\text { T3-01 } \\
\text { A3-34 } \\
\text { A3-34 } \\
\end{array}$ & $\begin{array}{l}x \\
X \\
X\end{array}$ & & & \\
\hline & 22 & $V$ & & & \\
\hline
\end{tabular}

